\title{
Medical gallery of Blausen Medical 2014
}

Correspondence: info@blausen.com

\section{Abstract}

This is a gallery of images contributed to Wikimedia projects by Blausen Medical by 2014 .

\section{Contents}

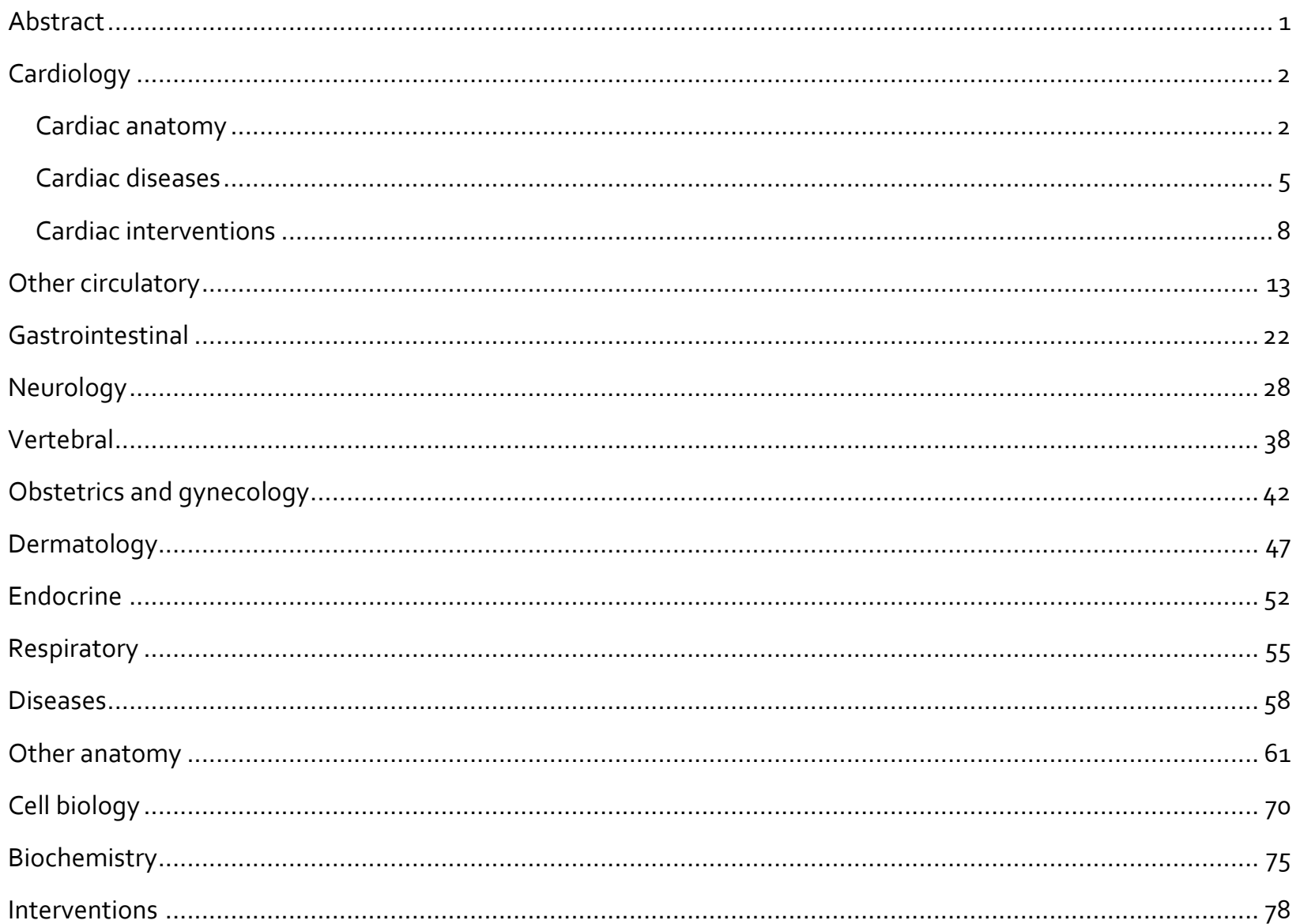




\section{Cardiology}

\section{Cardiac anatomy}
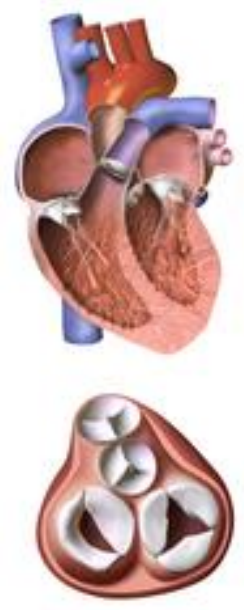

Heart valves.

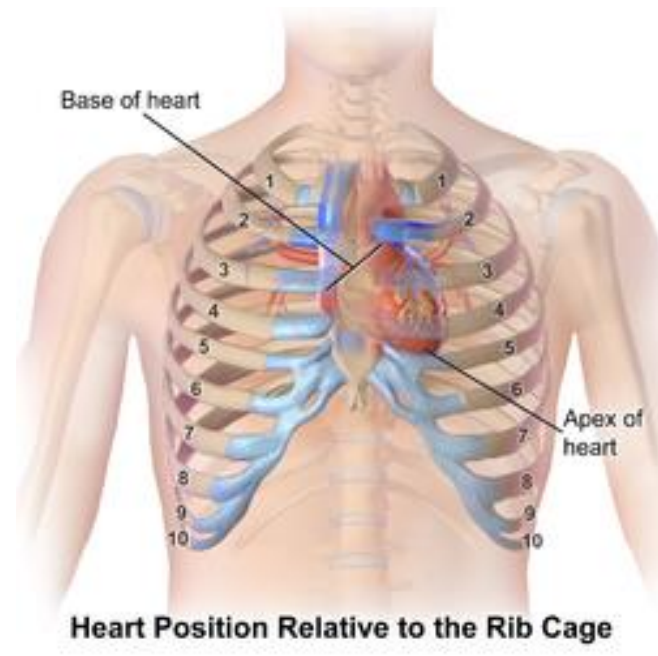

Heart location

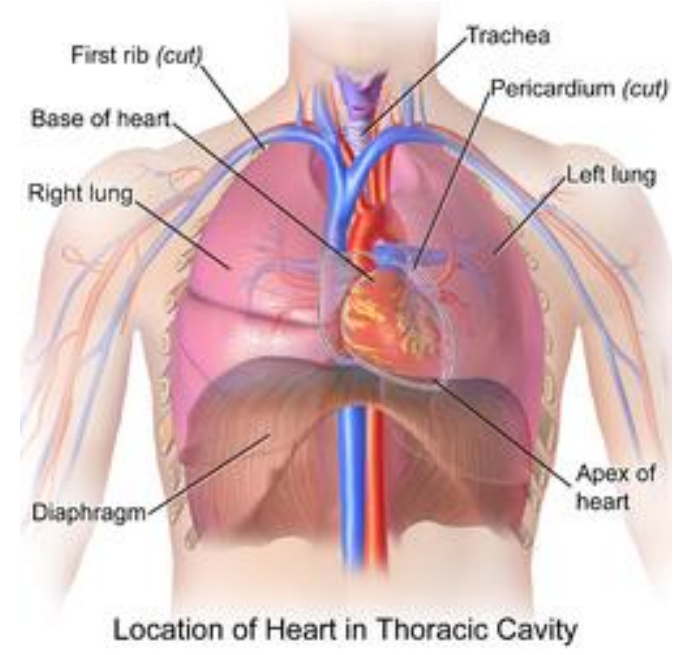

Thoracic cavity.

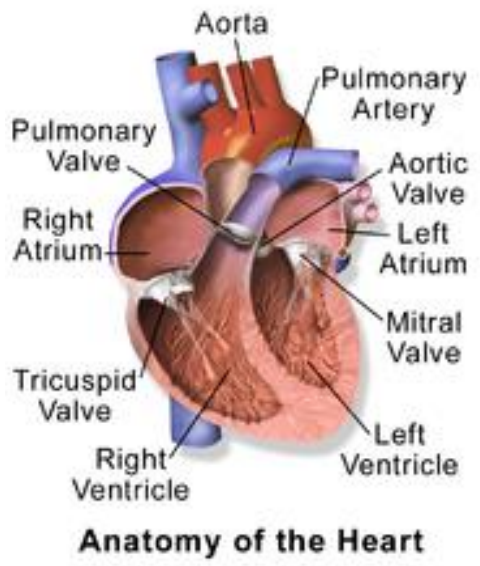

Heart anatomy 


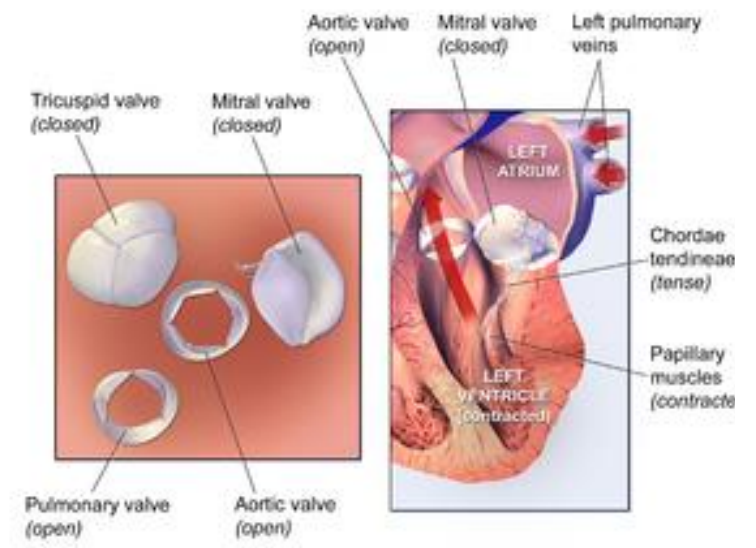

Valves of the Heart (Ventricles Contracting)

Heart valves during contraction of the ventricles.

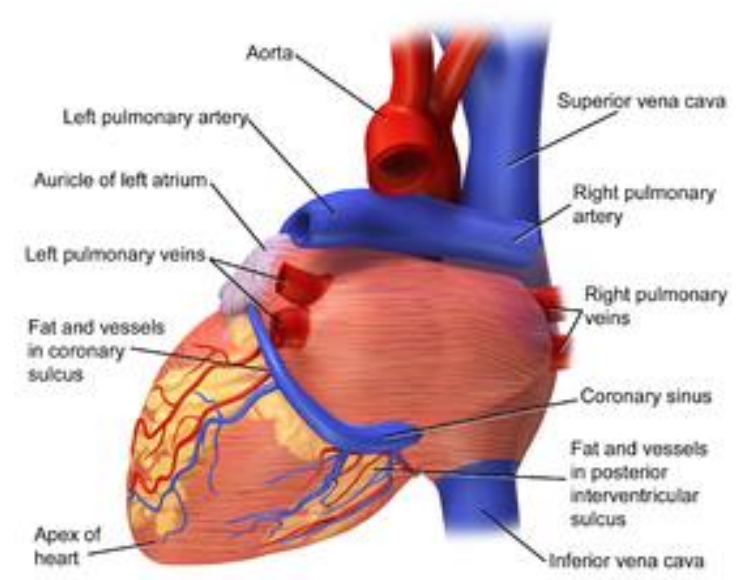

Superficial Heart Anatomy (Posterior)

Posterior view of the heart

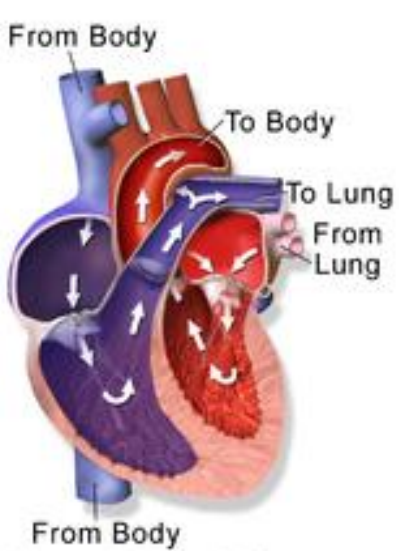

Normal Blood Flow

Circulatory system through the heart.

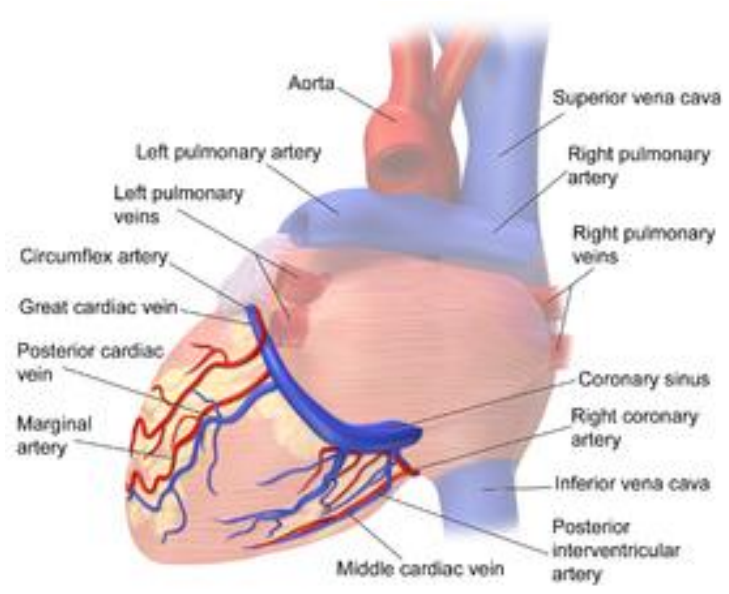

Coronary Circulation (Posterior)

Posterior view of coronary circulation. 


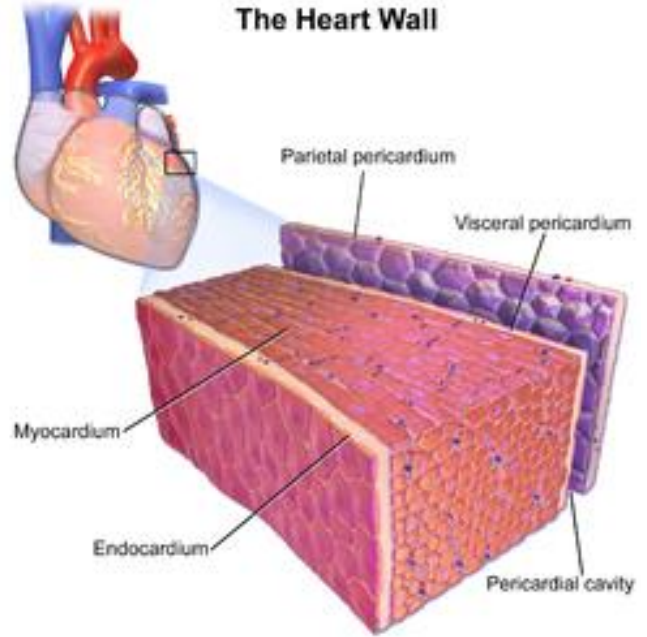

Layers of the walls of the heart. 


\section{Cardiac diseases}

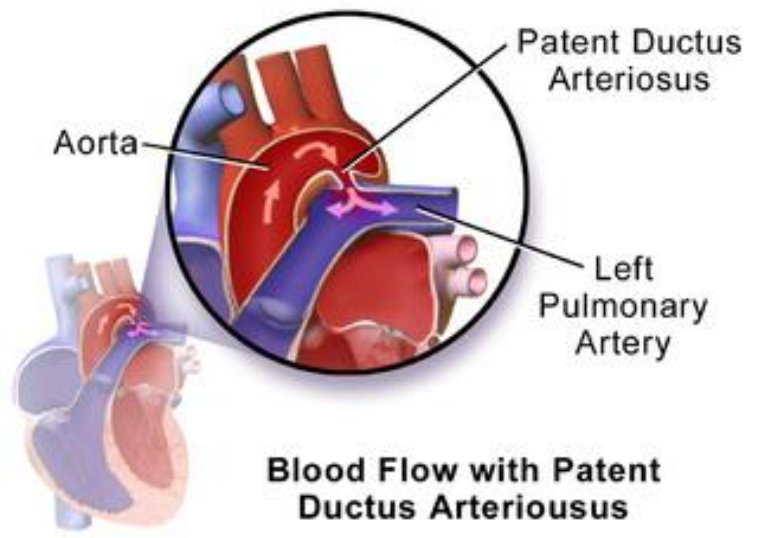

Patent ductus arteriosus.

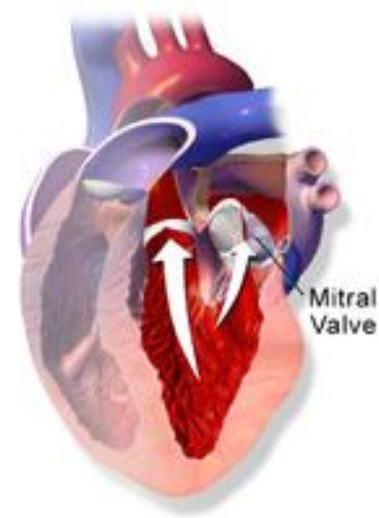

Mitral Valve Regurgitation

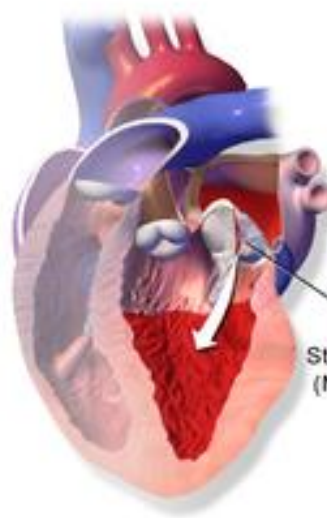

Mitral Valve Stenosi
Mitral valve regurgitation and stenosis.

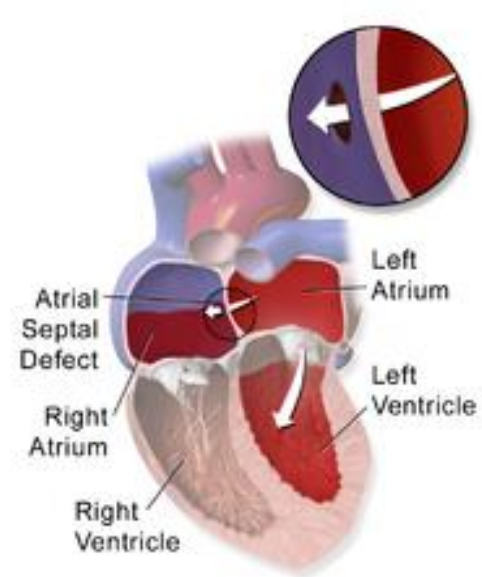

Heart with Atrial Septal Defect

Atrial septal defect.

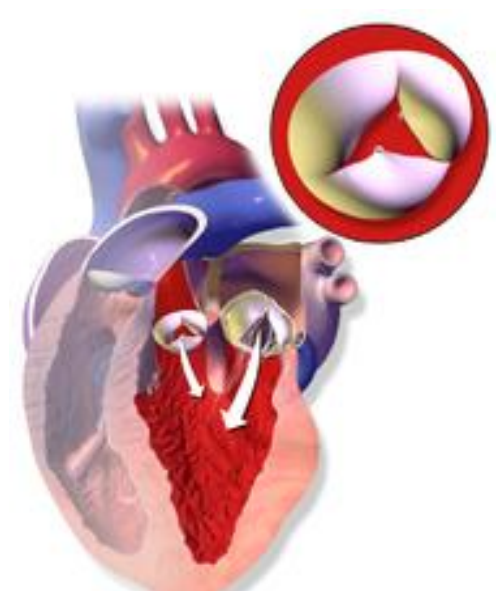

Aortic Regurgitation

Aortic valve regurgitation 
우

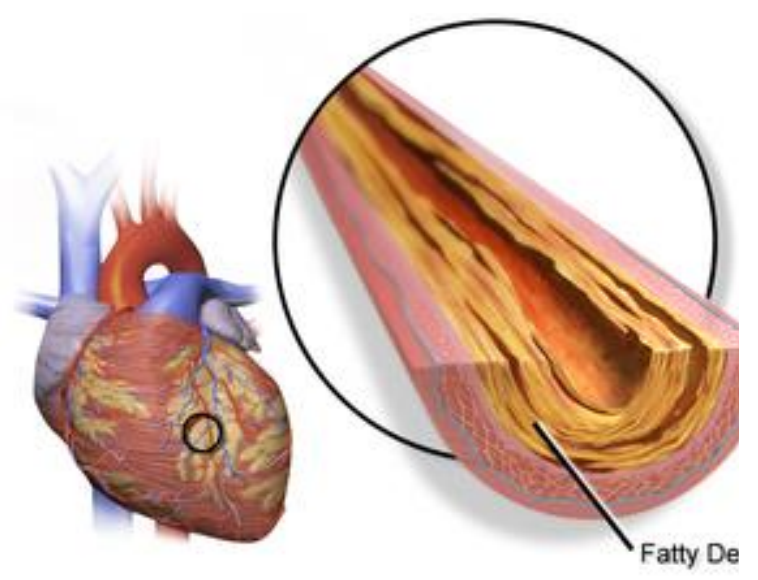

Atherosclerotic plaque of a coronary artery.

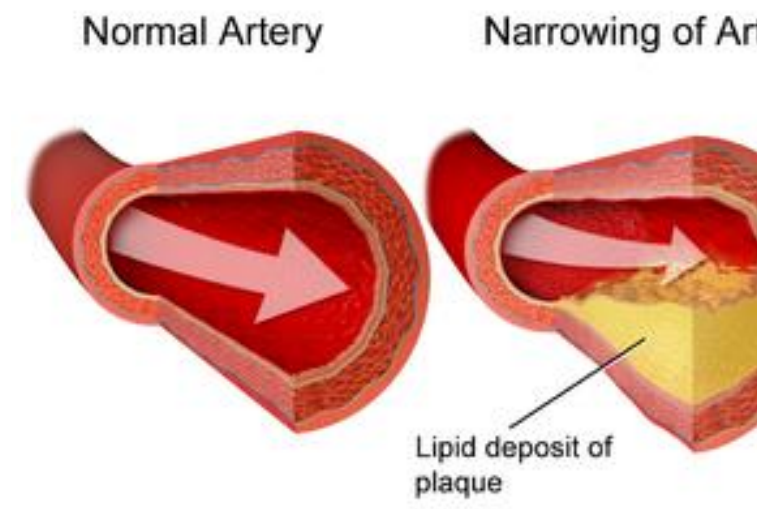

Coronary Artery Disease

Coronary artery disease.
WikiJournal of Medicine, 2014, 1(2) doi: $10.15347 / \mathrm{wjm} / 2014.010$

Figure Article

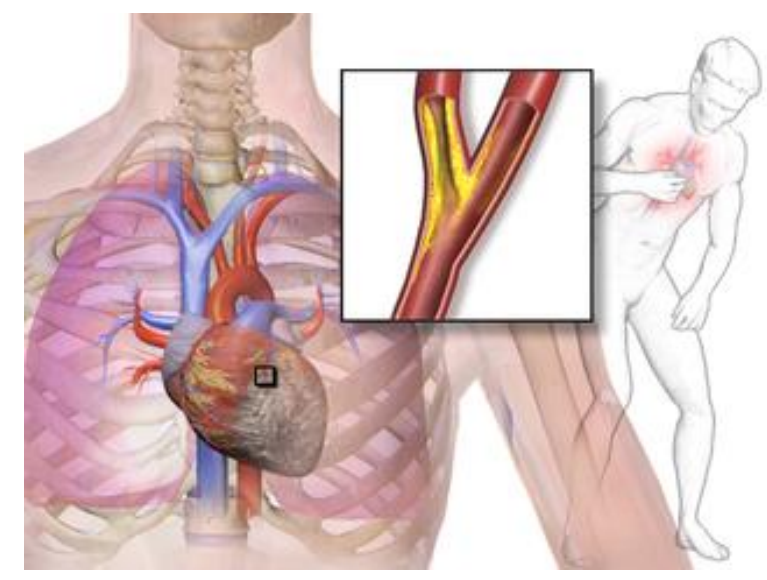

Angina pectoris.

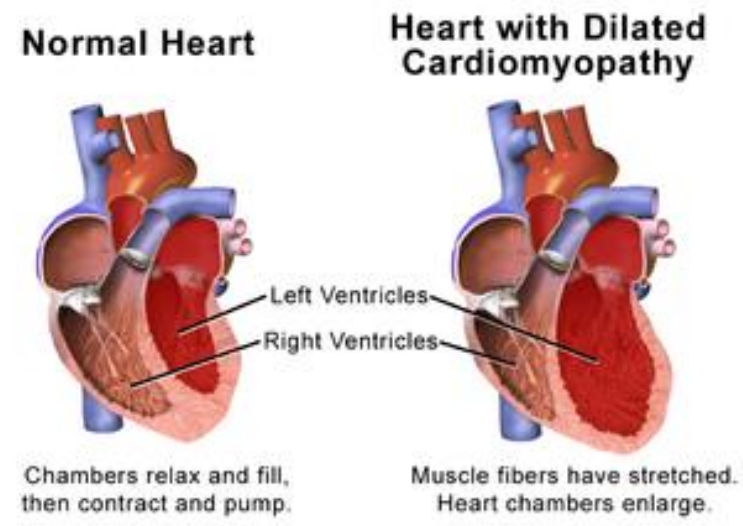

Normal heart versus one with dilated cardiomyopathy 


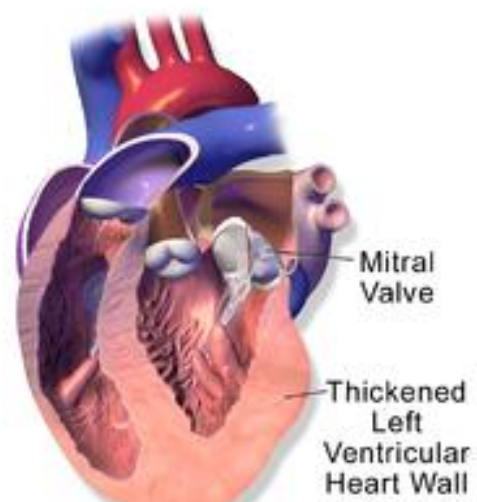

Hypertrophic Cardiomyopathy

Hypertrophic cardiomyopathy.

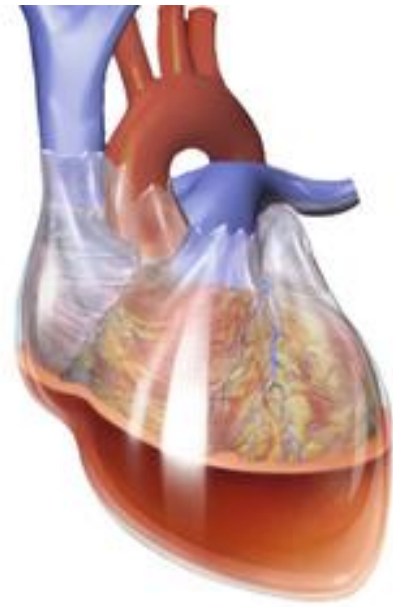

Hemopericardium

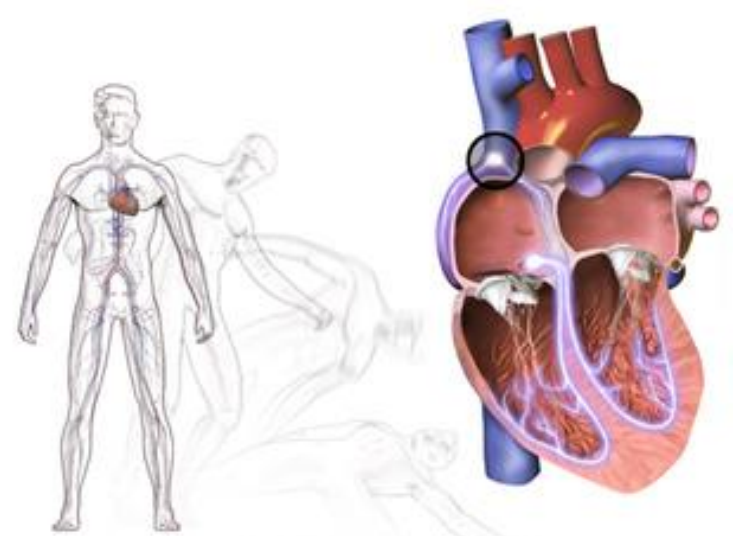

Fainting from bradycardia.

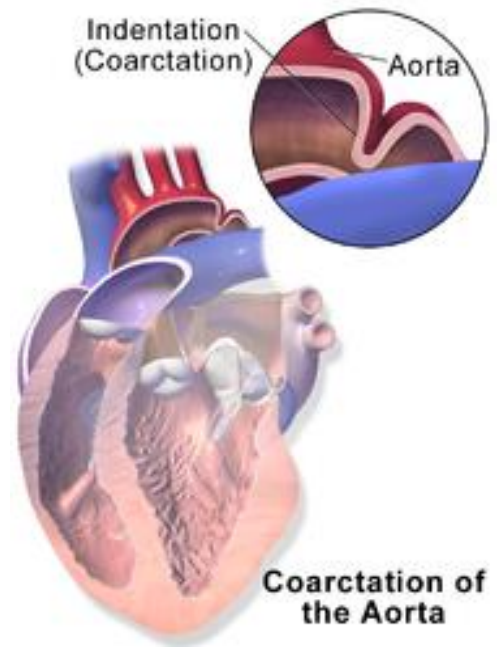

Coarctation of the aorta. 


\section{Cardiac interventions}

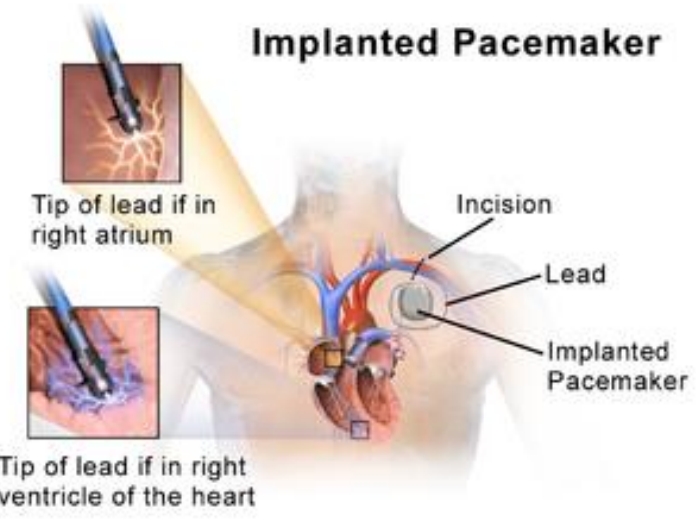

Placement of a pacemaker.

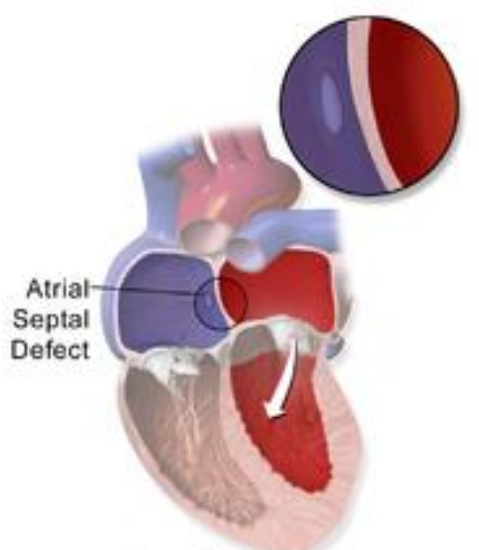

Patch Closure for Atrial Septal Defect

Correction of an atrial septal defect using a patch.

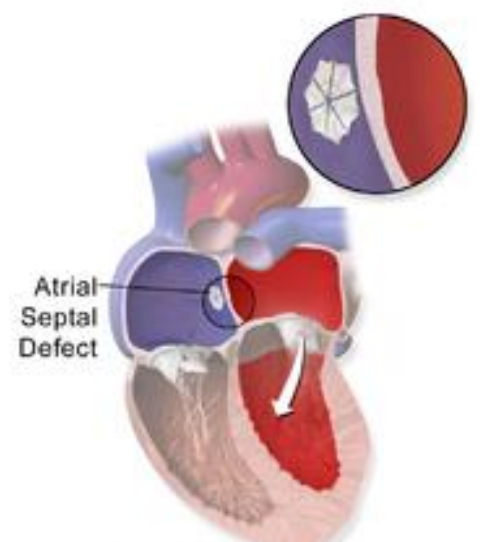

Device Closure for Atrial Septal Defect

Correction of an atrial septal defect using a percutaneous device.

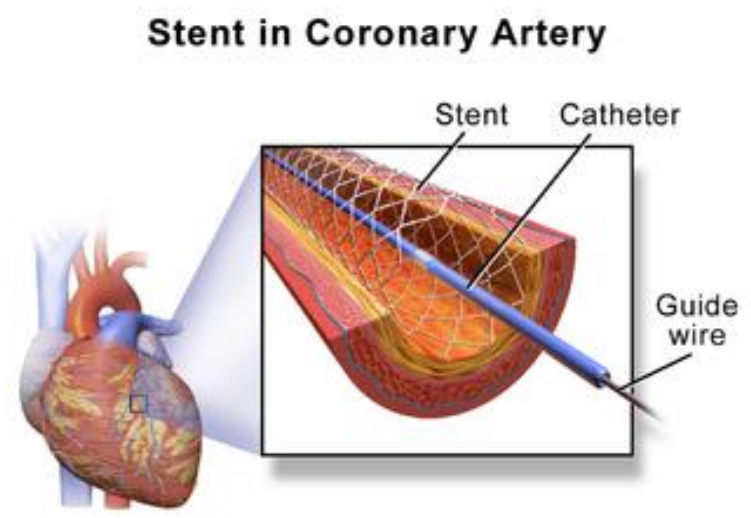

A coronary stent placed by percutaneous coronary intervention. 


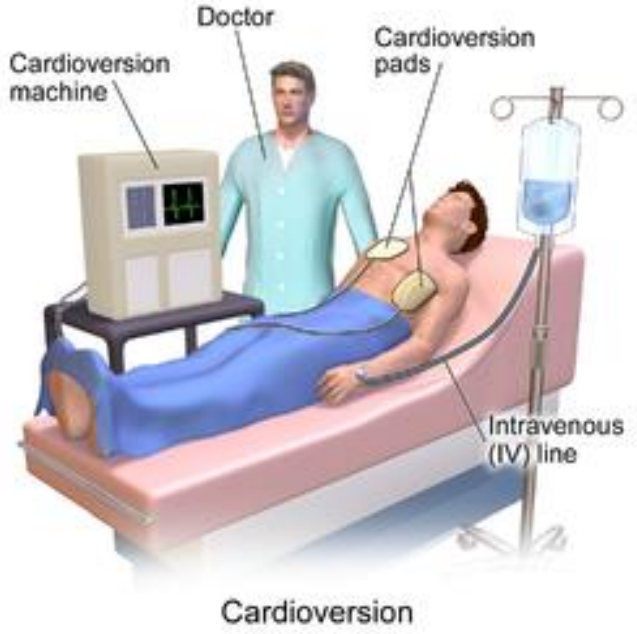

The setting of cardioversion

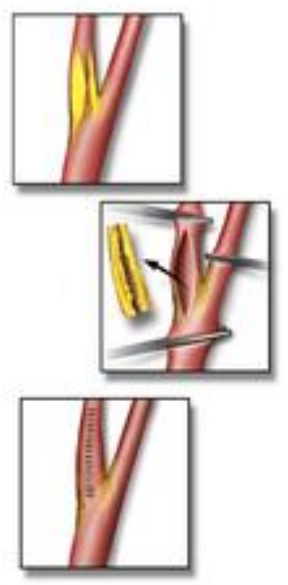

Endarterectomy.

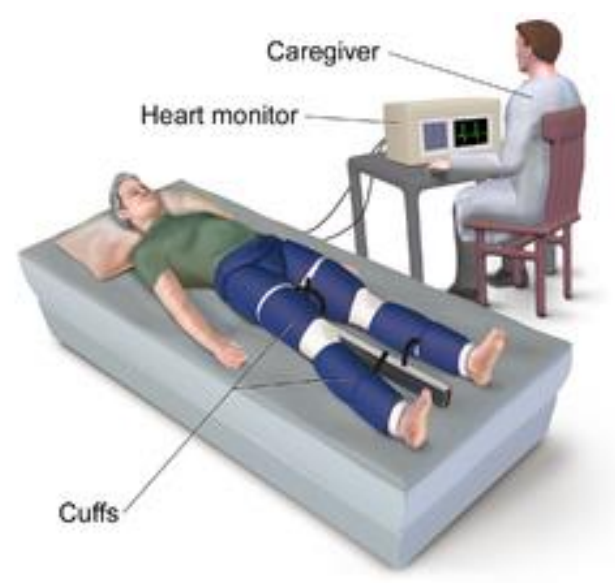

Cardiac Enhanced External Counterpulsation

Enhanced external counterpulsation.

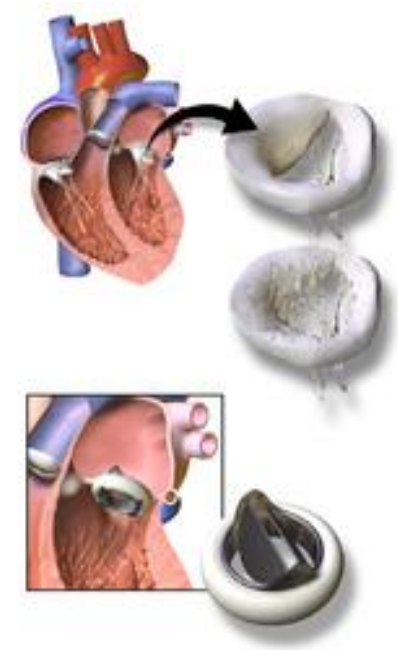

Artificial heart valve of St. Francis model 

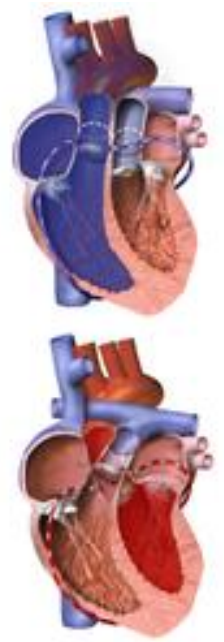

Arterial switch procedure to correct dextroTransposition of the great arteries.
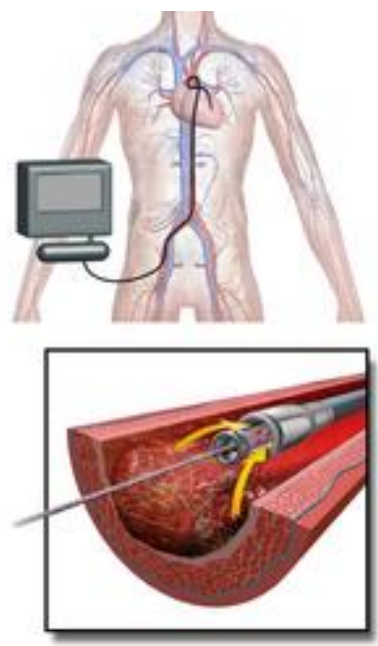

Coronary thrombectomy by AngioJet technique.

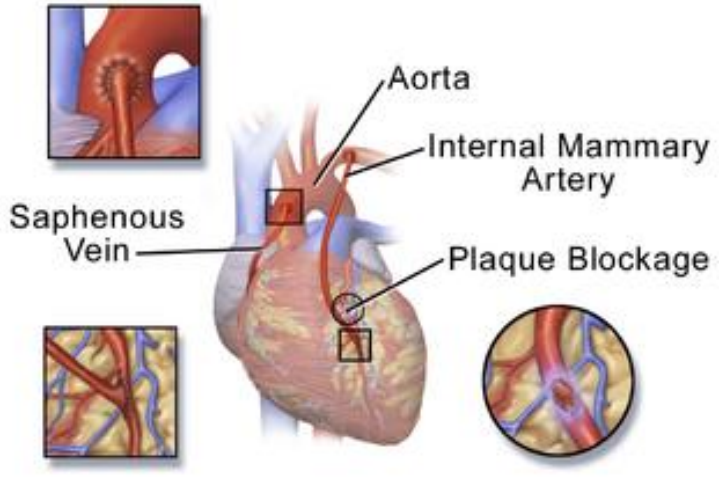

\section{Coronary Artery Bypass Surgery}

Coronary artery bypass surgery.

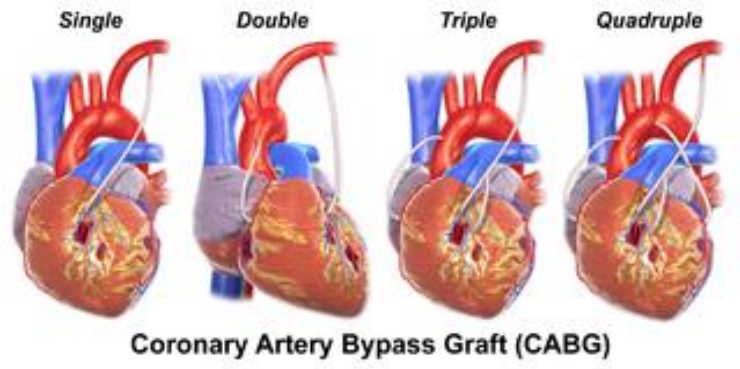

Coronary artery bypass surgery types.

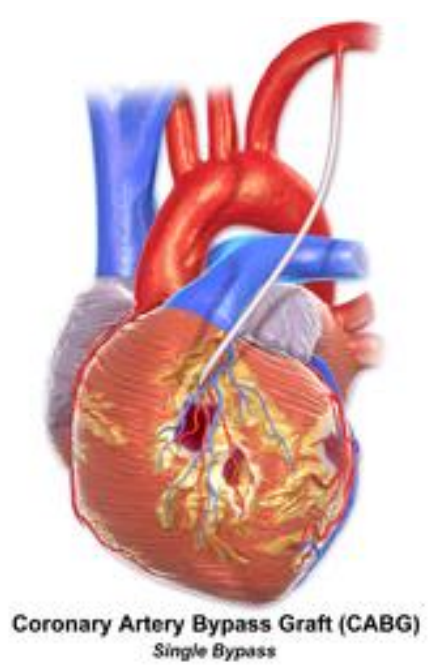

Single coronary artery bypass. 


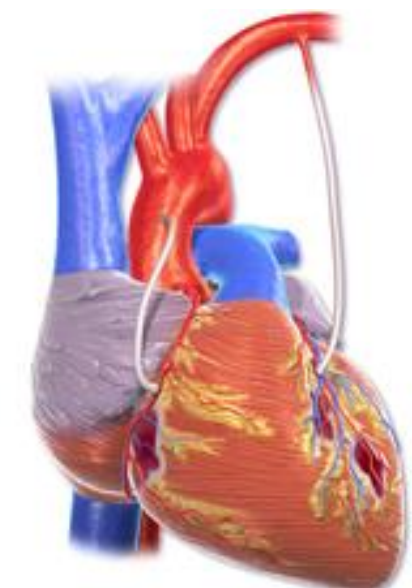

Coronary Artery Bypass Graft (CABG) Double Bypess

Double coronary artery bypass.

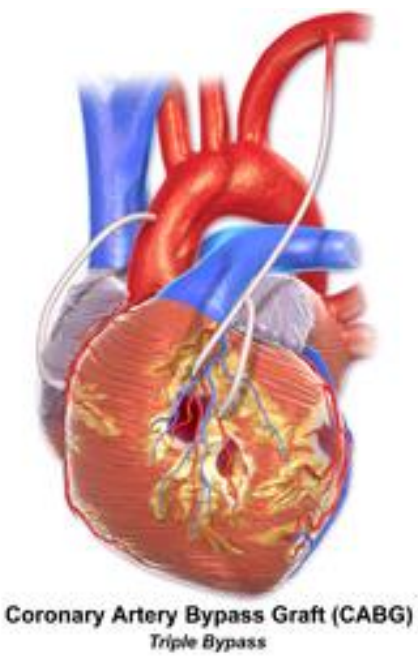

Triple coronary artery bypass.

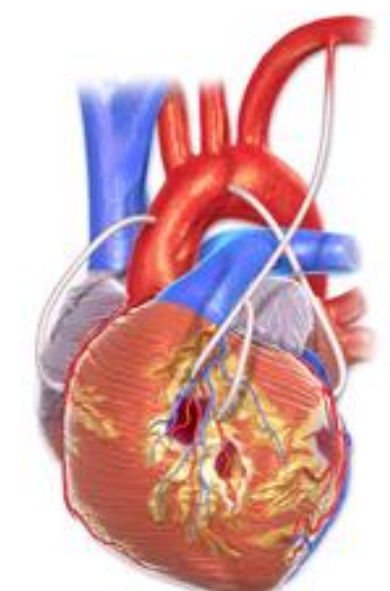

Coronary Artery Bypass Graft (CABG) Quadruple Bypass

Quadruple coronary artery bypass.

\section{Implantable Cardioverter Defibrillator}

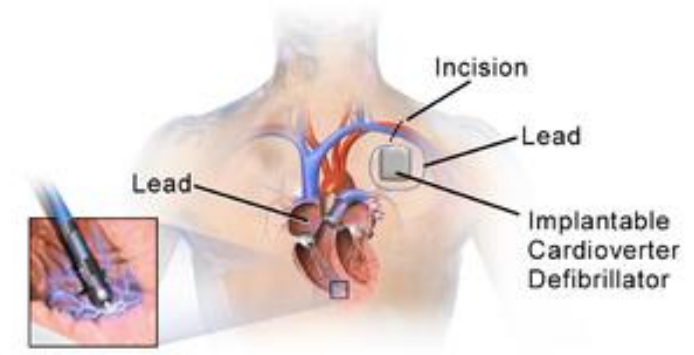

Tip of lead in right ventricle of Heart

Implantable cardioverter-defibrillator. 
우

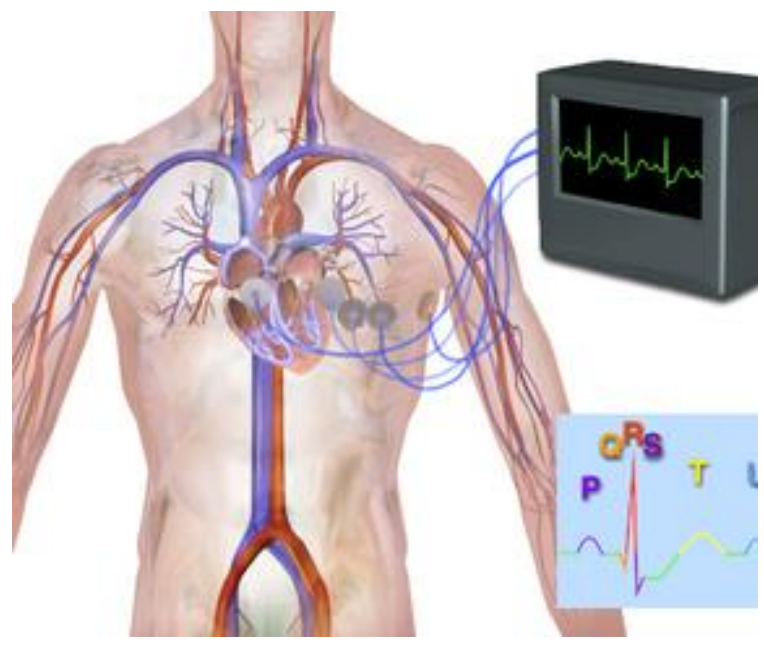

WikiJournal of Medicine, 2014, 1(2) doi: $10.15347 / \mathrm{wjm} / 2014.010$ Figure Article

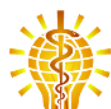

Electrocardiography. 


\section{Other circulatory}

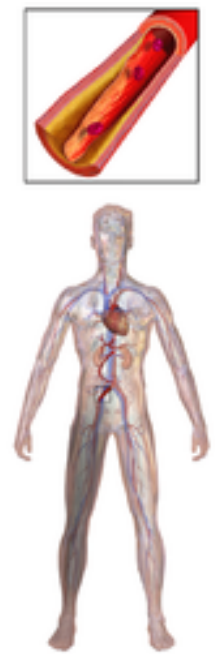

Peripheral artery disease.

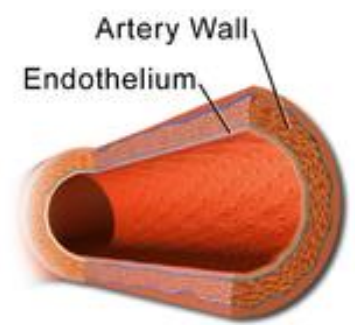

Normal Artery

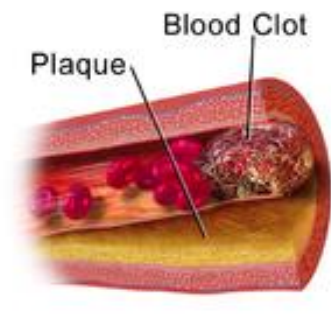

Diseased Artery
Normal artery vs. one

with atherosclerotic plaque and blood clot.

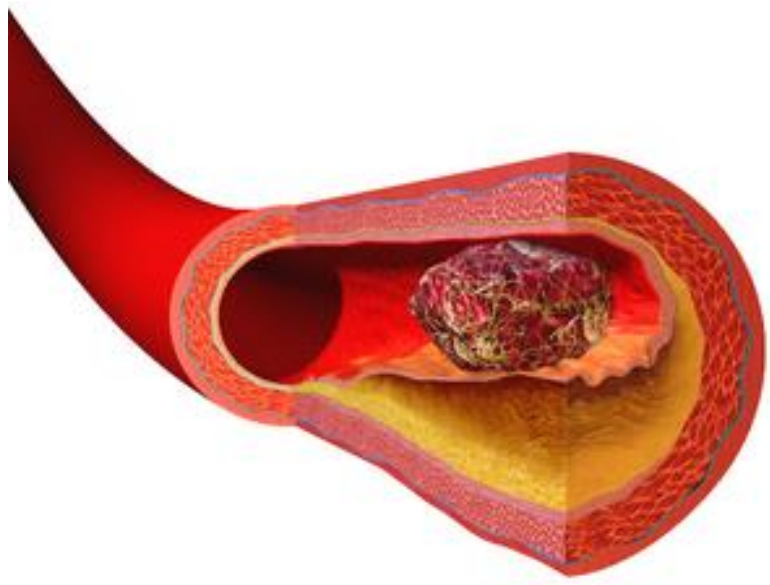

Blood clot.

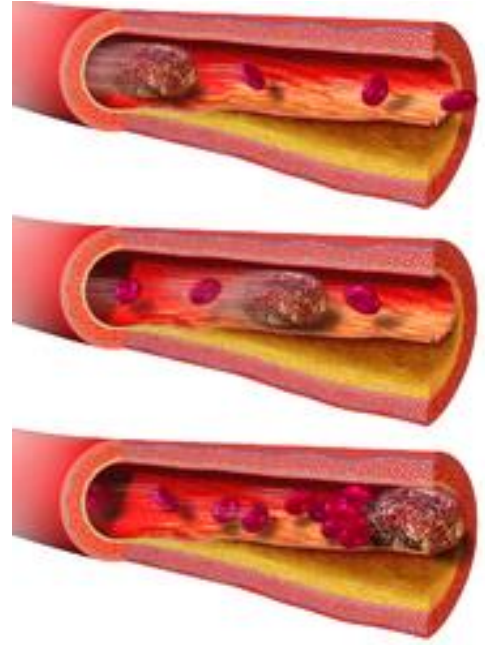

Blood clot motion. 


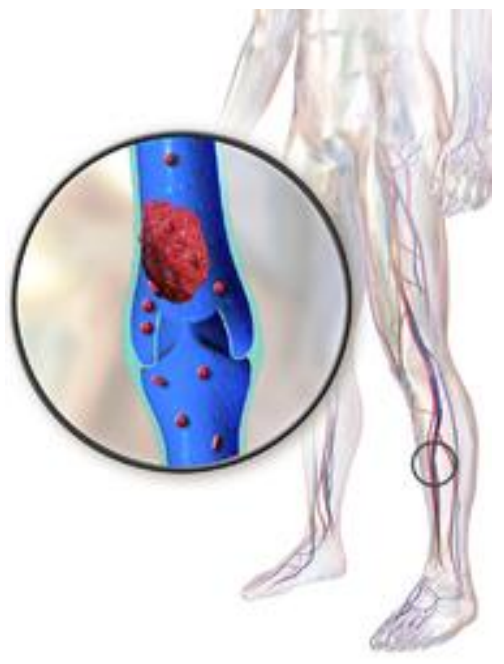

Deep vein thrombosis.

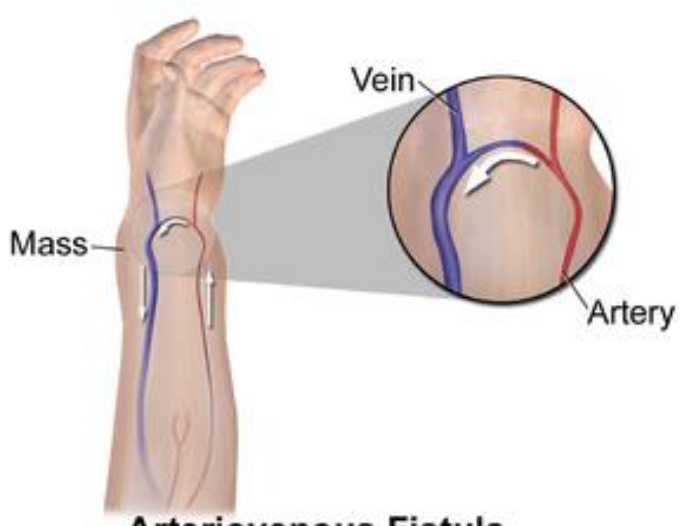

Arteriovenous Fistula

Arteriovenous fistula.

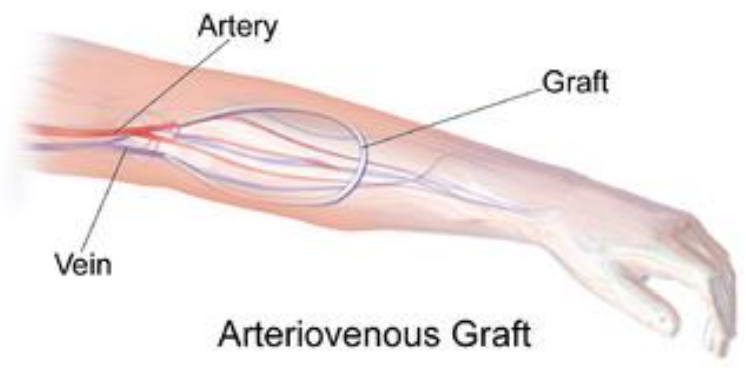

Arteriovenous graft for hemodialysis.

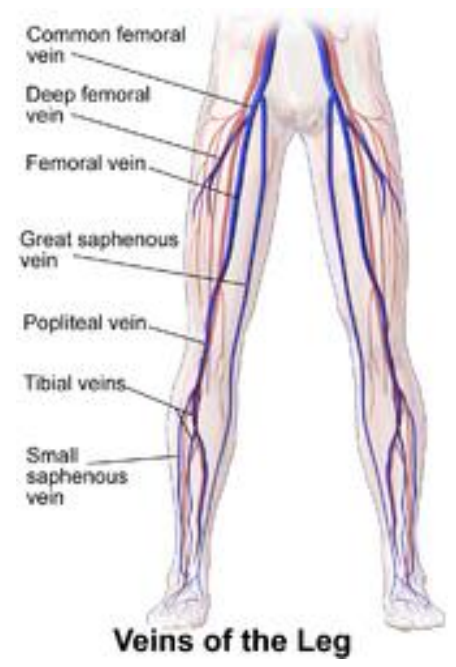

Veins of the leg.

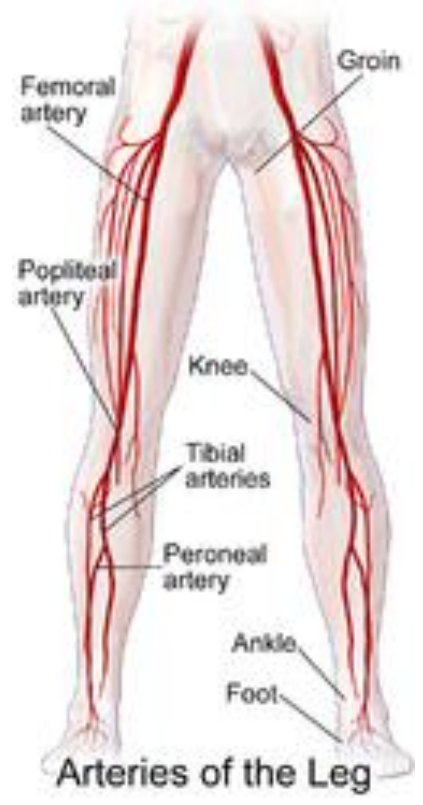

Arteries of the leg. 


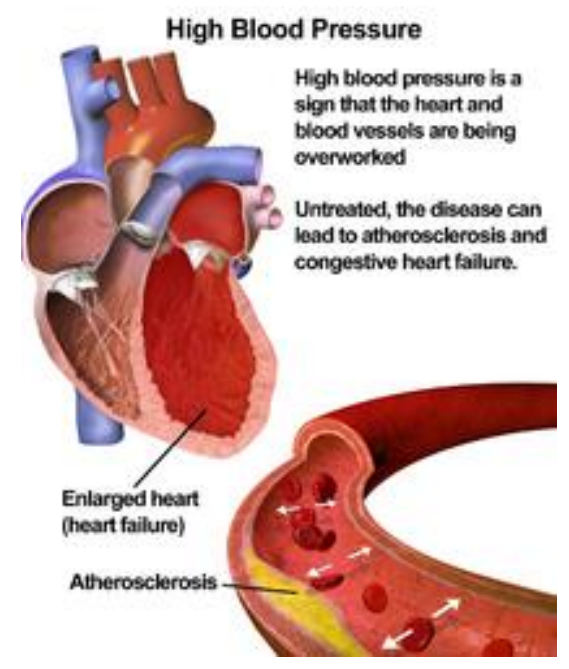

Hypertension.

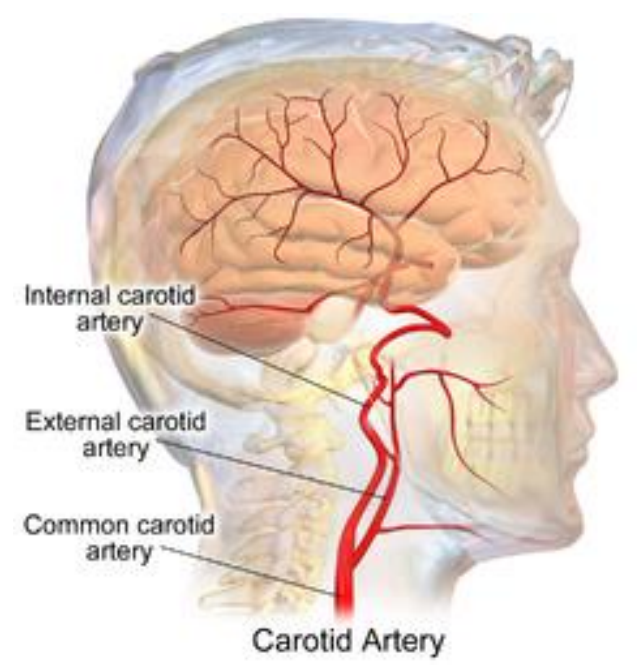

Carotid arteries.

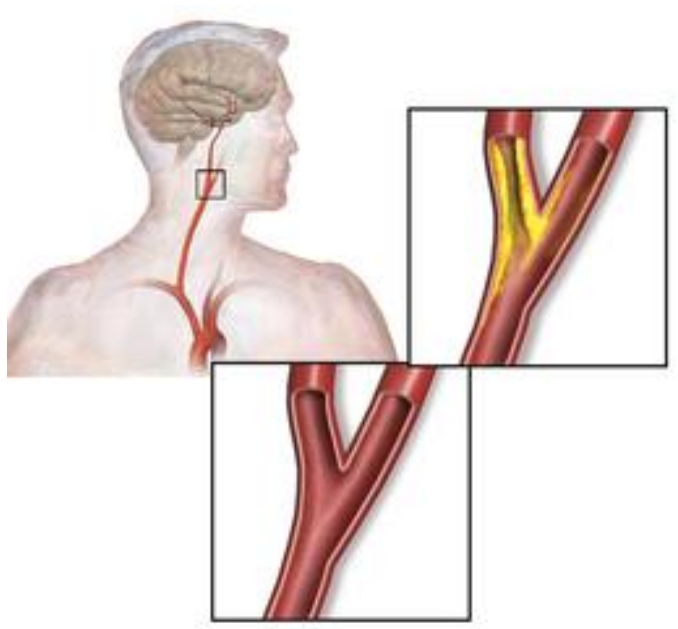

Carotid artery stenosis.

\section{The Structure of an Artery Wall}

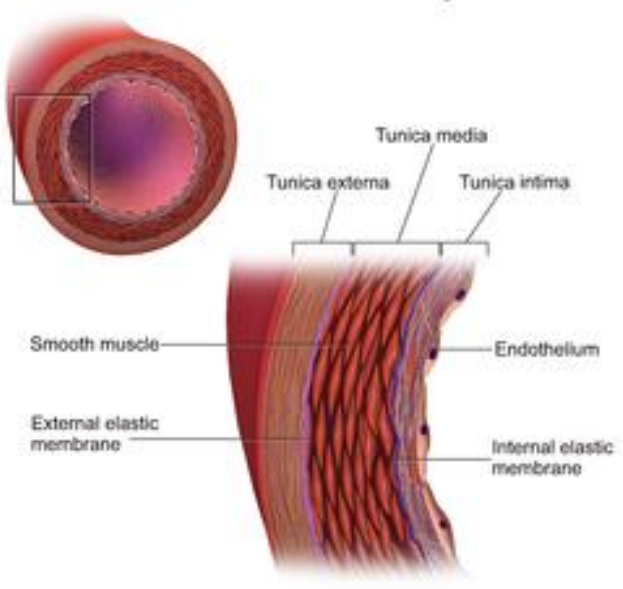

Arterial wall. 


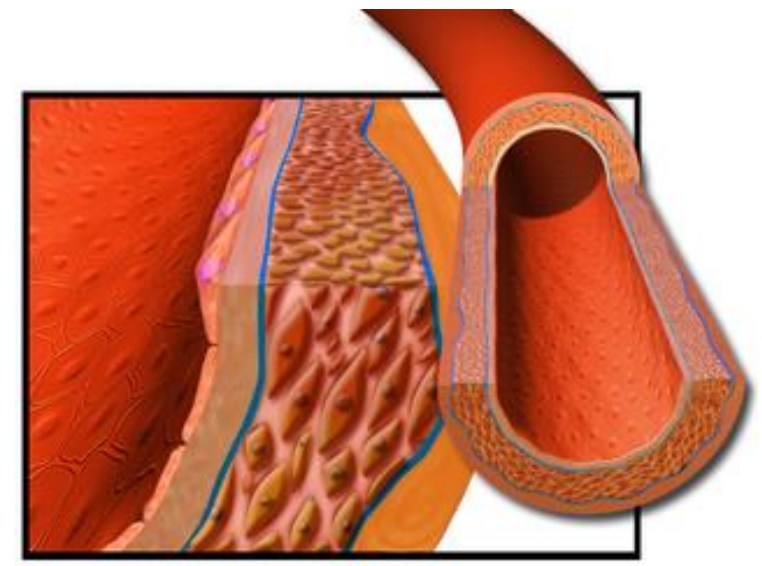

Artery.

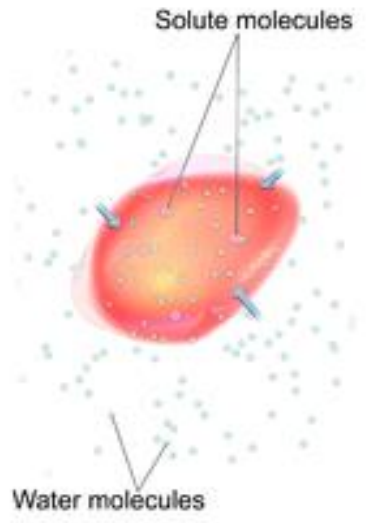

Hypotonic Solution (Osmotic Flow into Cell)

A red blood cell in a hypotonic solution.

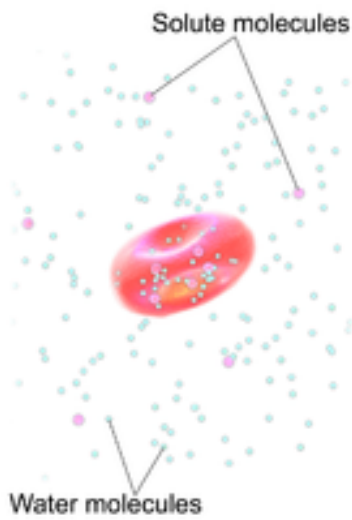

Isotonic Solution

(No Osmotic Flow)

A red blood cell in an isotonic solution.

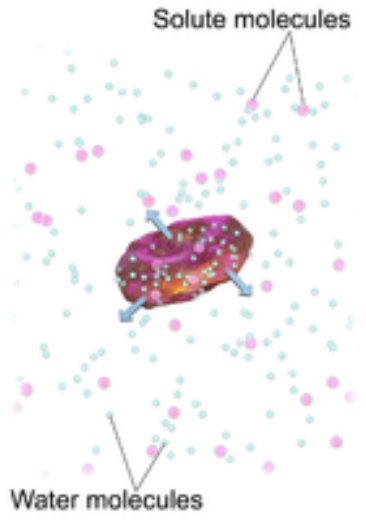

Hypertonic Solution (Osmotic Flow out of Cell)

A red blood cell in a hypertonic solution. 


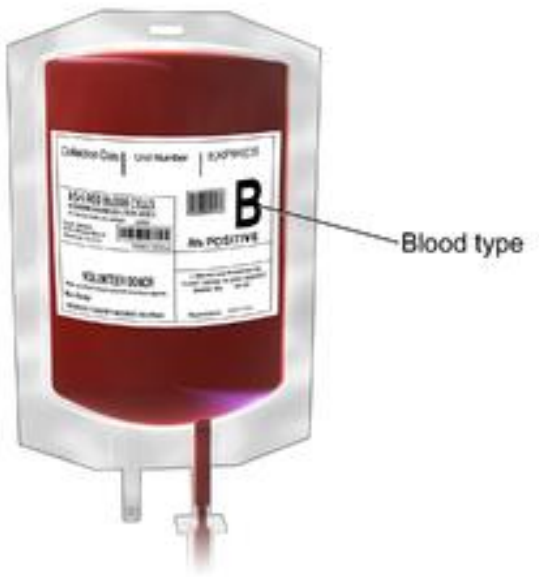

Labeled Blood Bag

Bag of packed red blood cells.

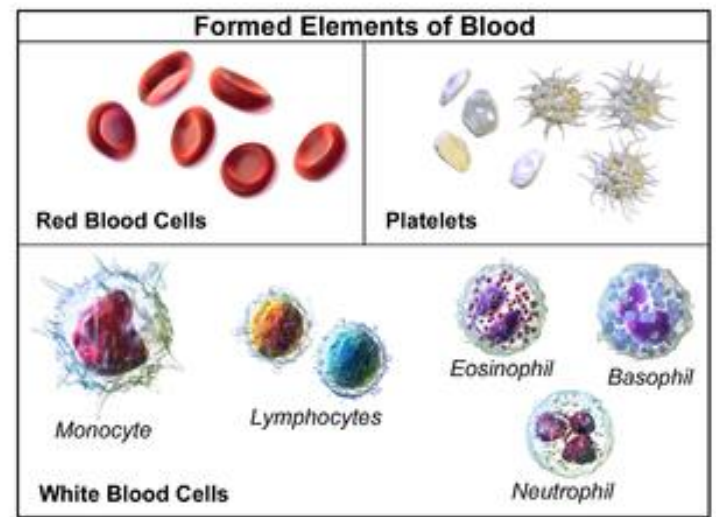

Cell types of blood.

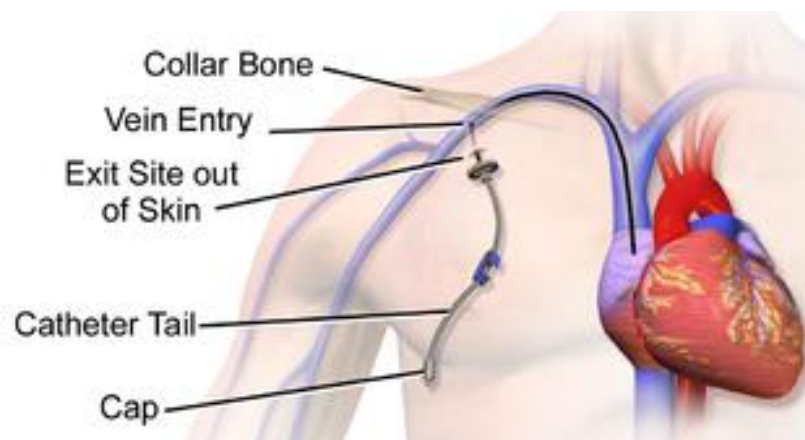

\section{Non-Tunneled Central Venous Access Device}

Non-tunneled central venous catheter.

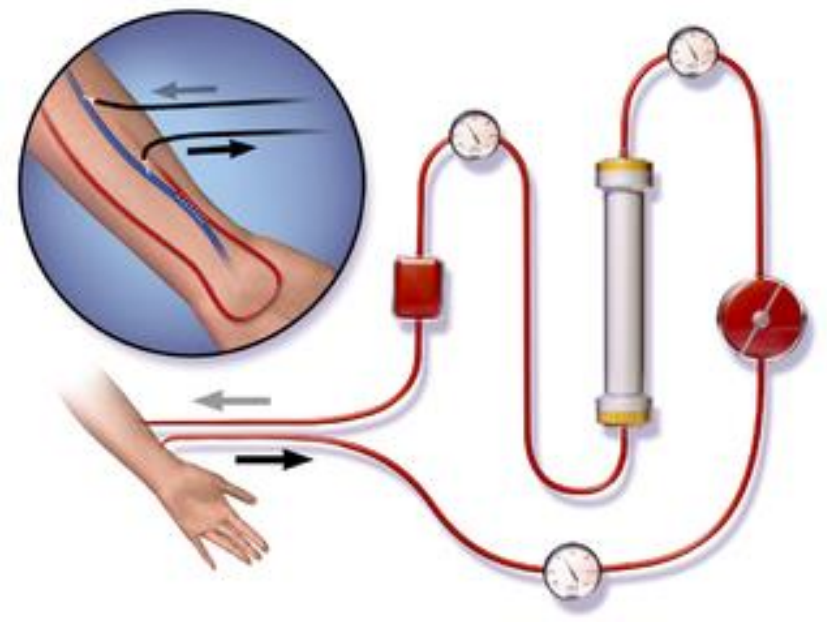

Hemodialysis. 


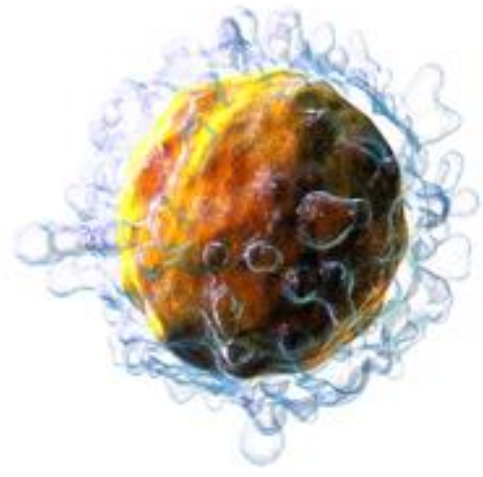

\section{Lymphocyte}

$T$ cell

T cell.

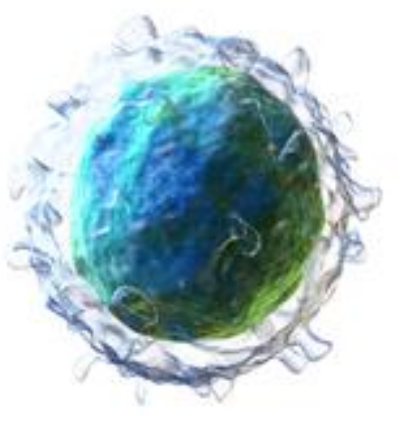

\section{Lymphocyte}

$B$ cell

B cell.

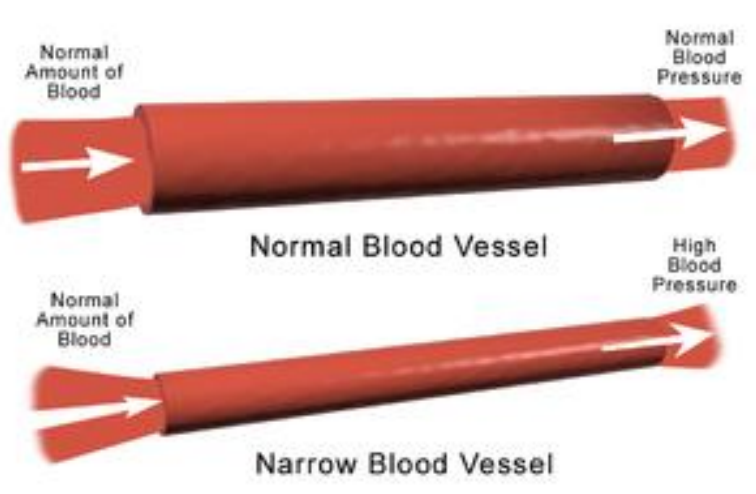

\section{Blood Pressure Blood Flow}

Blood pressure flow.

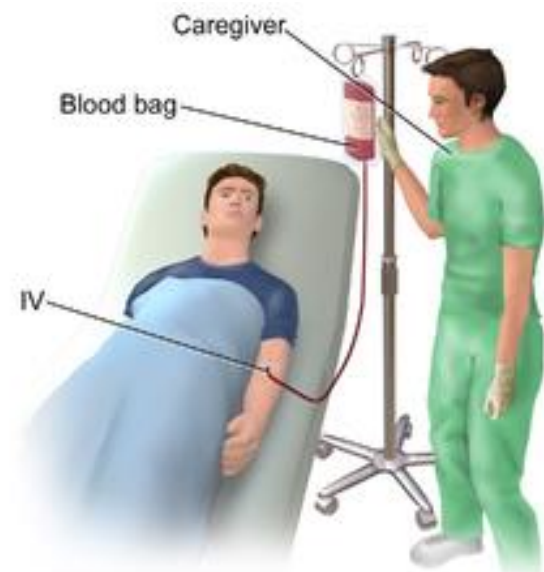

Blood Transfusion

Blood transfusion 


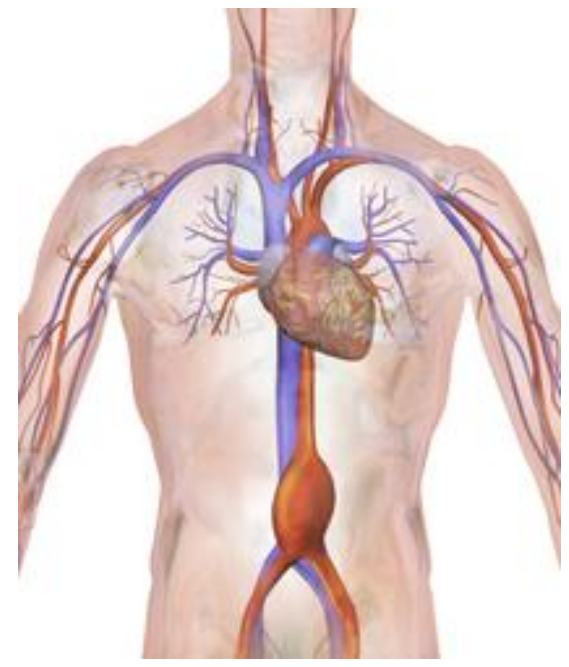

Abdominal aortic aneurysm.

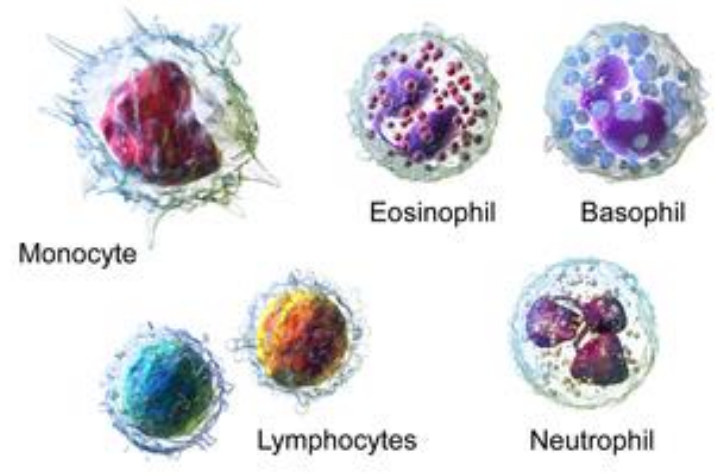

White Blood Cells

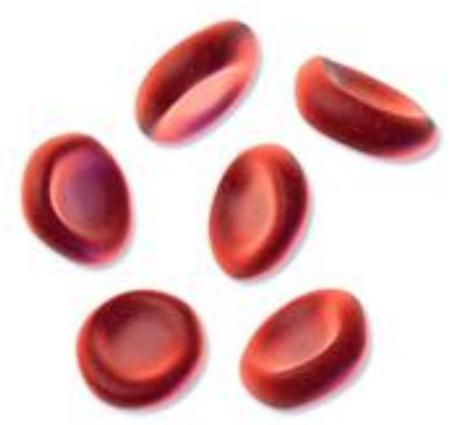

\section{Red Blood Cells}

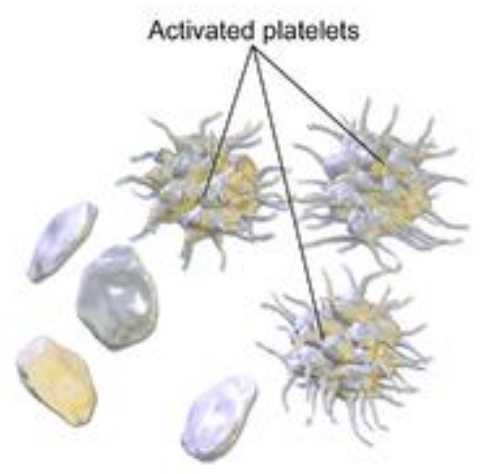

Platelets

White blood cells.

Platelets. 


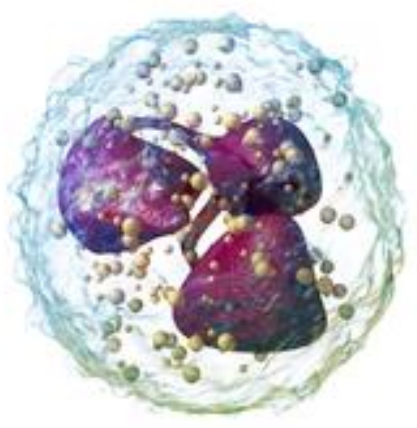

Neutrophil

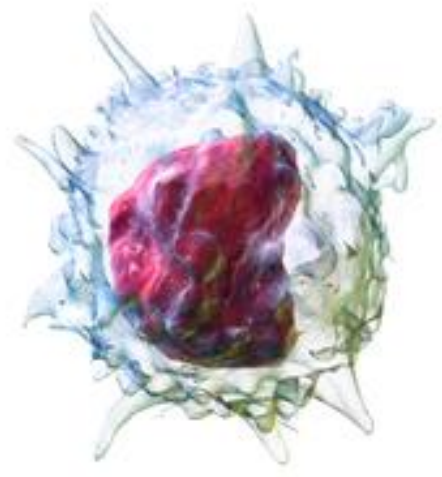

Monocyte

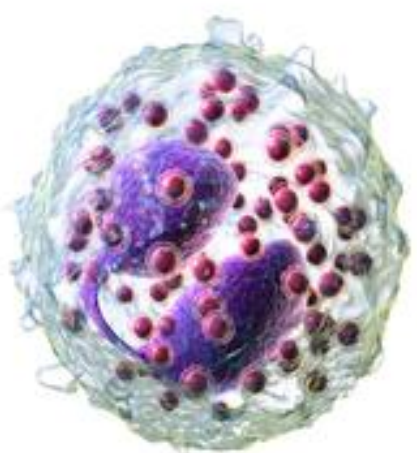

Eosinophil
Neutrophil granulocyte.

Monocyte.
Eosinophil granulocyte.

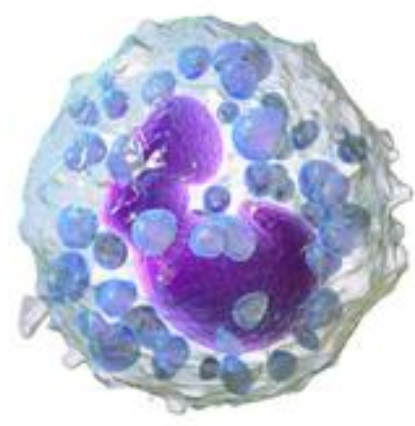

\section{Basophil}

Basophil granulocyte. 


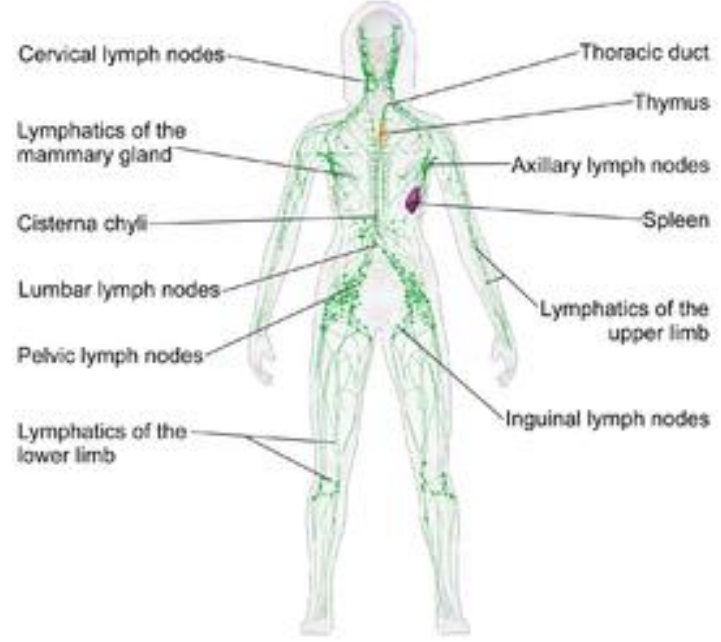

Lymphatic system. 


\section{Gastrointestinal}

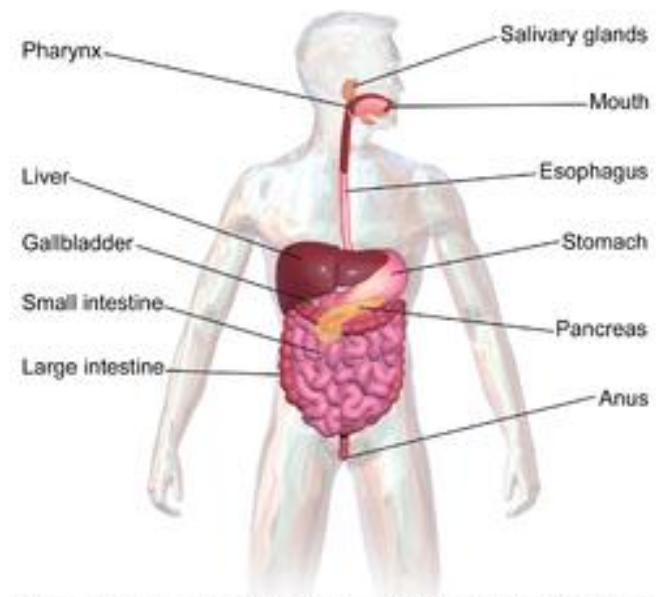

The Components of the Digestive System

Digestive system.

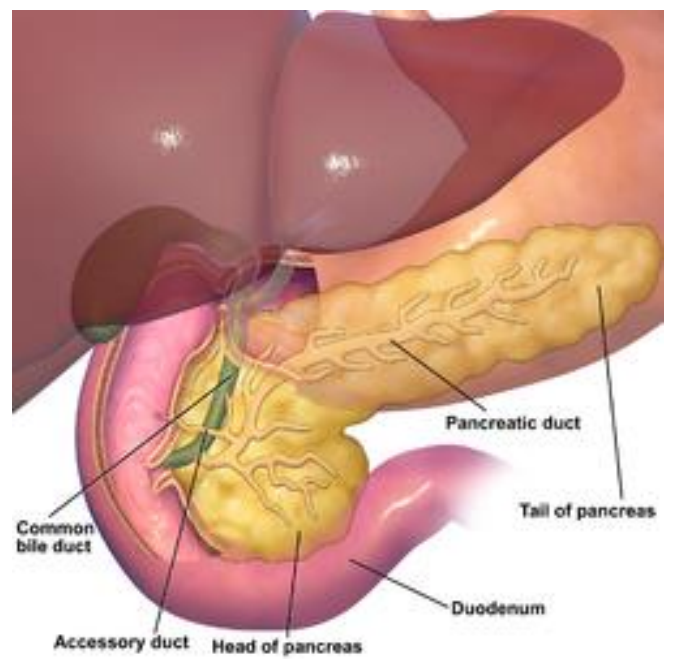

Anatomy of the pancreas.

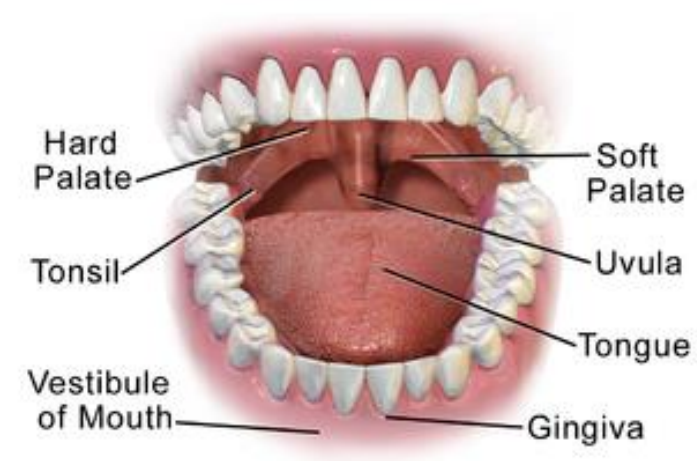

\section{Mouth}

Anatomy of the mouth.

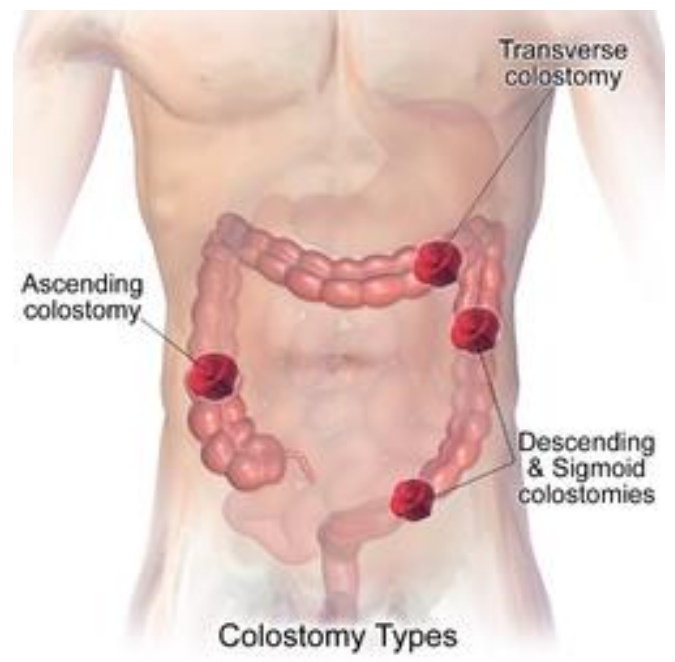

Types of colostomy. 


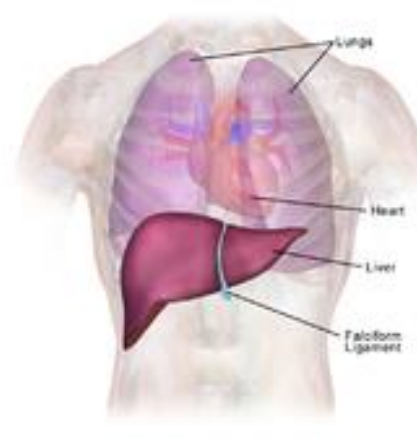

Placenent of the Lher

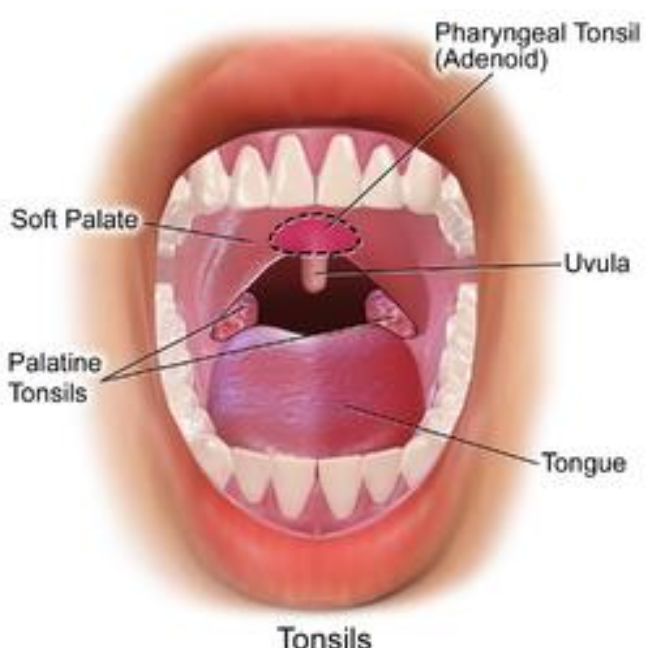

Tonsils and adenoid.

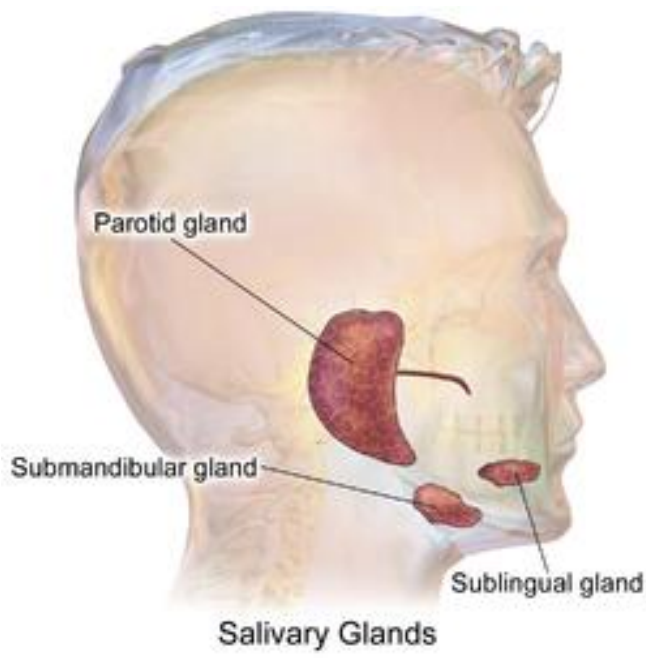

Salivary glands.

\section{Salivary Glands}

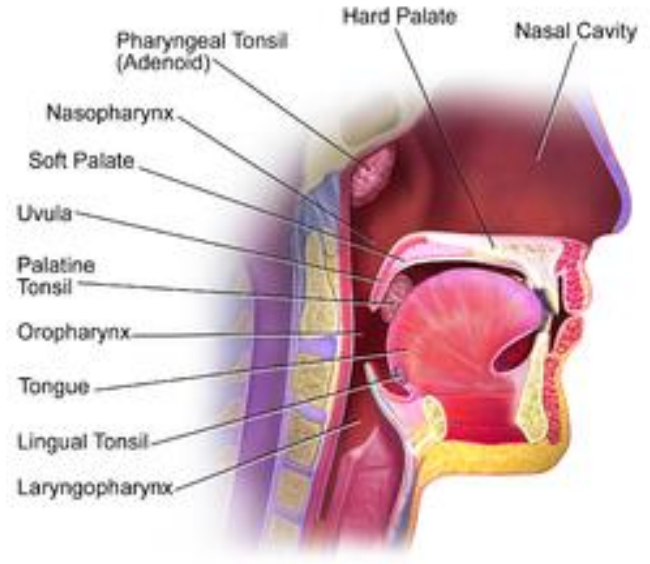

Tonsils and Throat

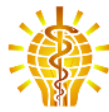

Throat.

Location of the liver. 
๖

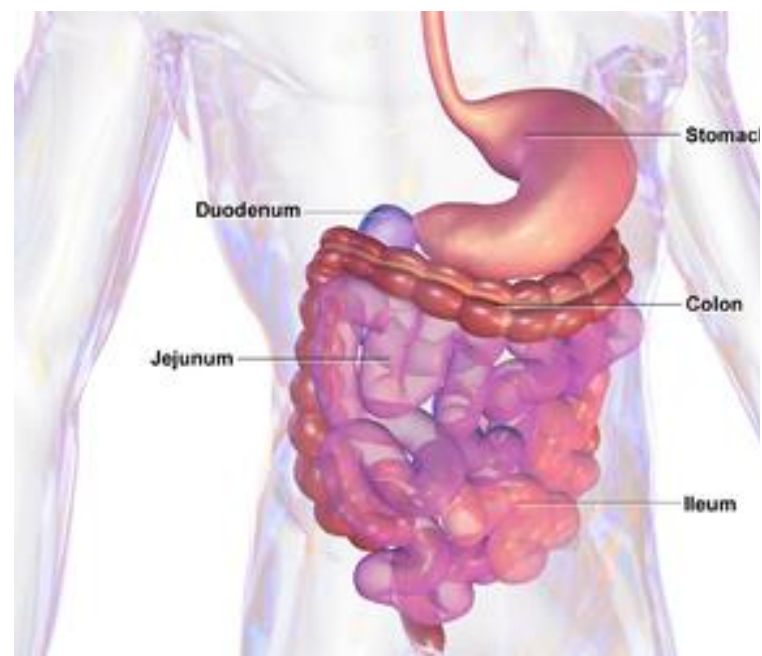

Gastrointestinal system.

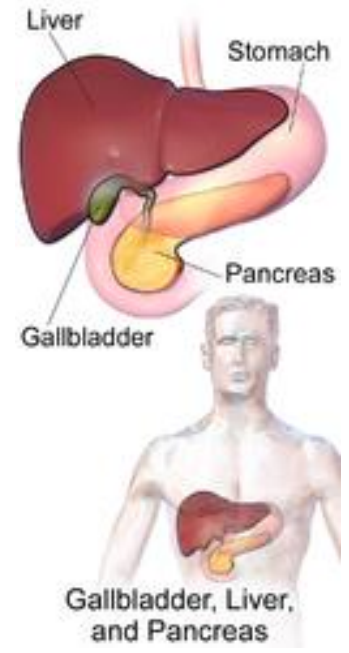

Gallbladder, liver and pancreas
WikiJournal of Medicine, 2014, 1(2) doi: $10.15347 / \mathrm{wjm} / 2014.010$

Figure Article
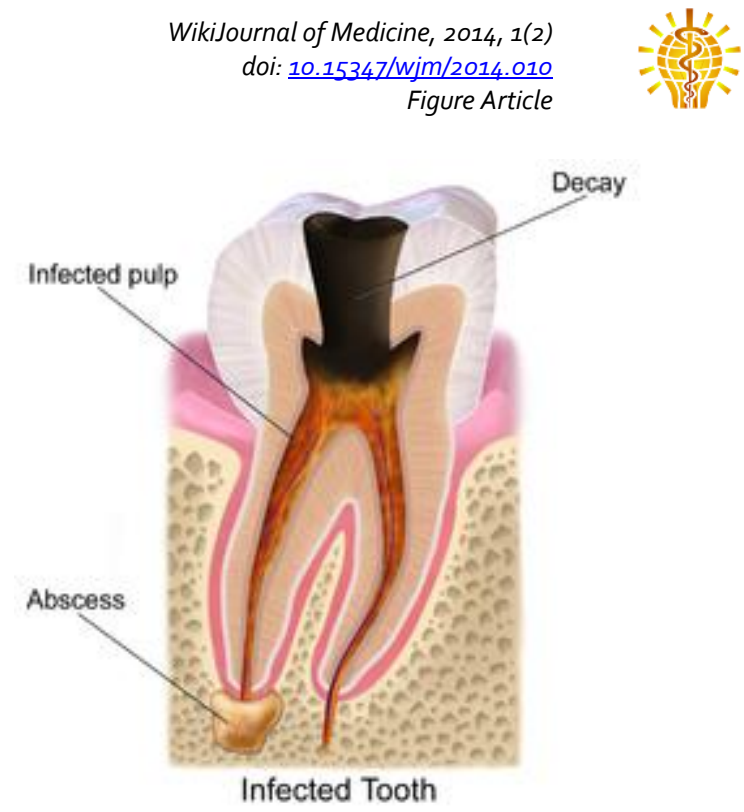

Tooth decay.

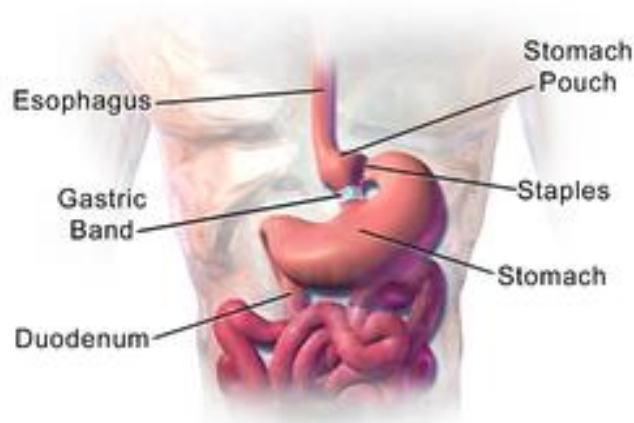

Vertical Banded Gastroplasty

Vertical banded gastroplasty surgery. 


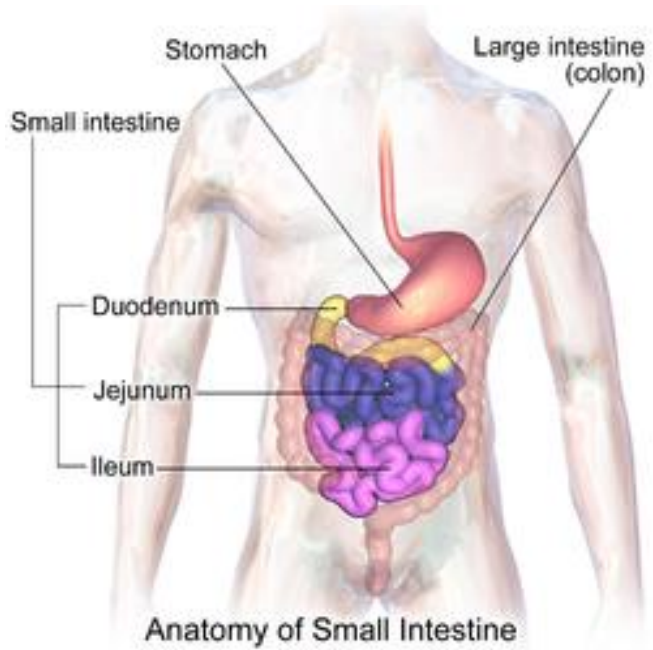

Small intestine anatomy

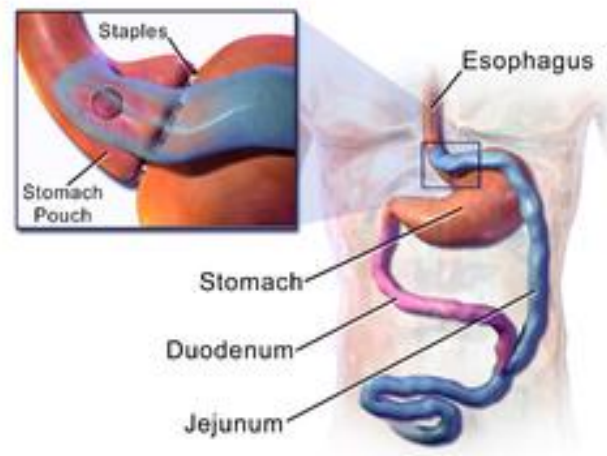

Roux-En-Y

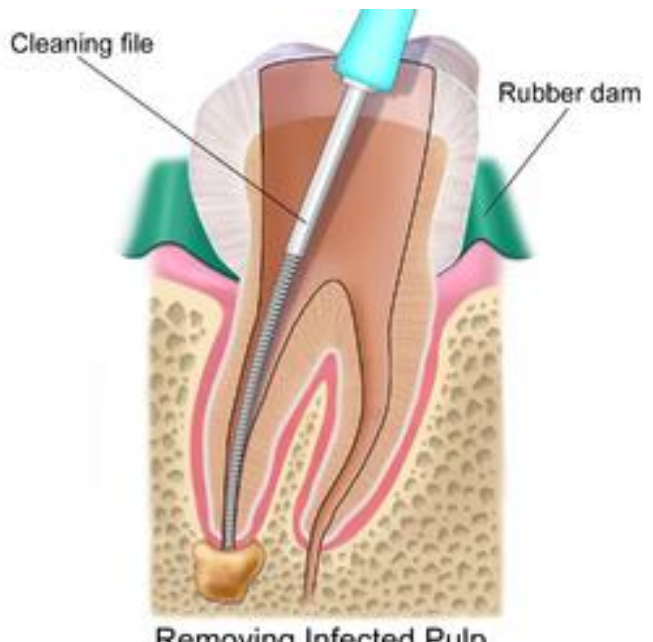

Removing Infected Pulp

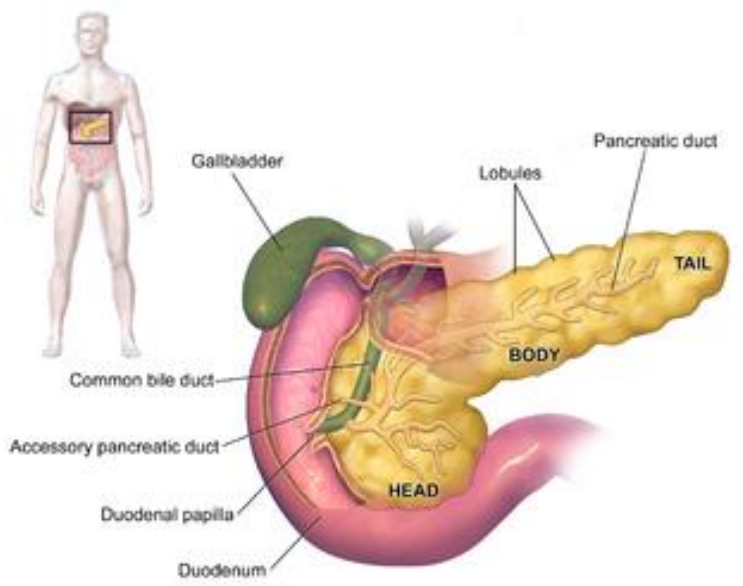

Pancreas anatomy

Roux-en-Y anastomosis. 


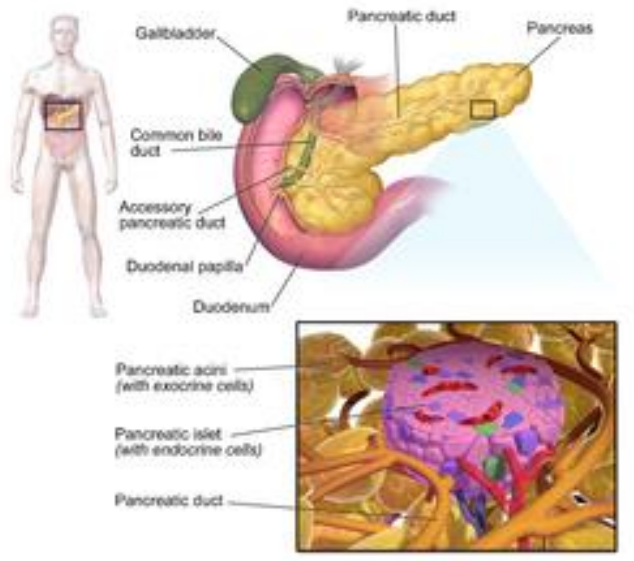

Pancreatic Tissue

Pancreatic tissue
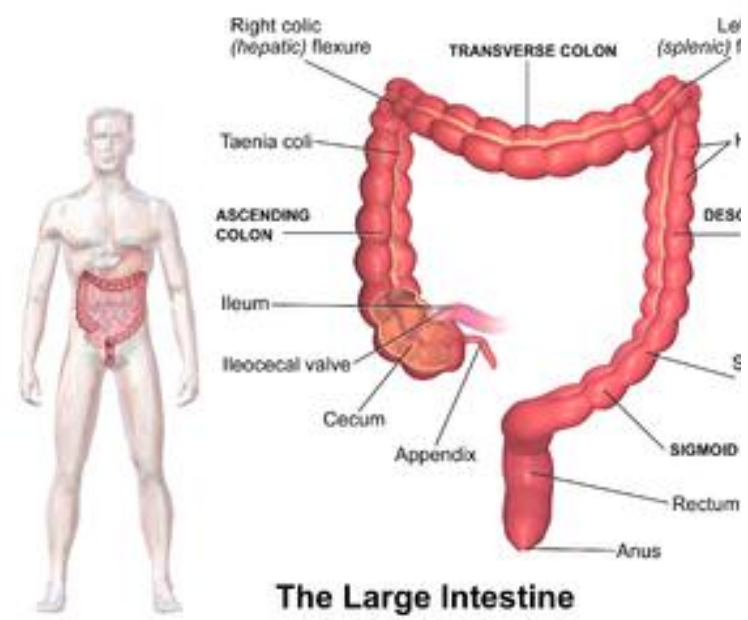

The Large Intestine

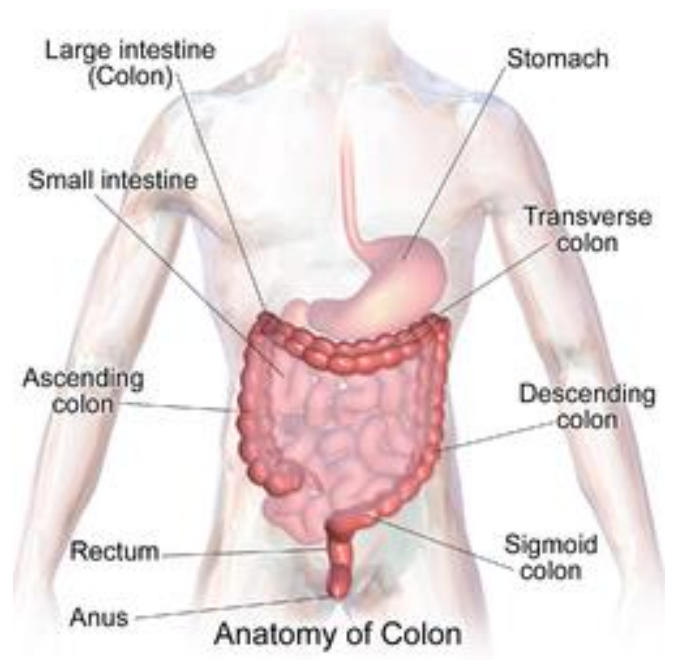

Large intestine.

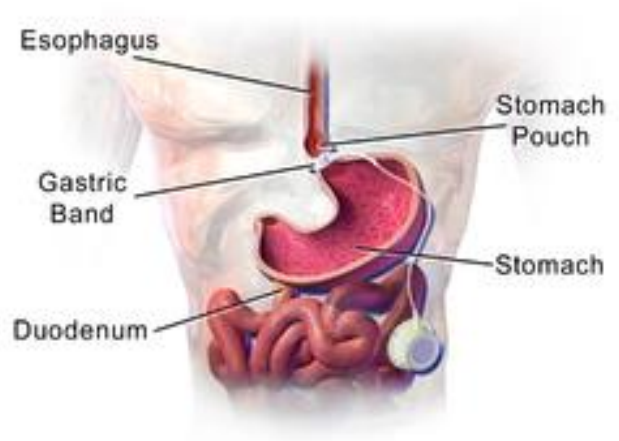

Adjustable Gastric Banding

Large intestine.

Adjustable gastric banding 


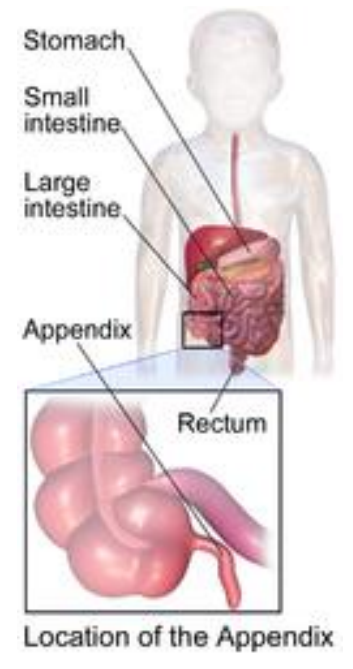

Appendix in a child.

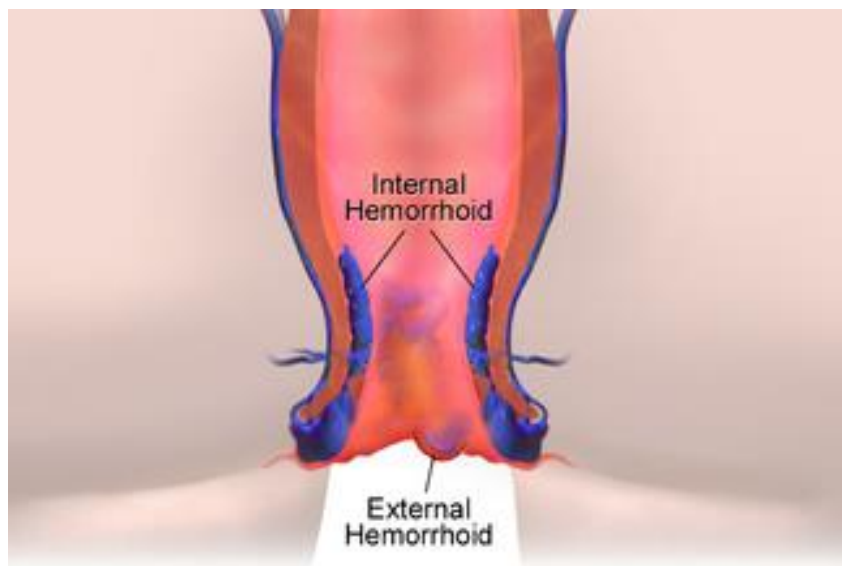

\section{Anus With Hemorrhoids}




\section{Neurology}

An Introduction to Brain Structures

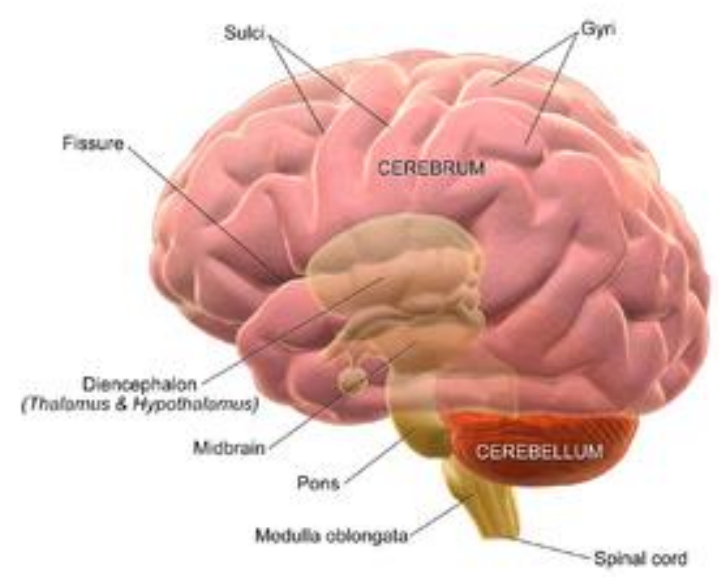

Brain structures.

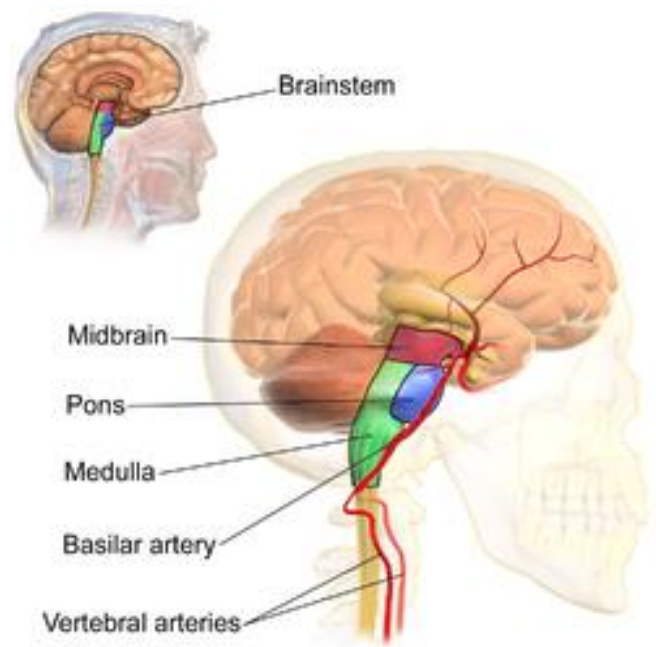

Brain stem anatomy.

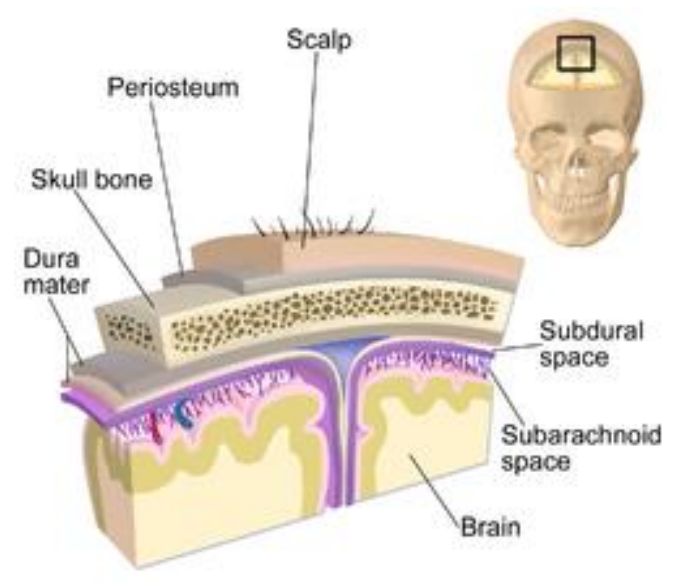

Layers covering the Brain

Layers of the scalp. 


\section{Motor and Sensory Regions of the Cerebral Cortex}

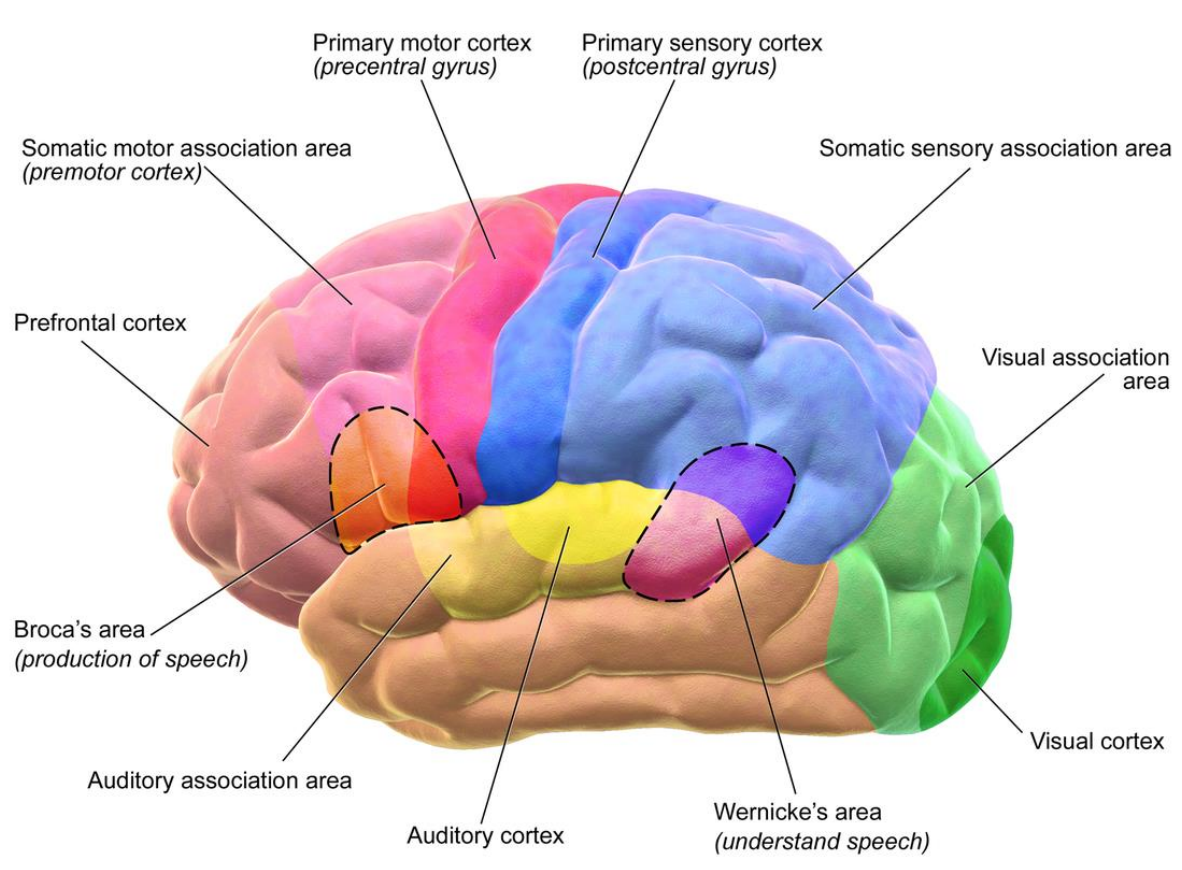

Regions of the cerebral cortex.

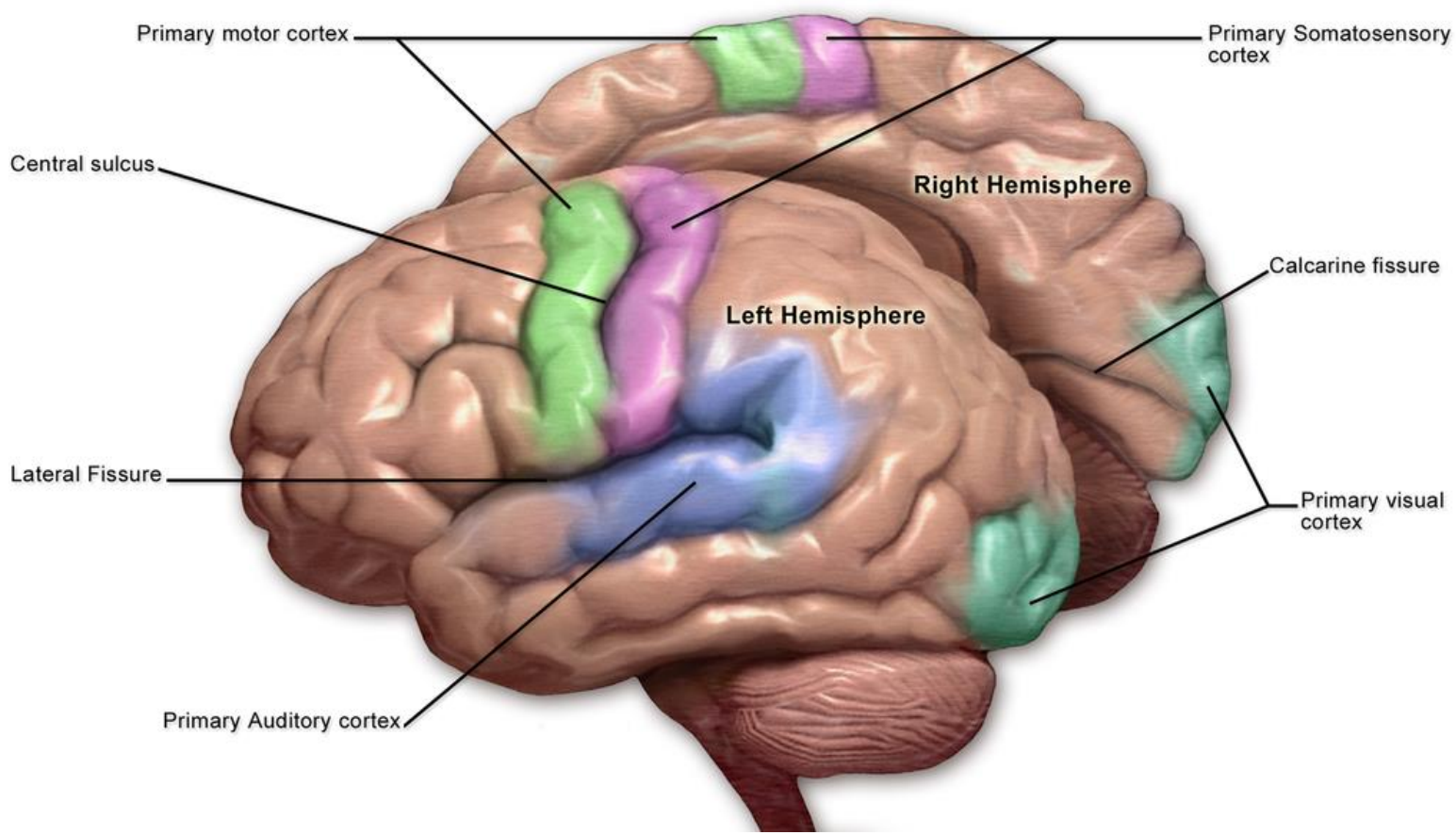

Primary motor, somatosensory, auditory and visual cortex. 


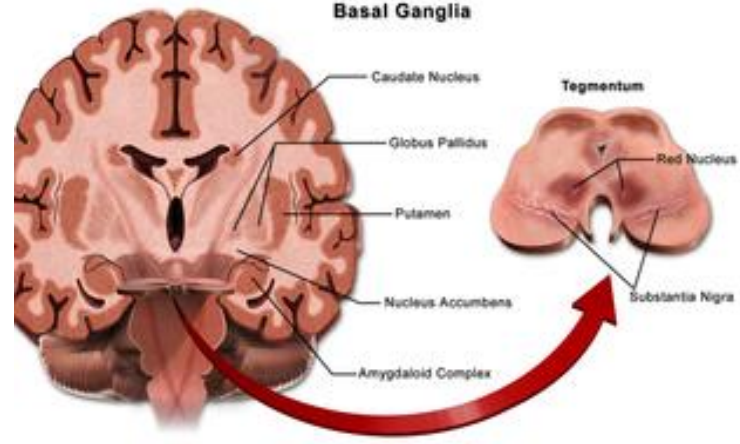

Basal ganglia.

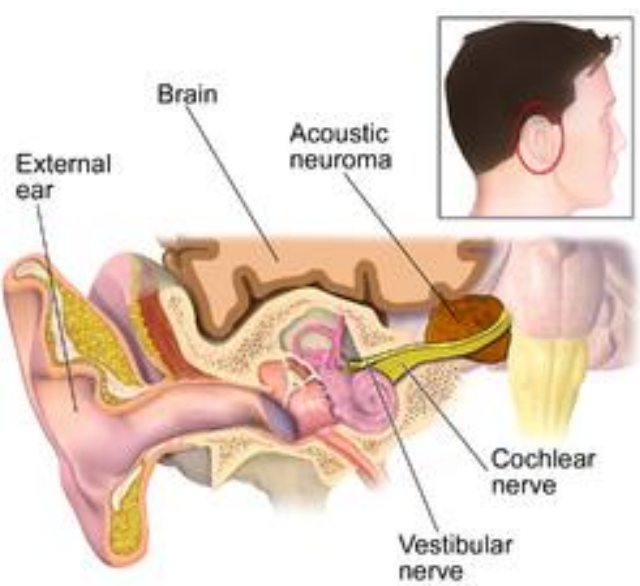

Acoustic Neuroma

Acoustic neuroma.
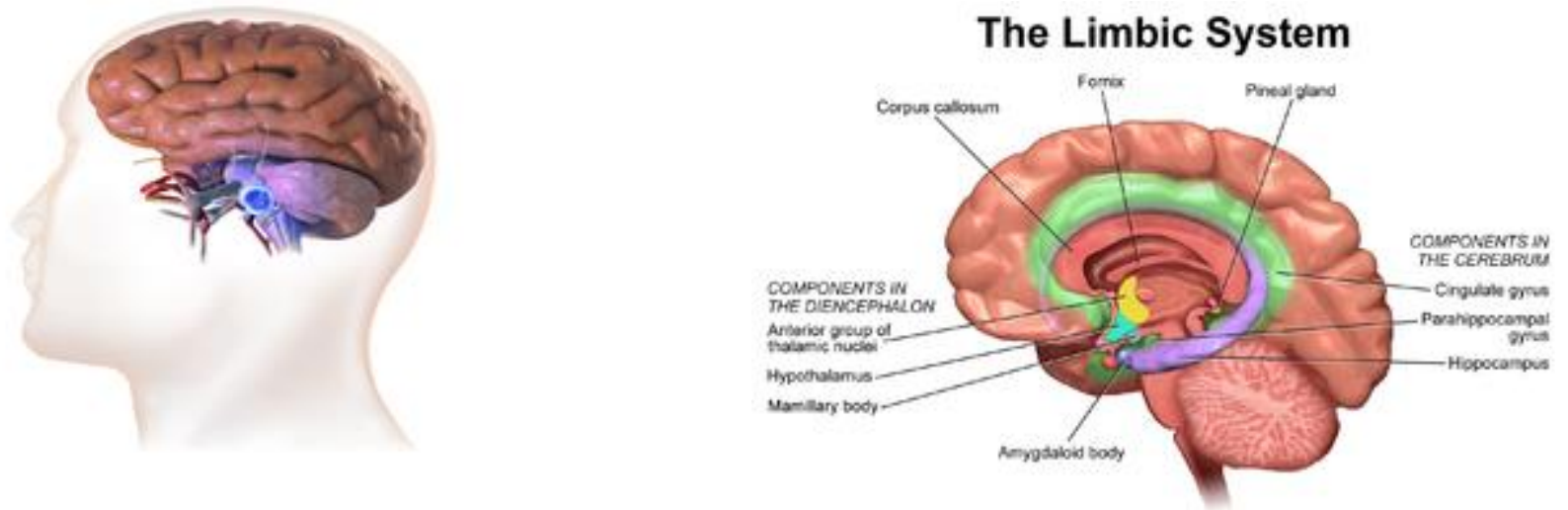

Acoustic neuroma.

Limbic system. 
웅

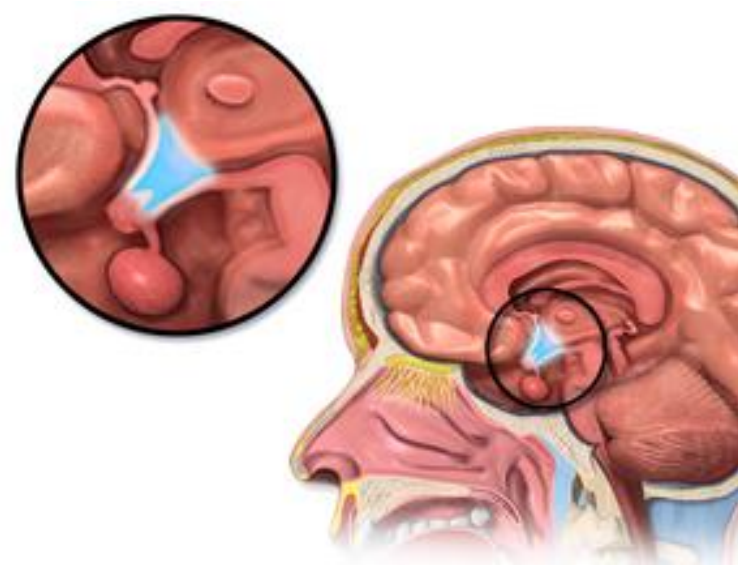

Hypothalamus location.

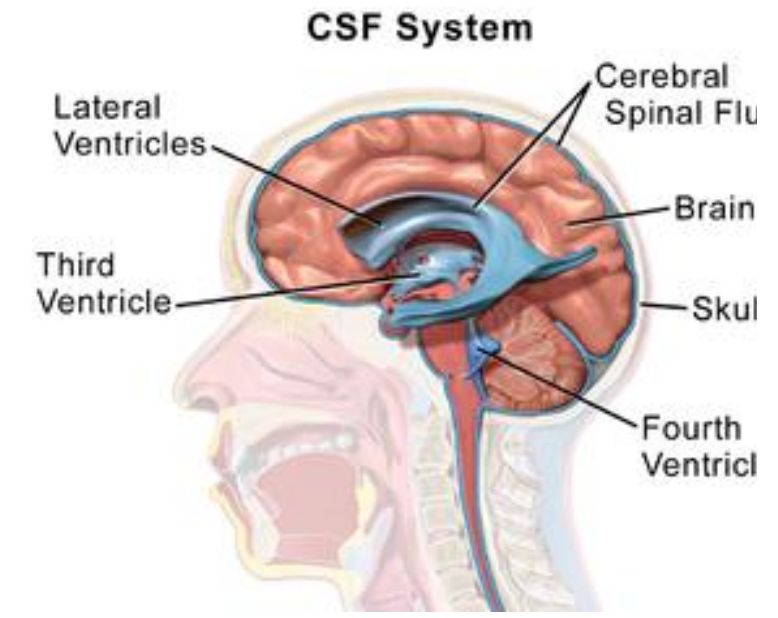

Ventricular system.
WikiJournal of Medicine, 2014, 1(2) doi: $10.15347 / \mathrm{wjm} / 2014.010$

Figure Article

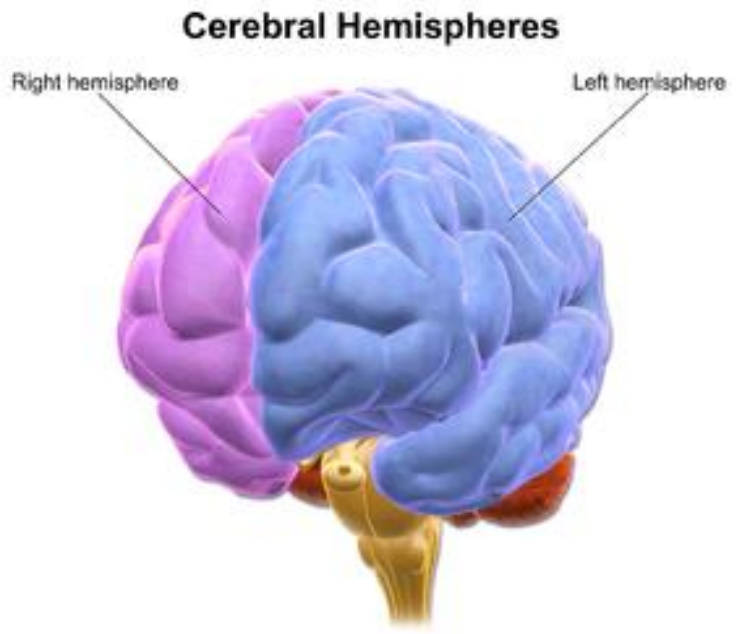

Cerebral hemispheres

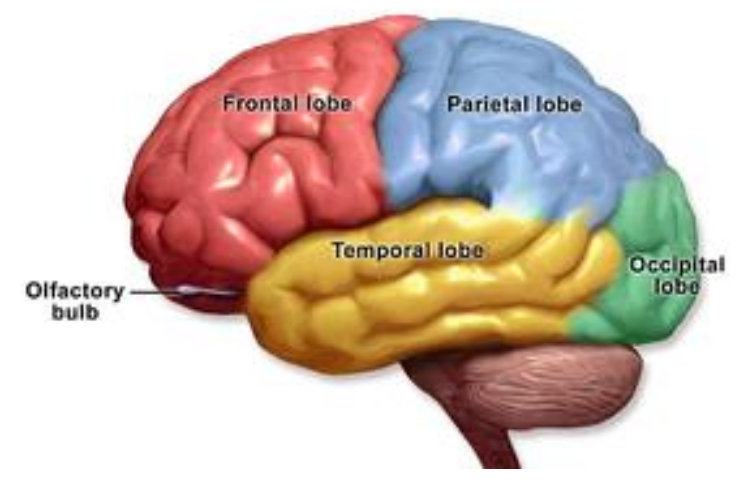

Brain lobes.

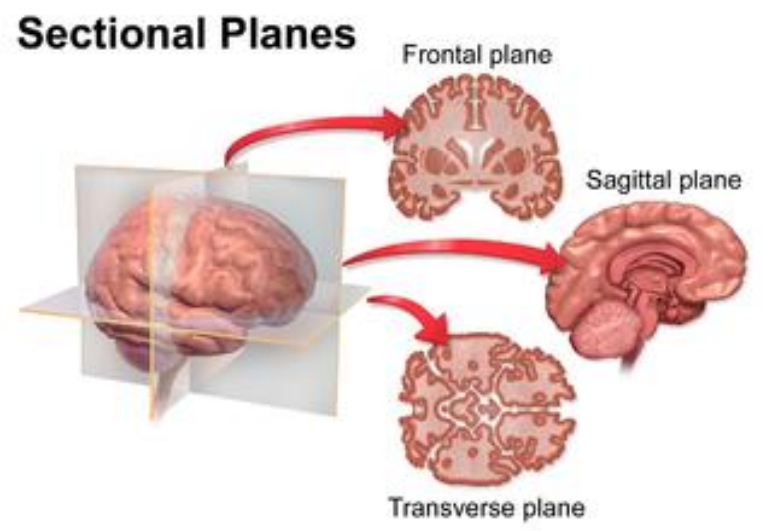

Anatomical planes of the brain. 


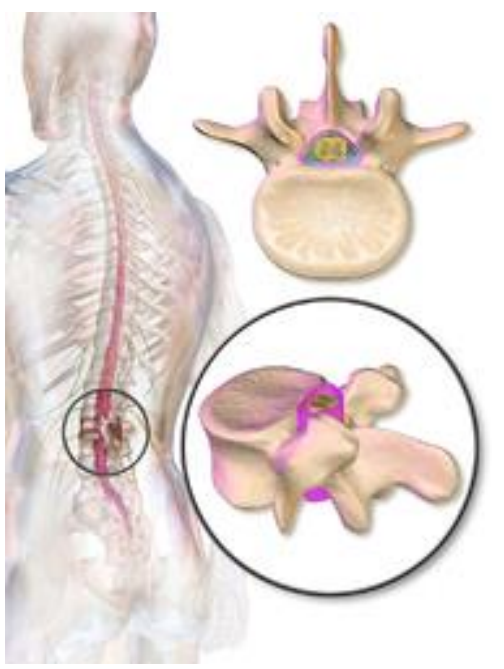

Dura mater of the spine.

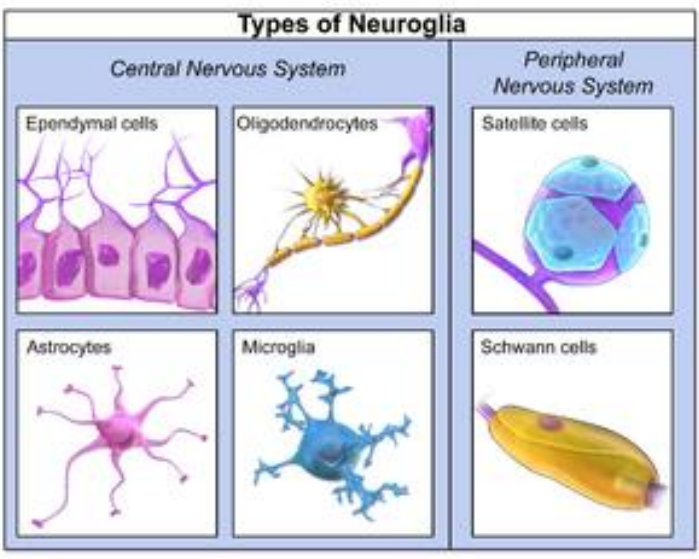

Types of neuroglia cells.

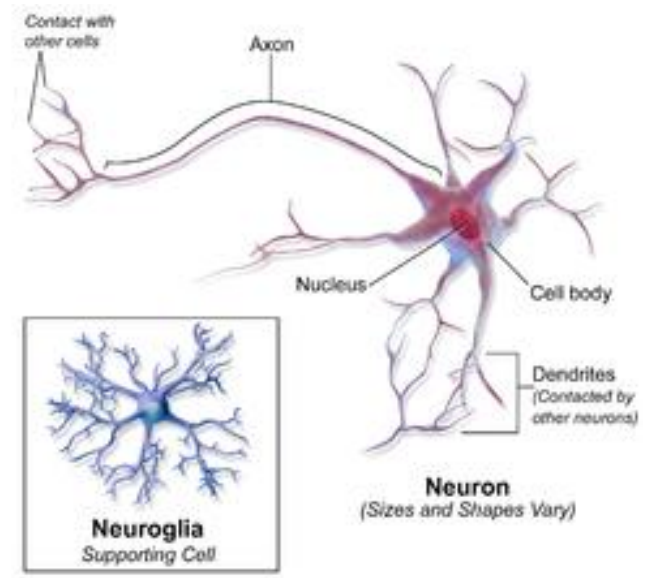

Neural Tissue

Nervous tissue.

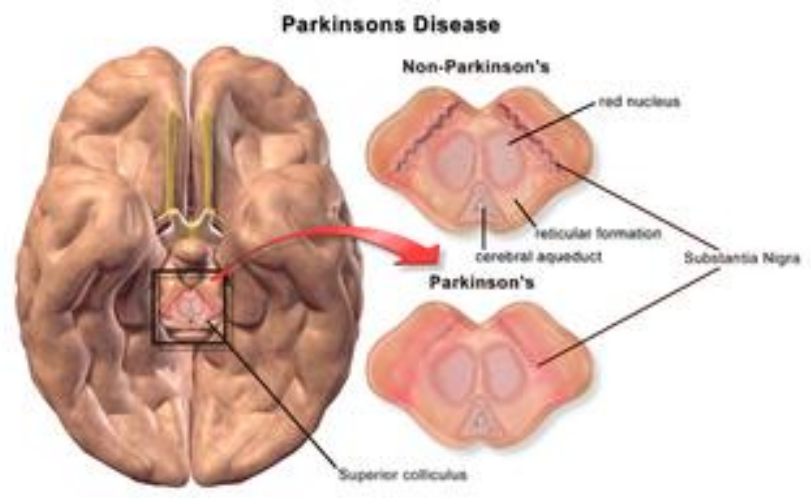

Parkinson's disease. 


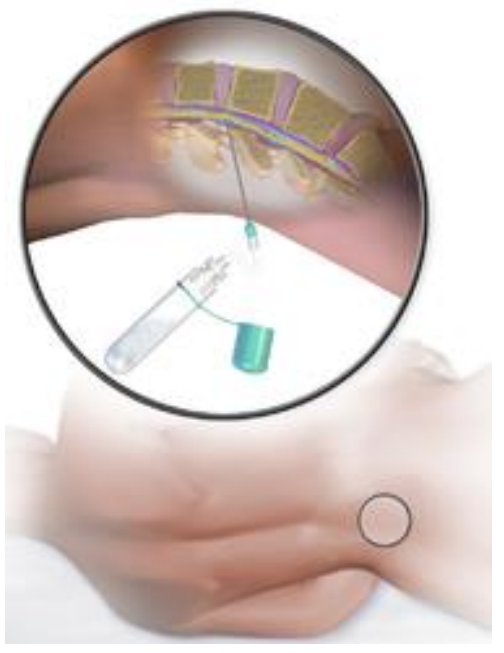

Lumbar puncture.

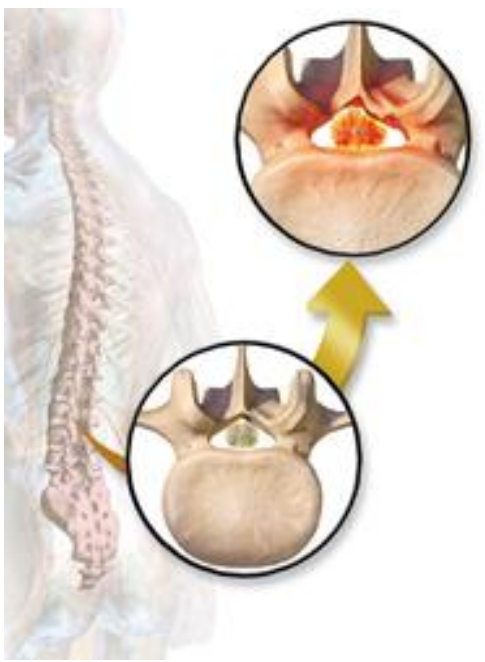

Spinal stenosis.

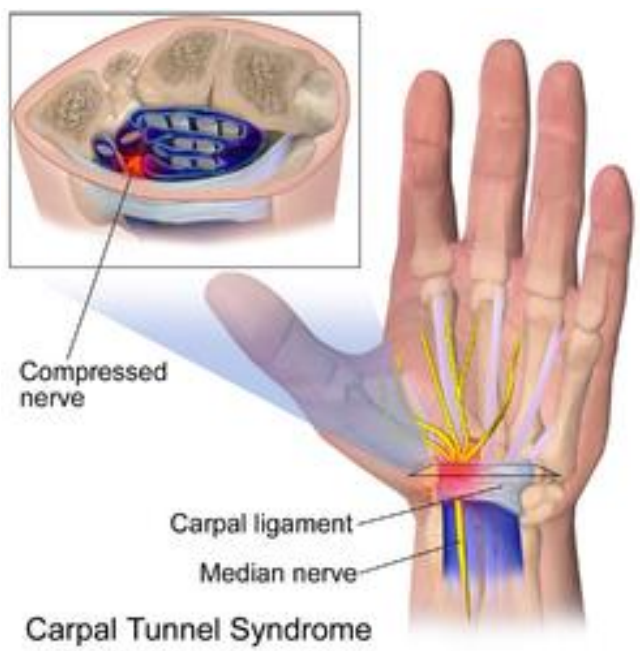

Carpal tunnel syndrome.

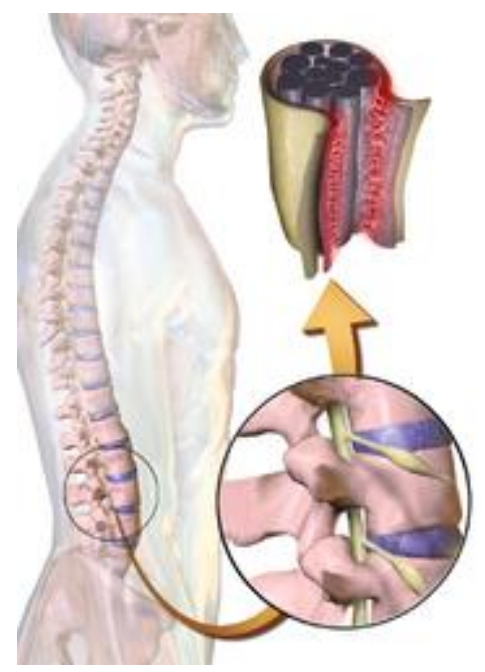

Arachnoiditis. 


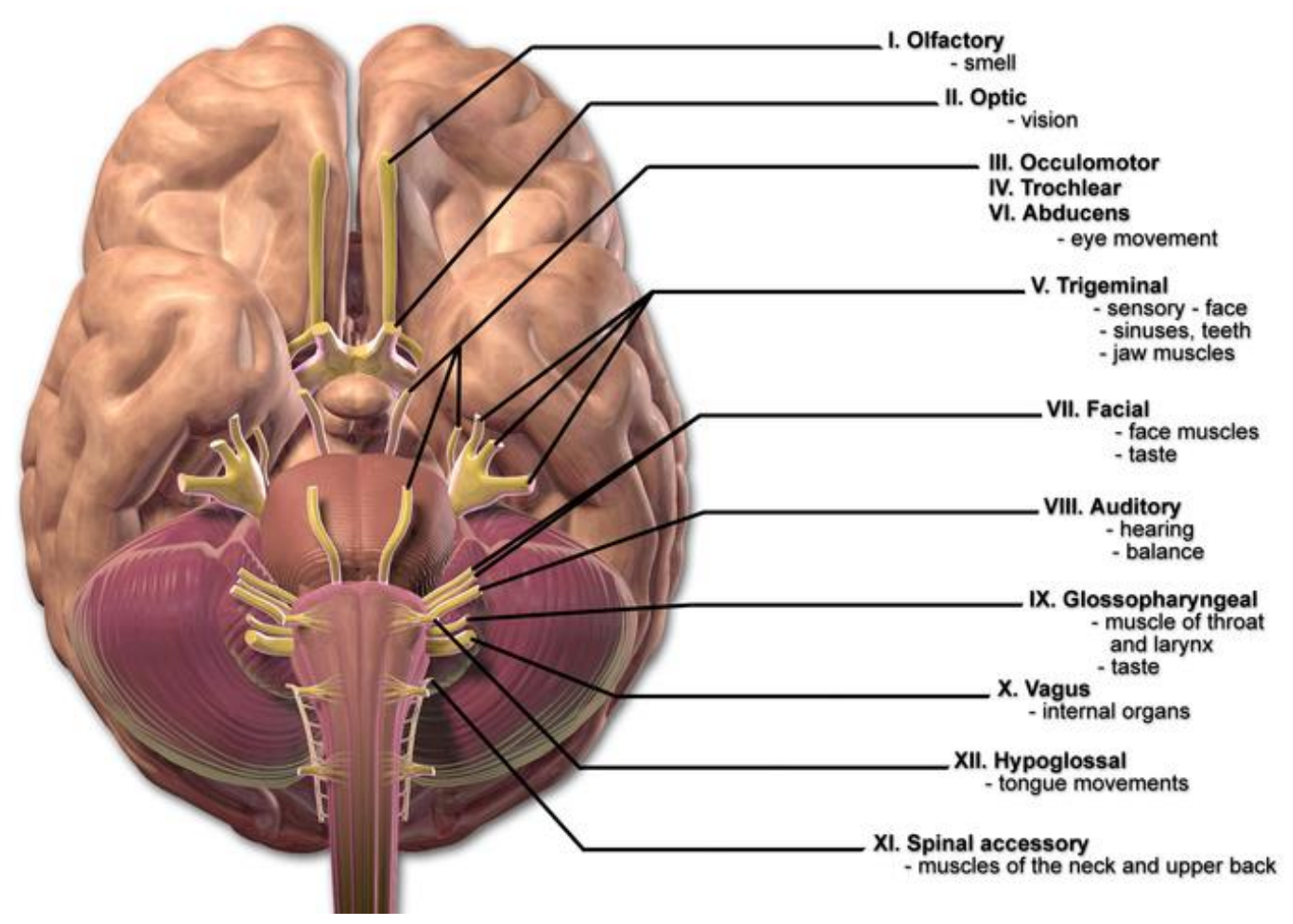

Cranial nerves.

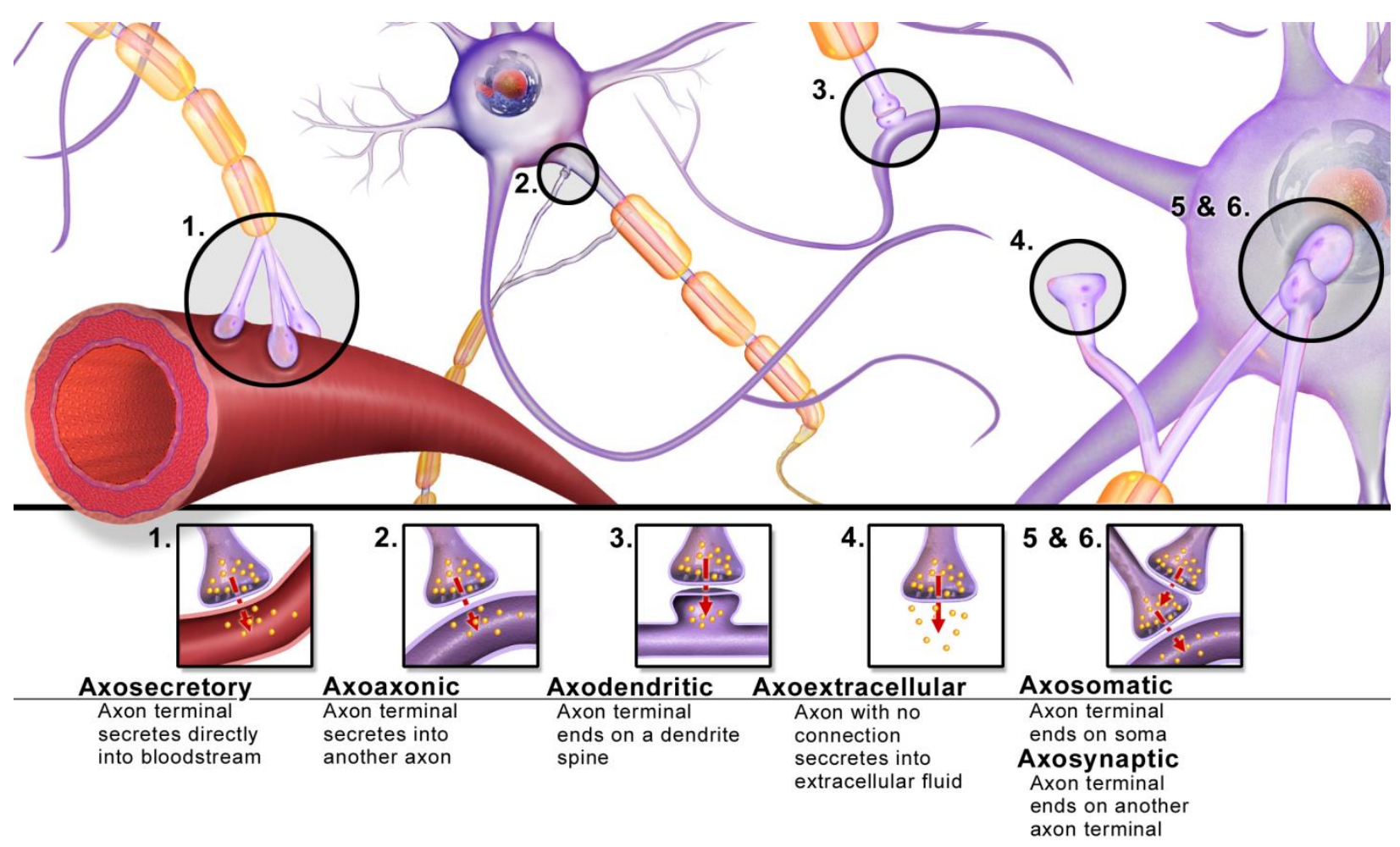

Synapse types. 


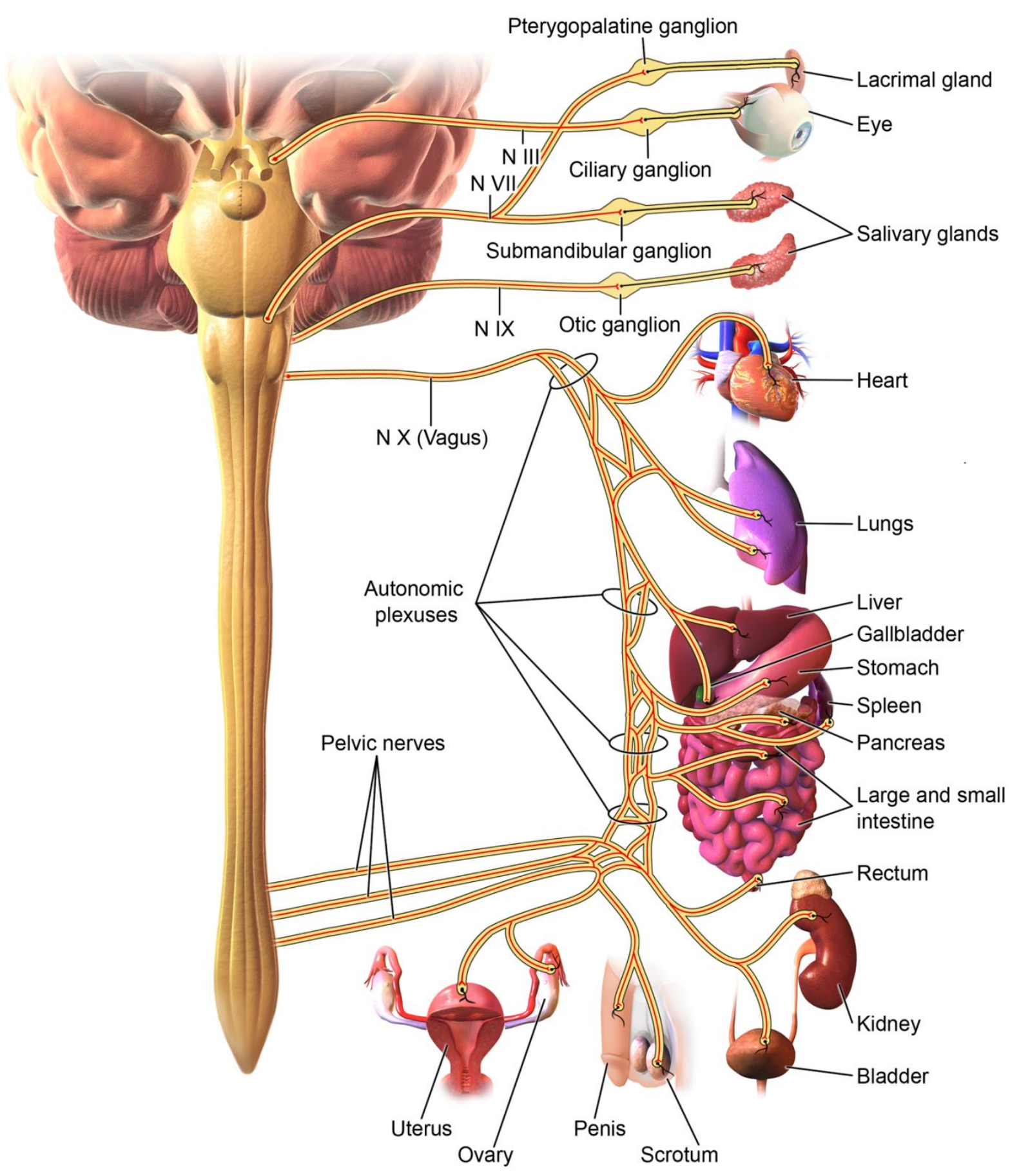

\section{Parasympathetic Innervation}

Innervation of the parasympathetic system. 


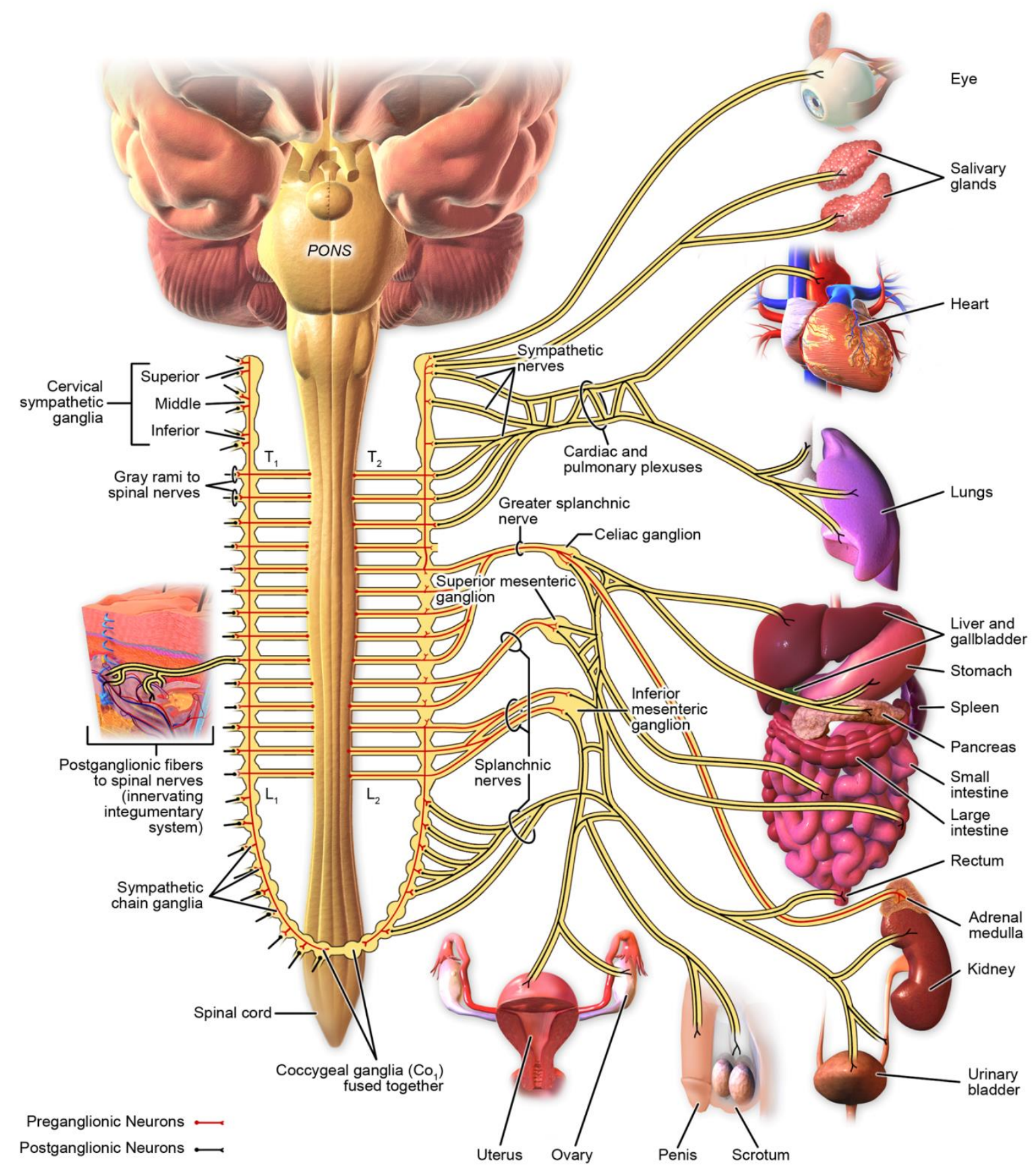

\section{Sympathetic Innervation}

Innervation of the sympathetic system. 


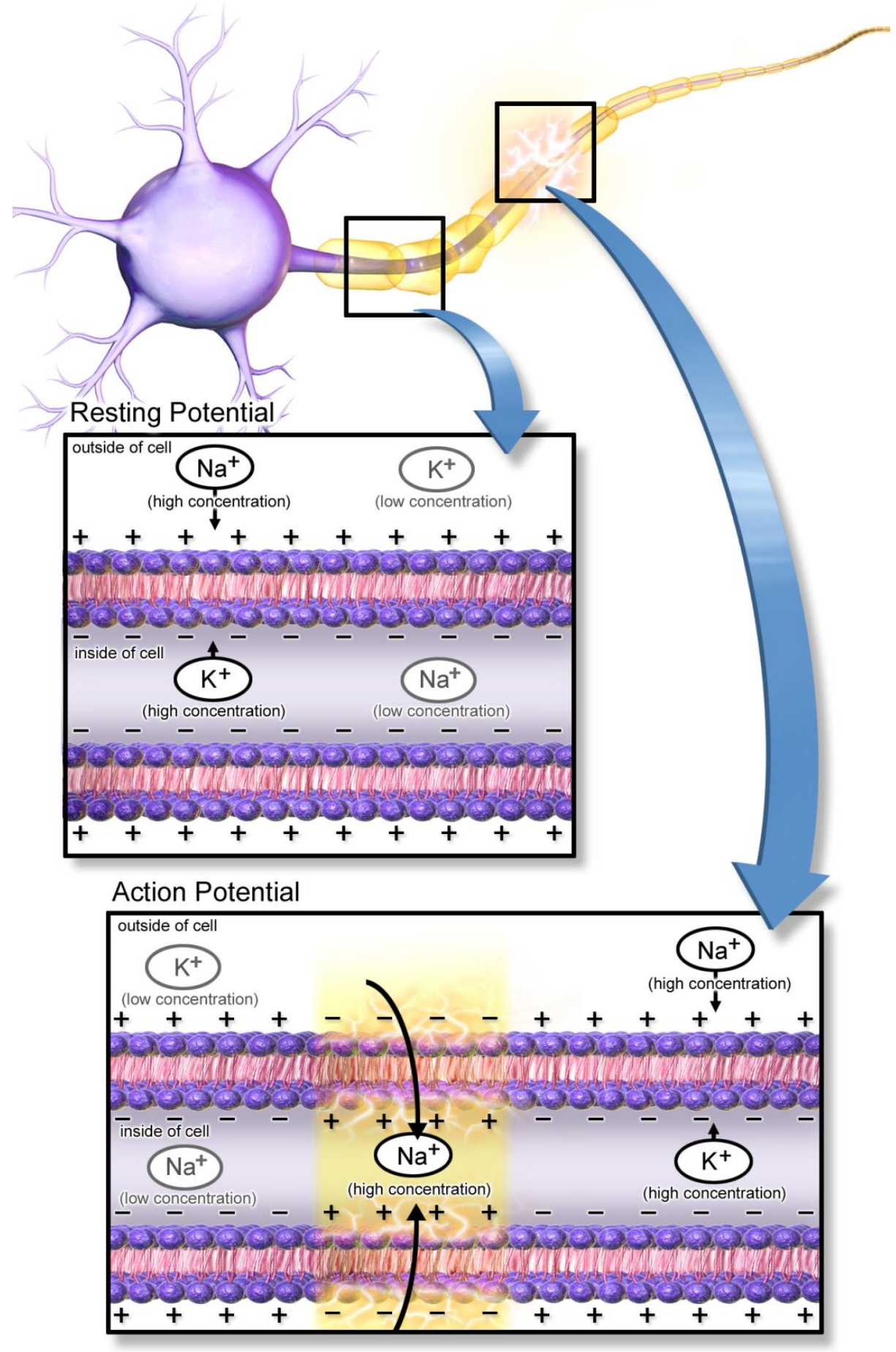

Action potential. 


\section{Vertebral}

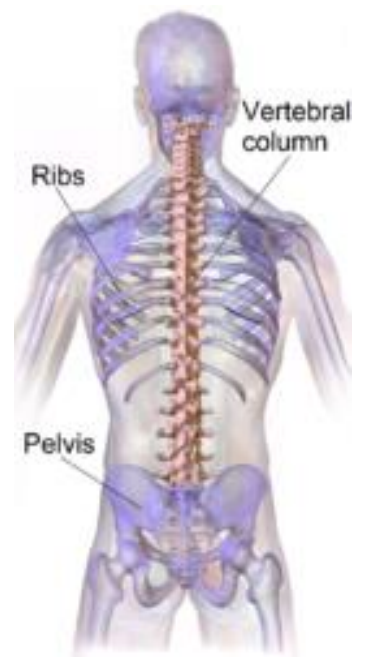

Normal vertebral column.

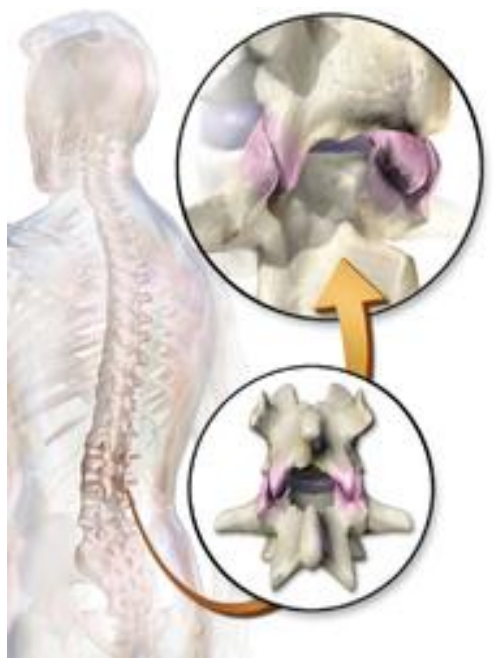

Facet joints.

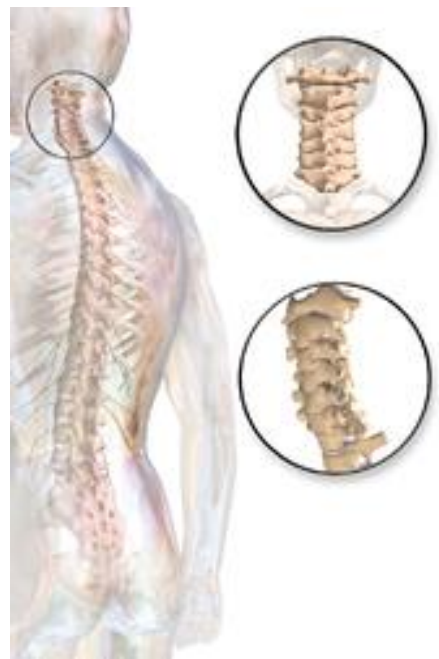

Cervical spine.

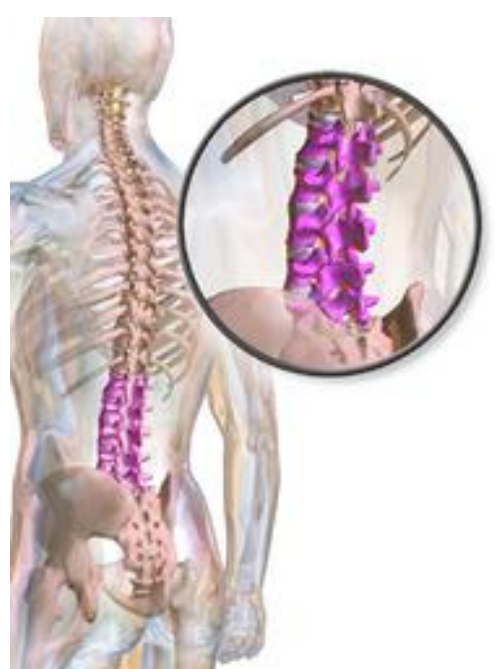

Lumbar spine. 


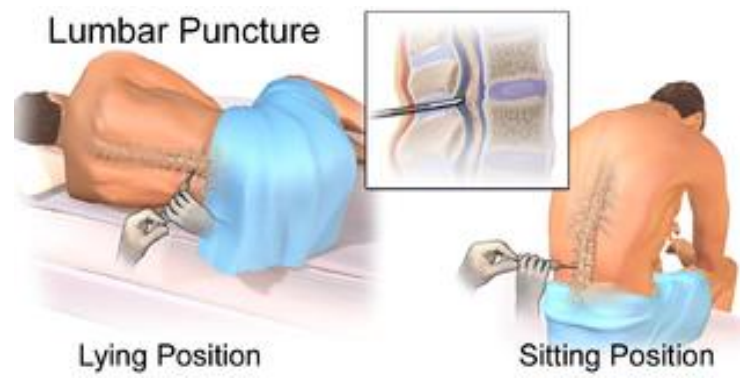

Lumbar puncture.

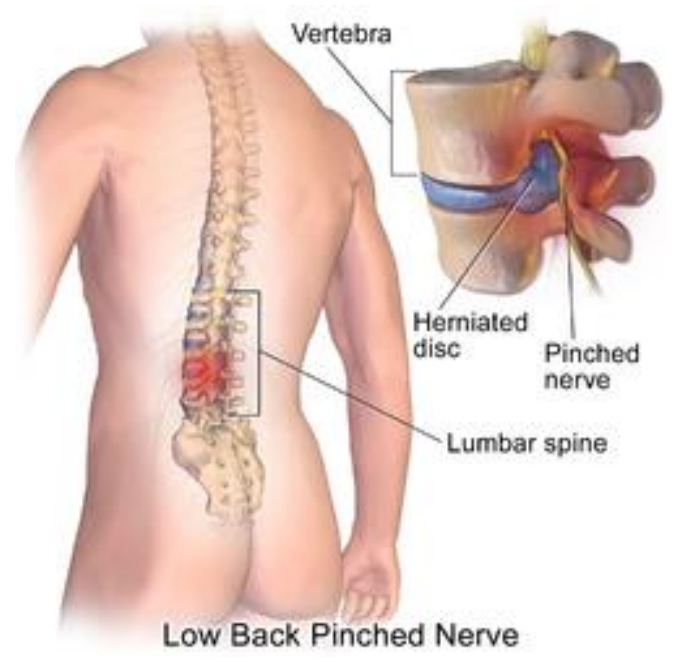

Lumbar disc herniation

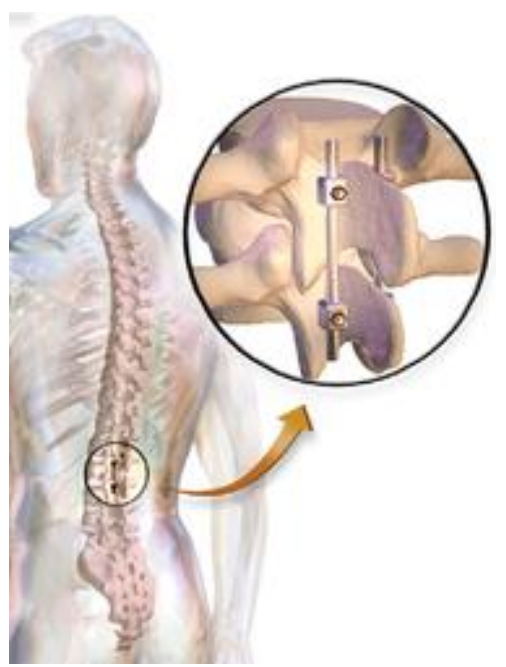

Harrington rods

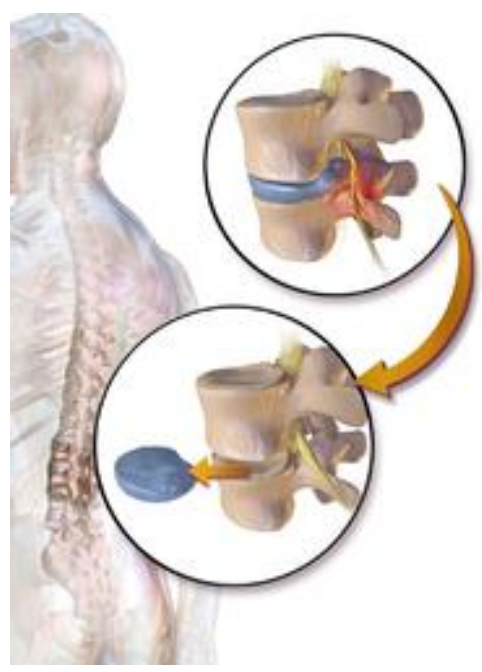

Discectomy.

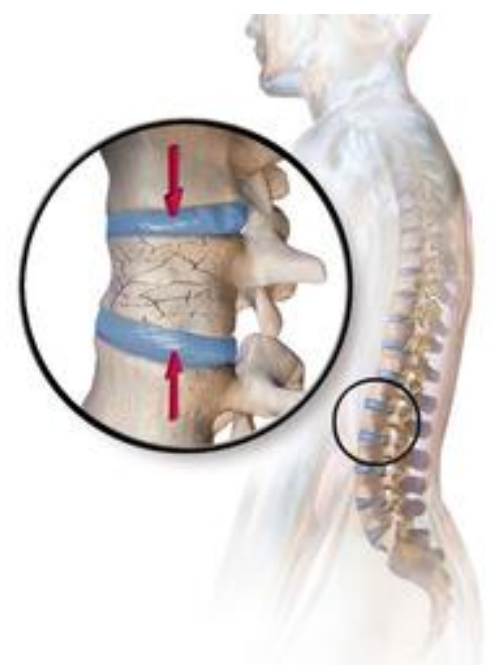

Compression fracture. 


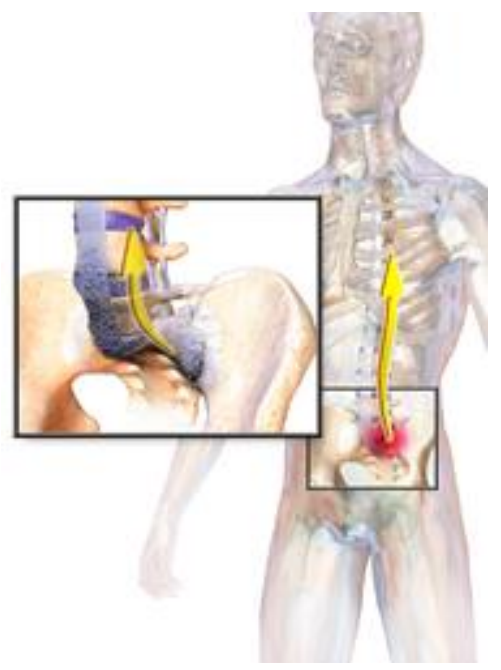

Ankylosing spondylitis.

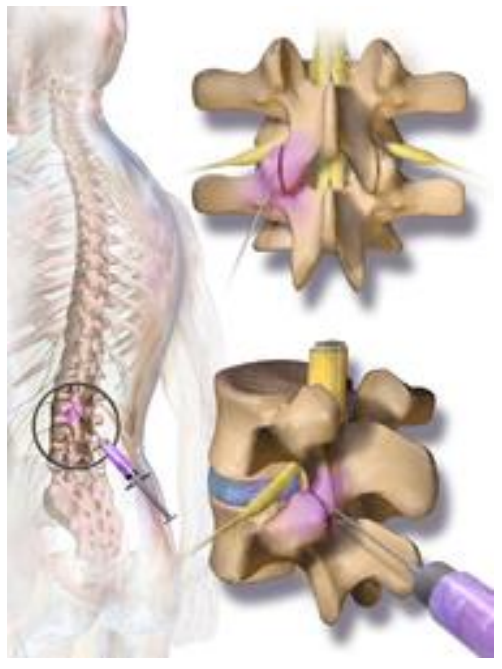

Facet joint injection.

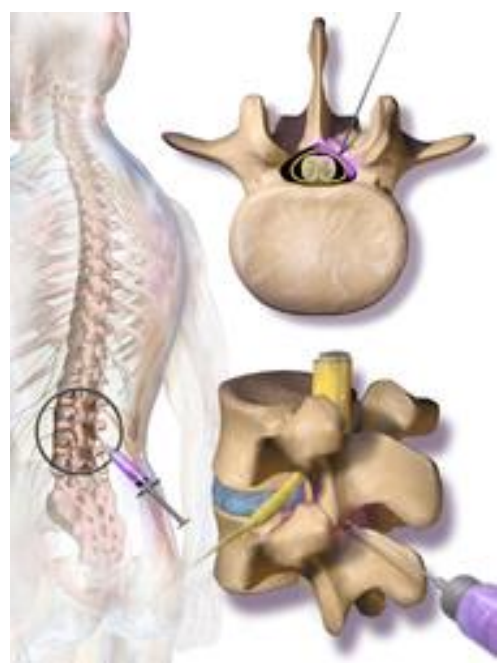

Epidural steroid injection.

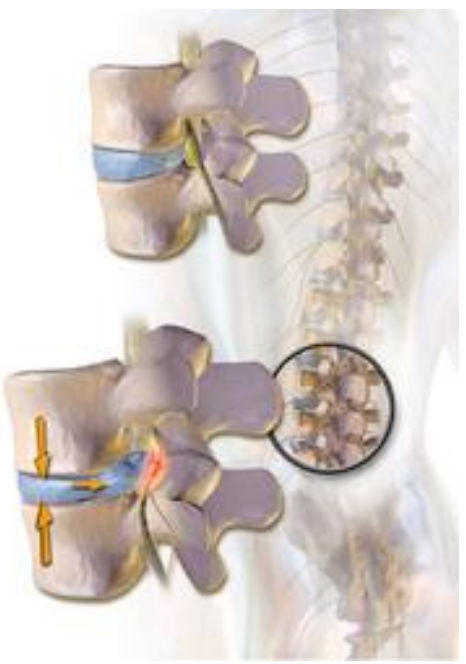

Spinal disc herniation. 


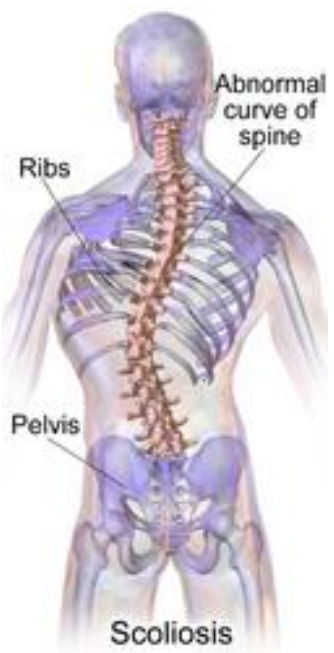

Scoliosis. 


\section{Obstetrics and gynecology}

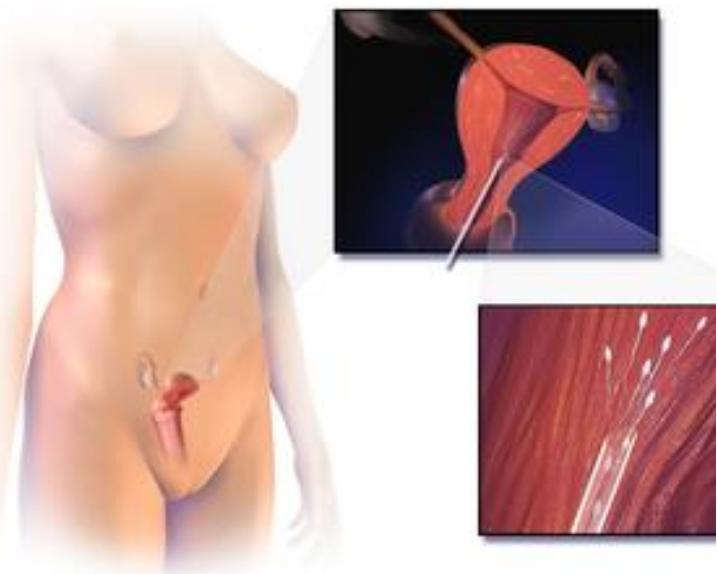

Artificial insemination.

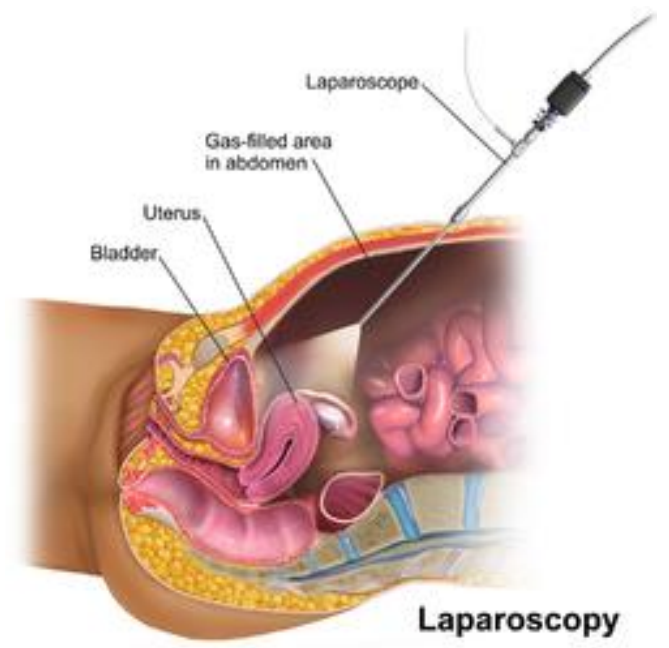

Laparoscopy.

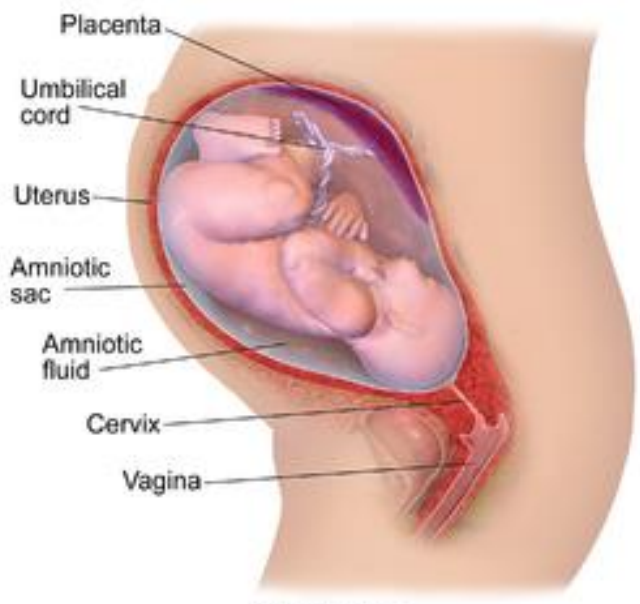

Pregnancy

Pregnancy.

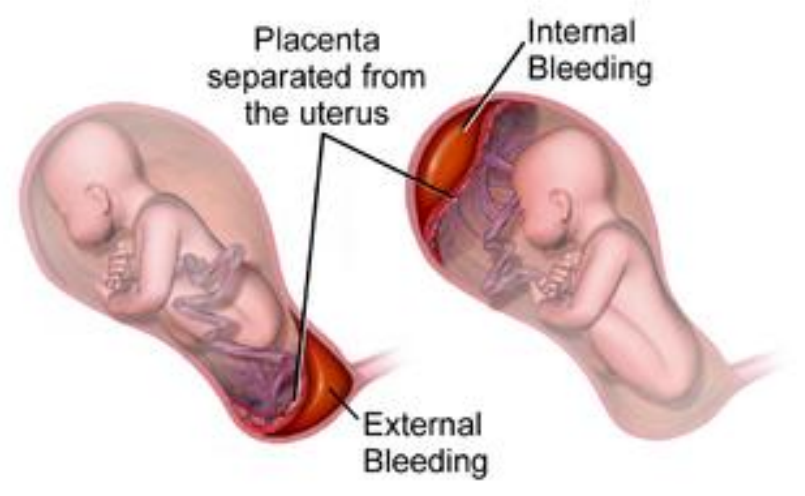

Abruptio Placenta (Placental Abruption)

Placental abruption. 
우
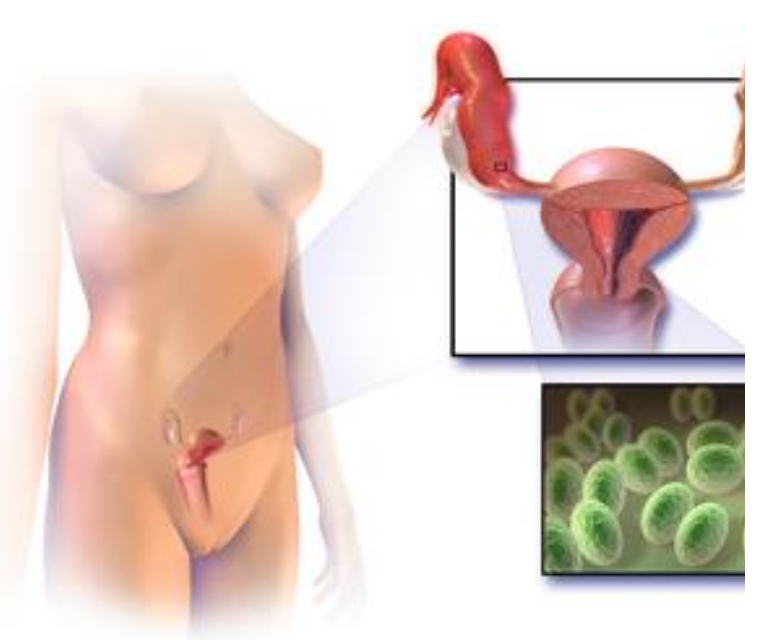

Pelvic inflammatory disease.

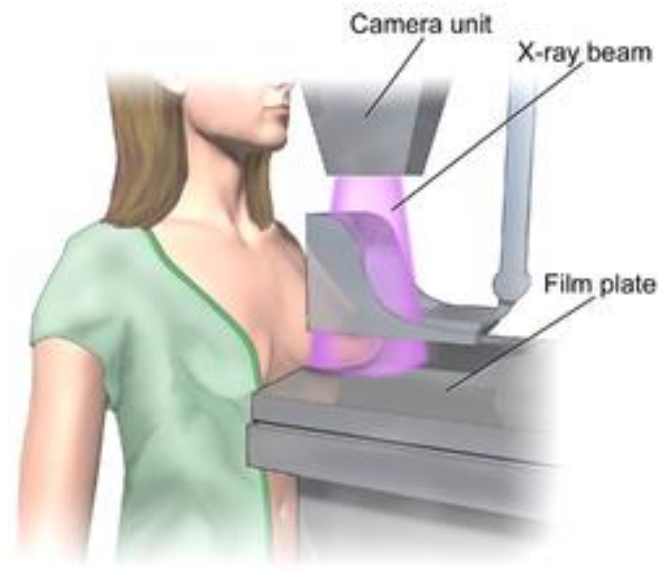

Mammogram
WikiJournal of Medicine, 2014, 1(2) doi: $10.15347 / \mathrm{wjm} / 2014.010$

Figure Article

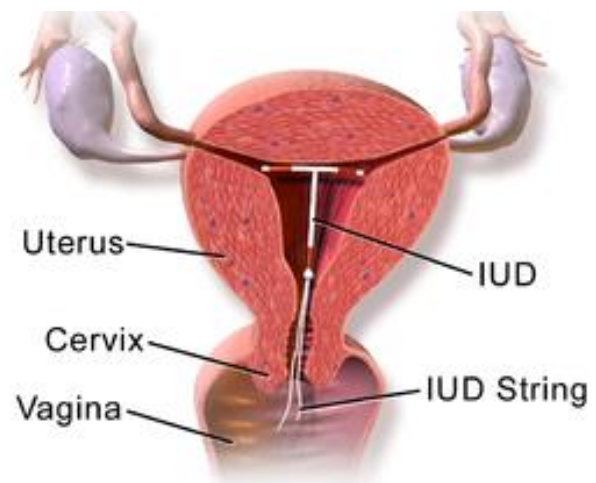

Intraunterine Device (IUD)

Intrauterine device.

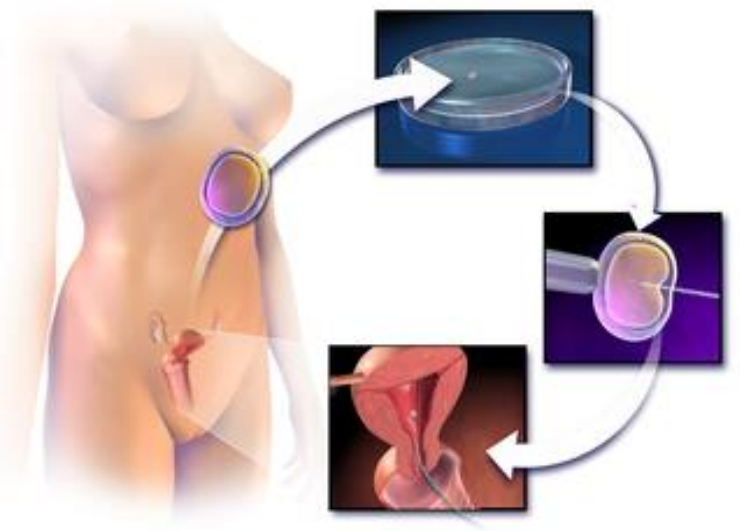

In vitro fertilization (IVF) with intracytoplasmic sperm injection (ICSI).

Mammography. 
우

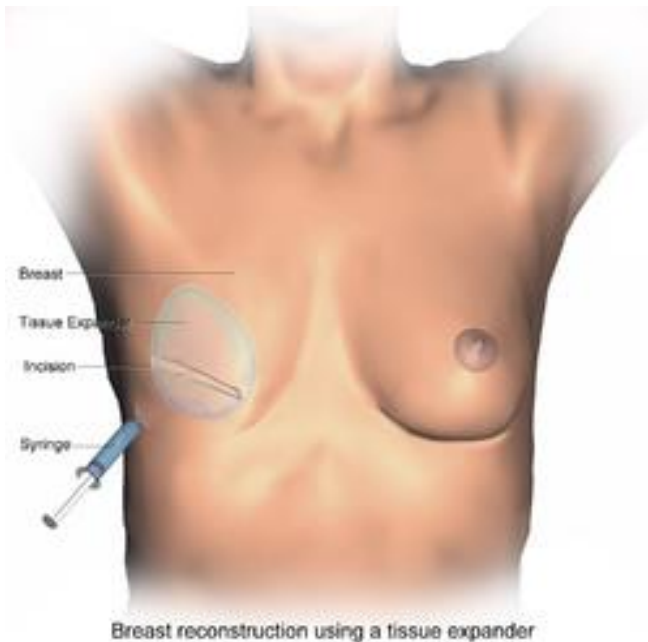

Breast reconstruction using a tissue expander.

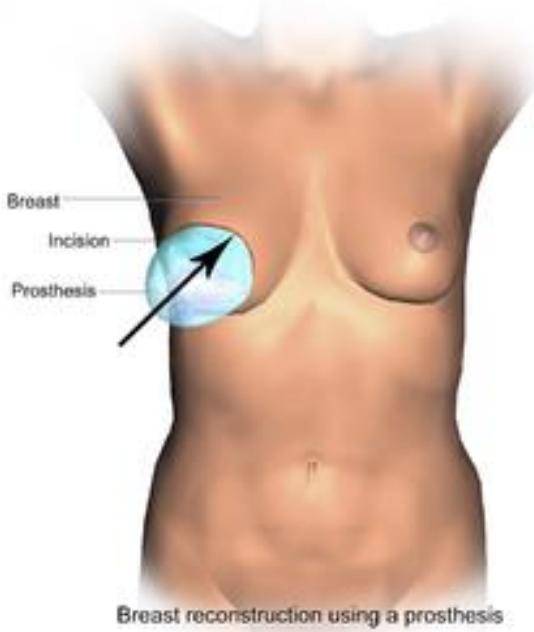

Breast reconstruction using a prosthesis.
WikiJournal of Medicine, 2014, 1(2) doi: $10.15347 / \mathrm{wjm} / 2014.010$

Figure Article

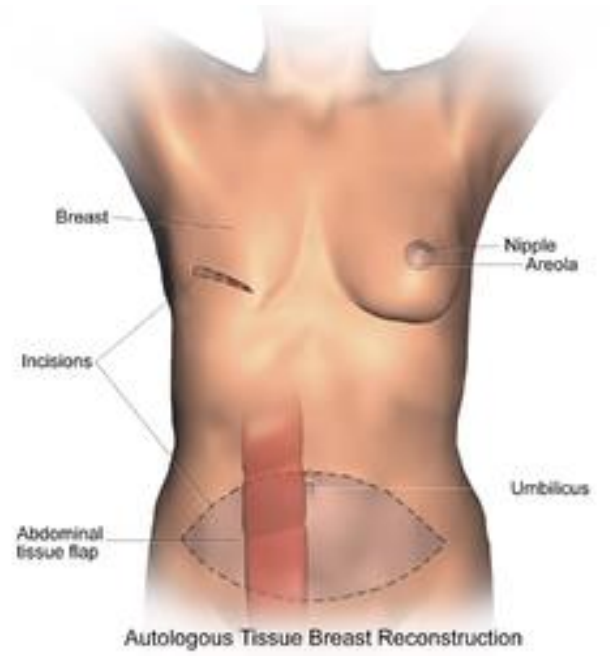

Breast reconstruction using autologous tissue (TRAM).

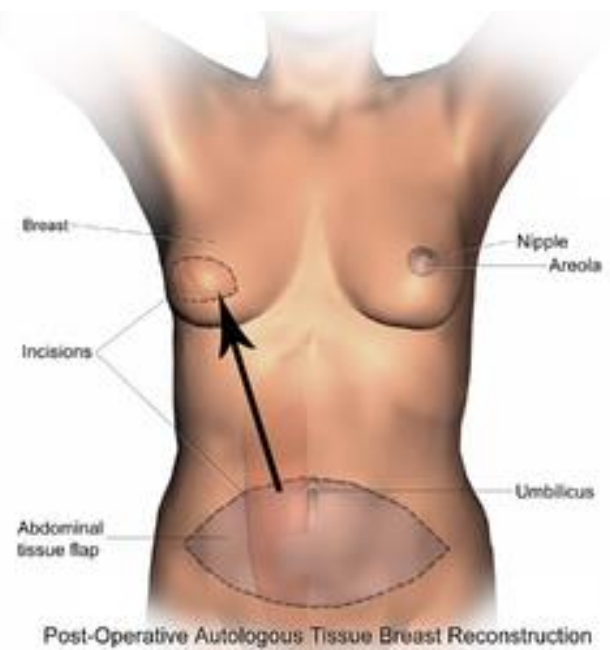

Post-operative state after breast reconstruction usingautologous tissue (TRAM). 
๖ ๔

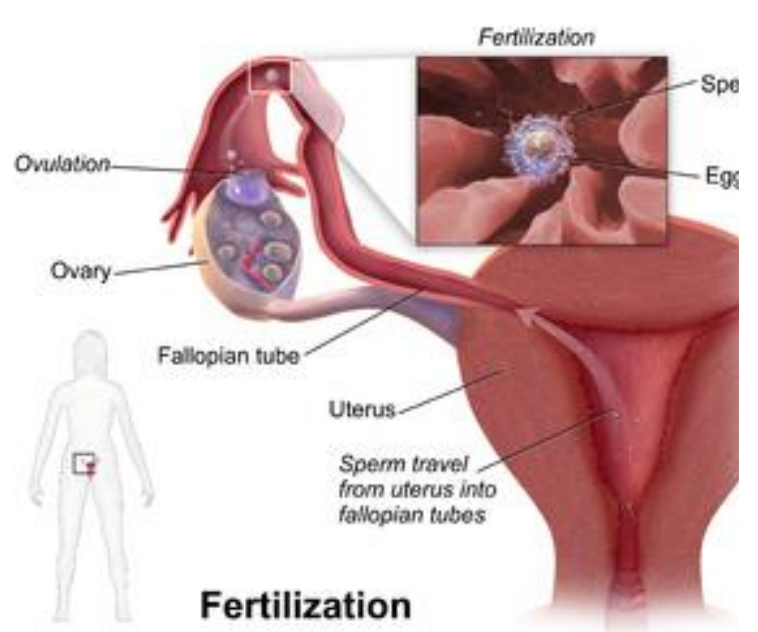

Fertilization.

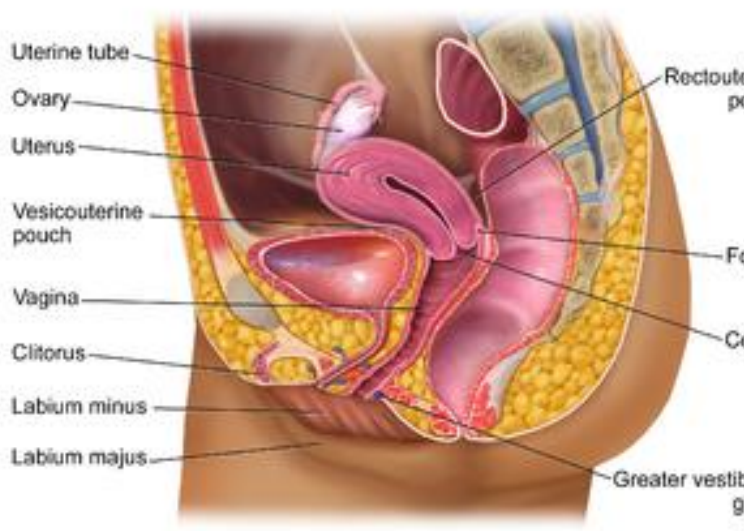

The Female Reproductive System
WikiJournal of Medicine, 2014, 1(2) doi: $10.15347 / \mathrm{wjm} / 2014.010$

Figure Article

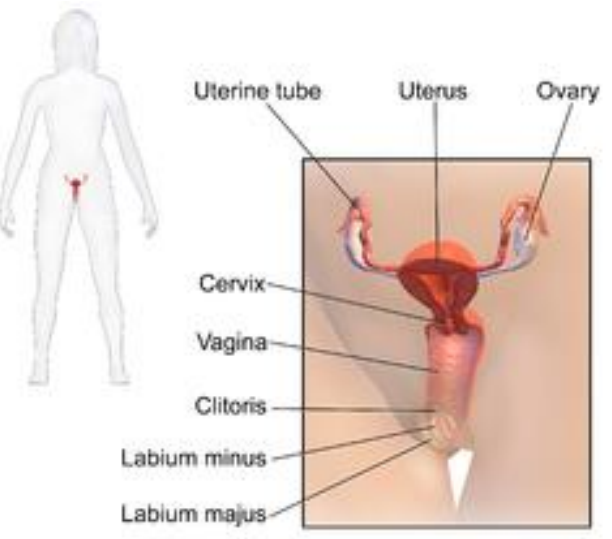

The Female Reproductive System

Female reproductive system.

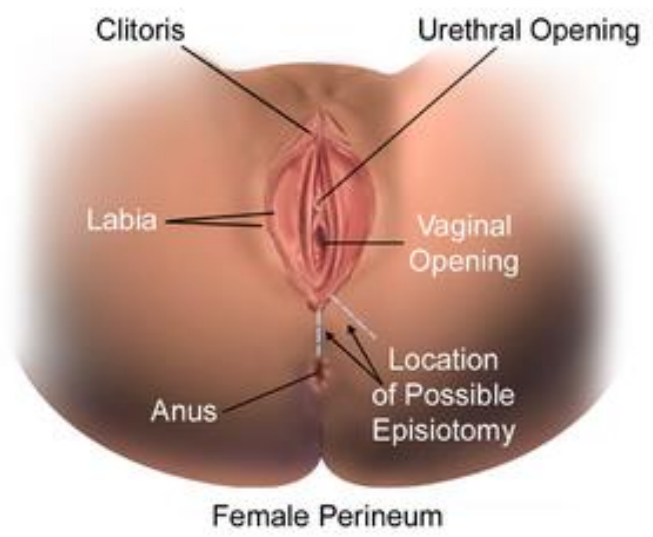

Episiotomy.

Female reproductive system. 
우

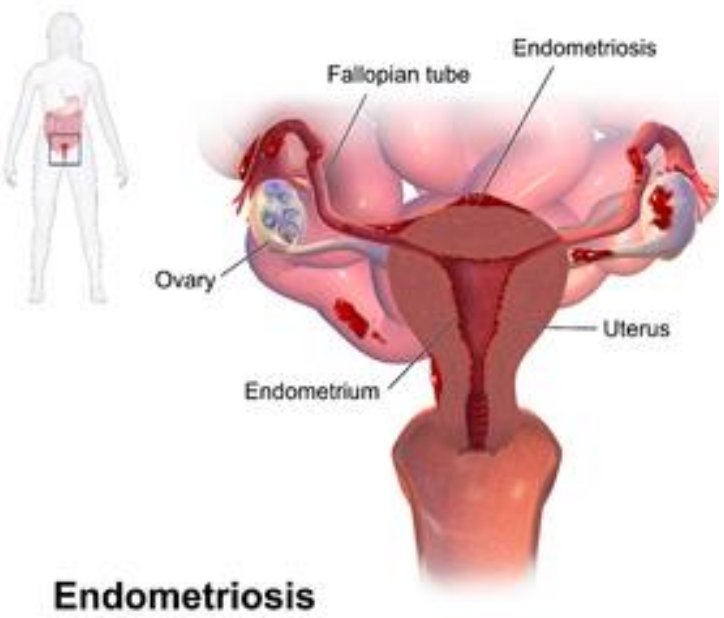

Endometriosis.

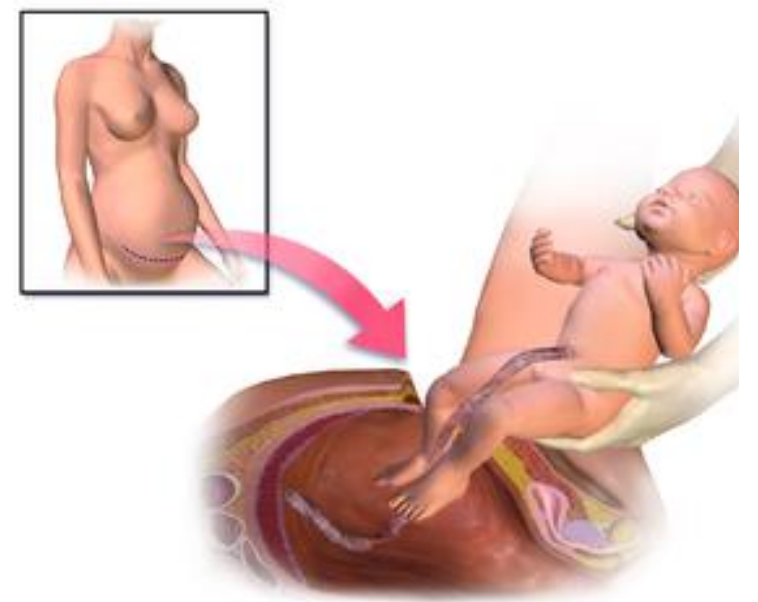

Caesarean section.
WikiJournal of Medicine, 2014, 1(2) doi: $10.15347 / \mathrm{wjm} / 2014.010$

Figure Article

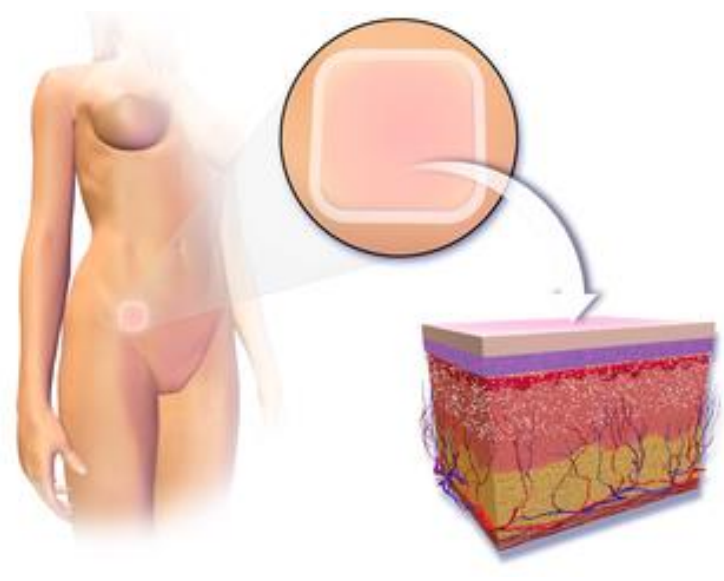

Contraceptive patch.

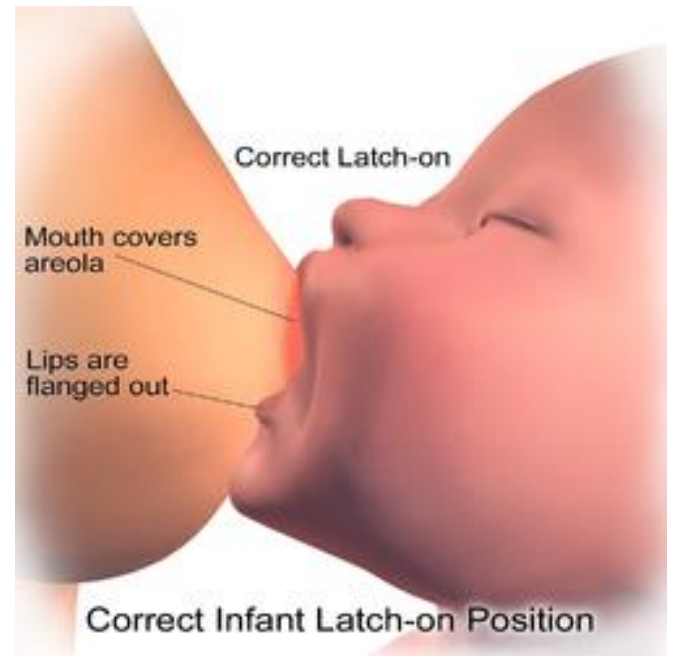

Correct latch-on in breastfeeding. 
Dermatology

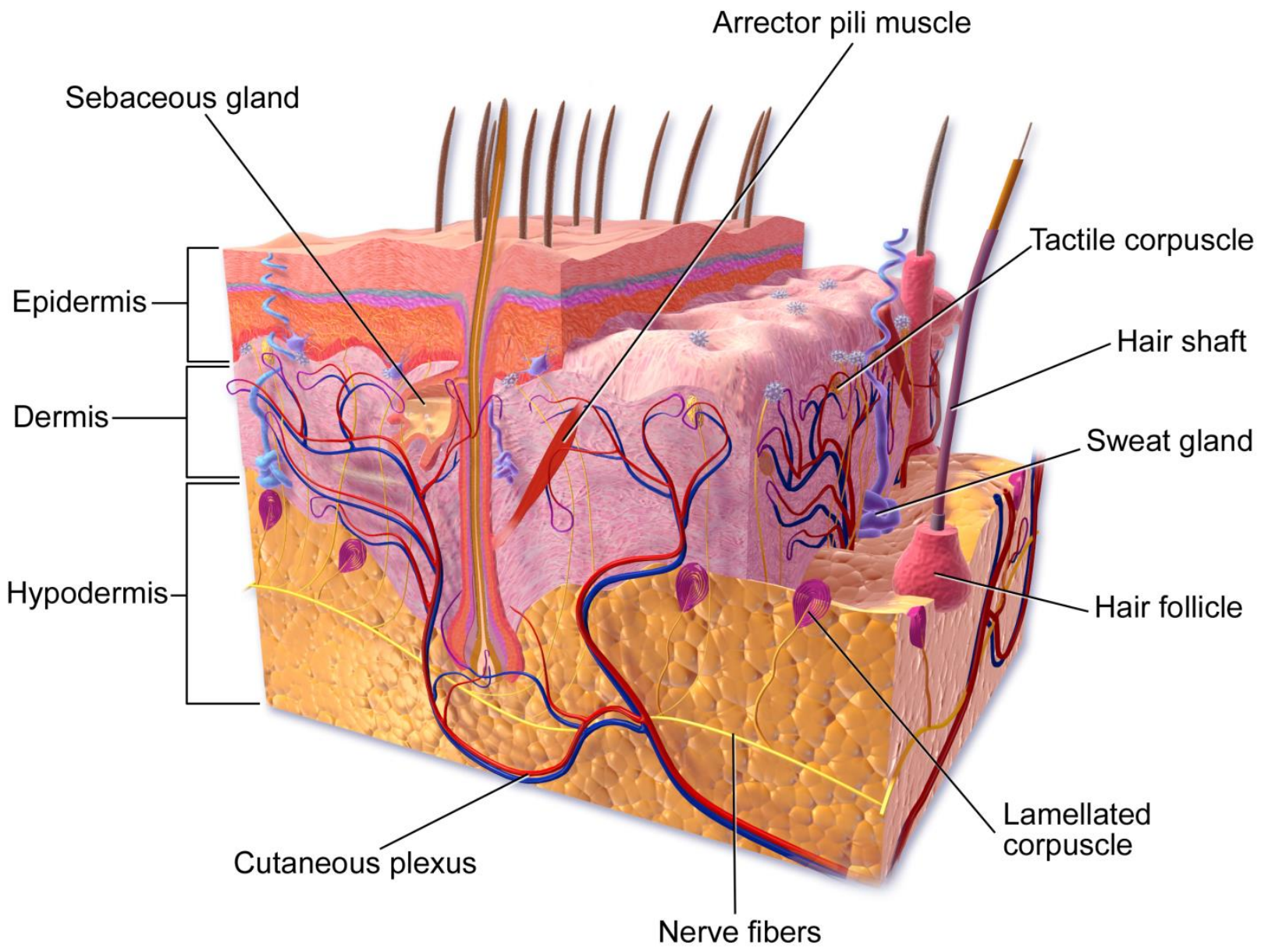

\section{The Components of the Integumentary System}

Anatomy of the skin. 

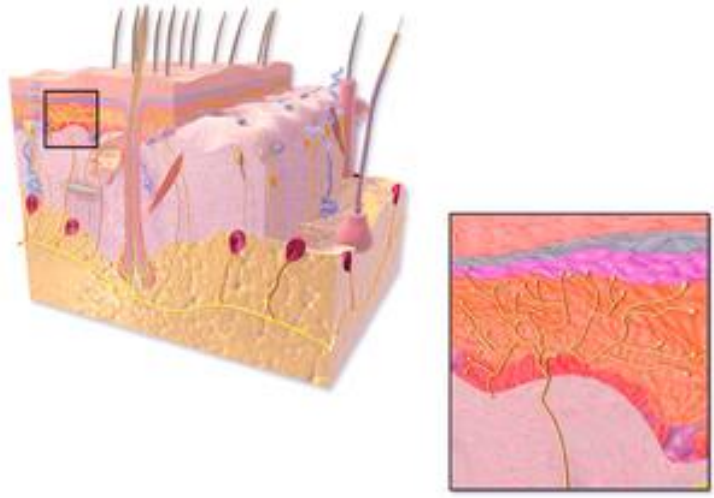

\section{Free Nerve Endings}

Free nerve endings of the skin.

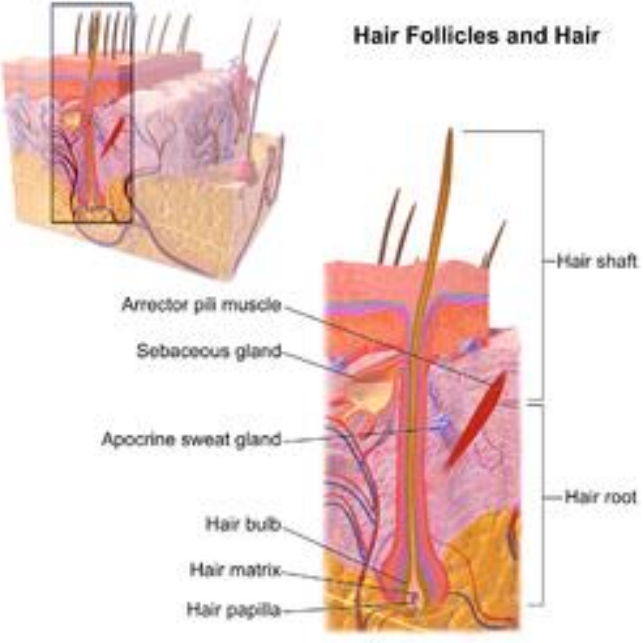

Hair follicle.

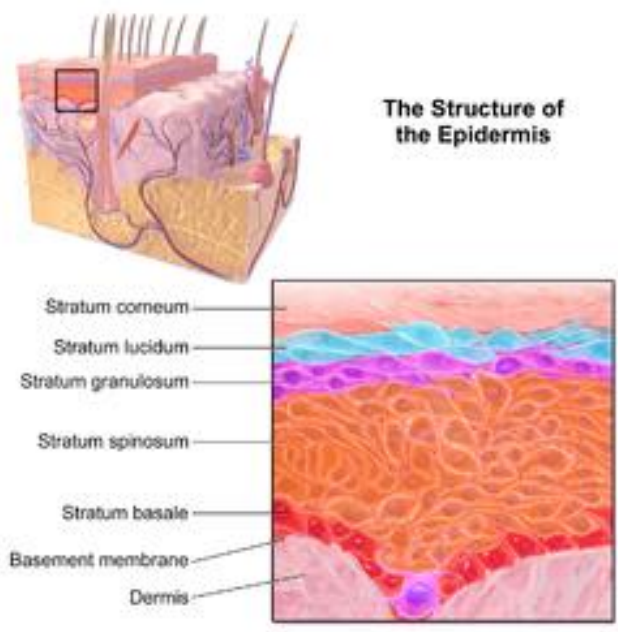

Epidermis.

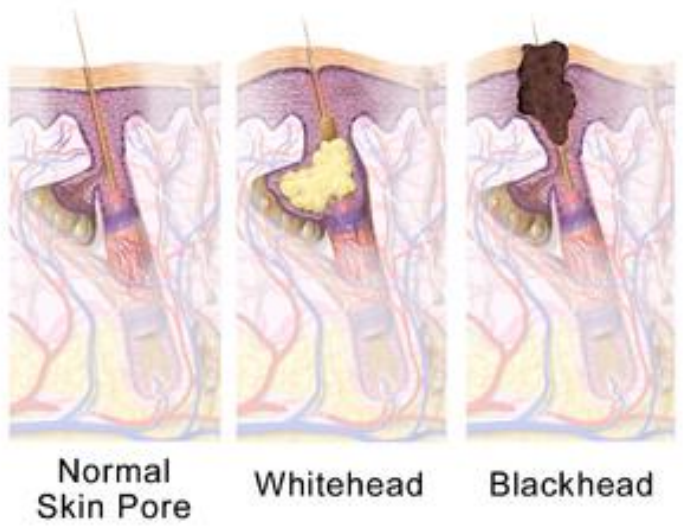

Sebaceous gland states. 

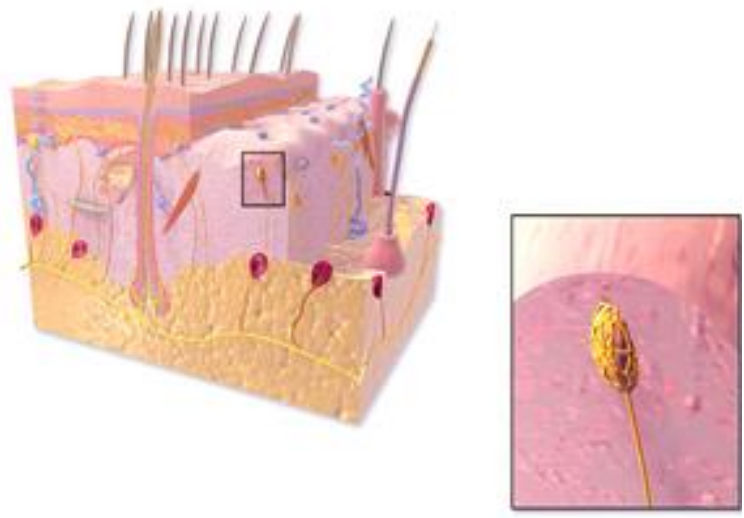

\section{Tactile Corpuscle}

(Meissner's Corpuscle)

Tactile corpuscle.

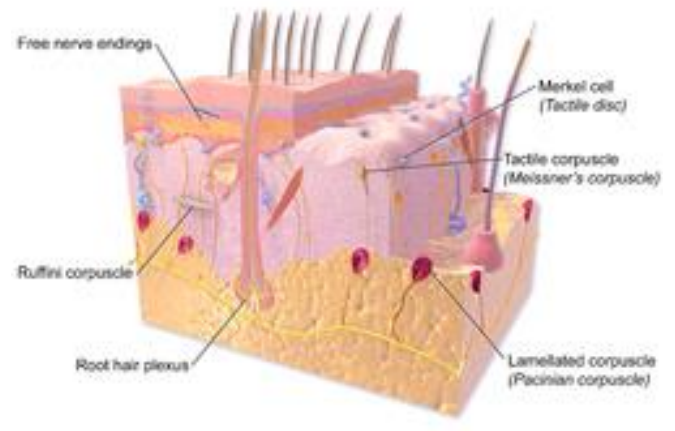

Tactile Receptors in the Skin

Tactile mechanoreceptors of the skin.

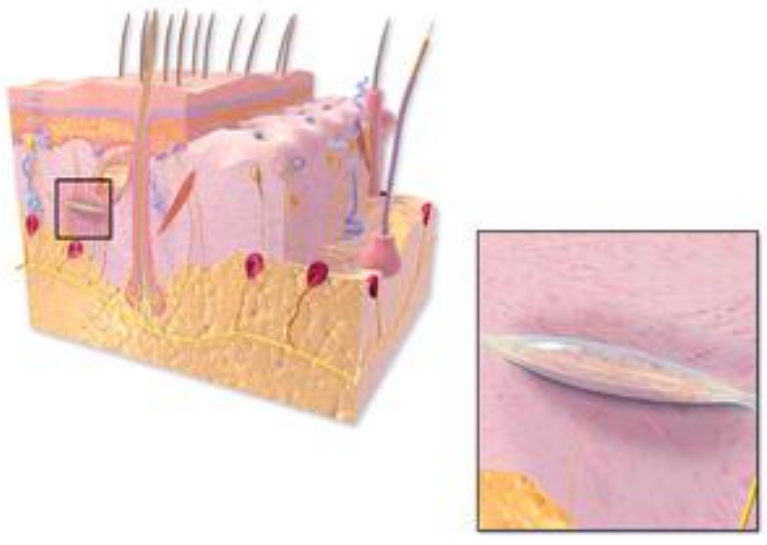

Ruffini Corpuscle

Ruffini corpuscle.

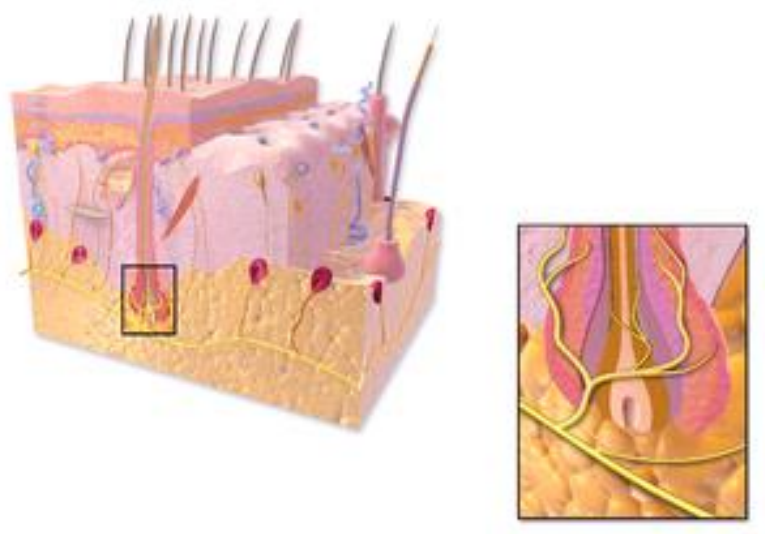

Root Hair Plexus

Hair plexus. 


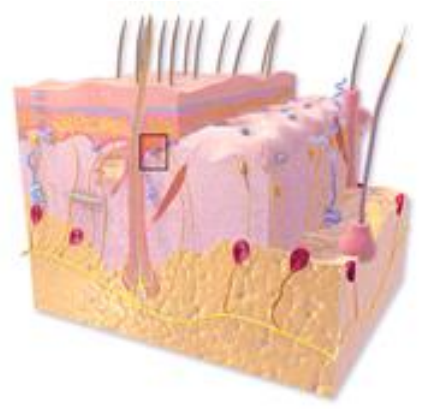

Merkel Cell

(Tactile Disc)
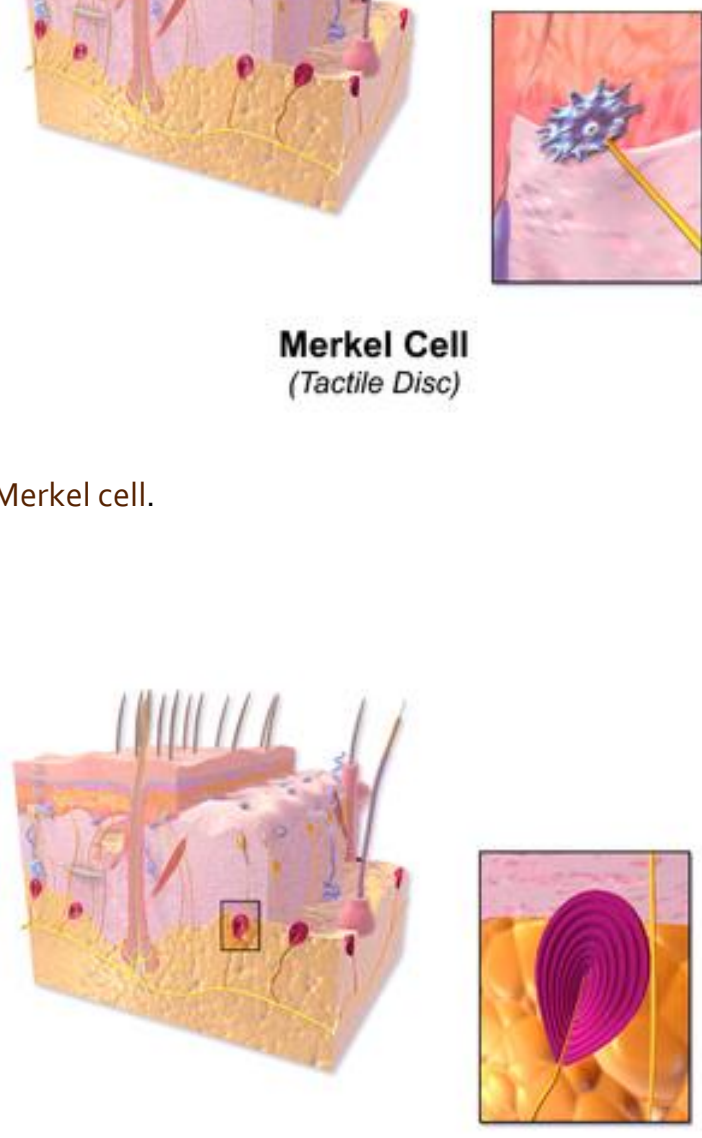

\section{Lamellated Corpuscle (Pacinian Corpuscle)}

Lamellar corpuscle.
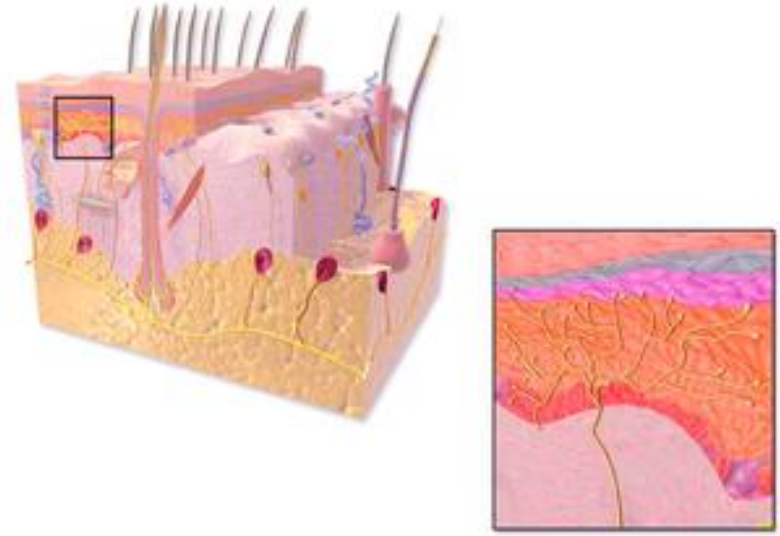

Free Nerve Endings

Free nerve endings.
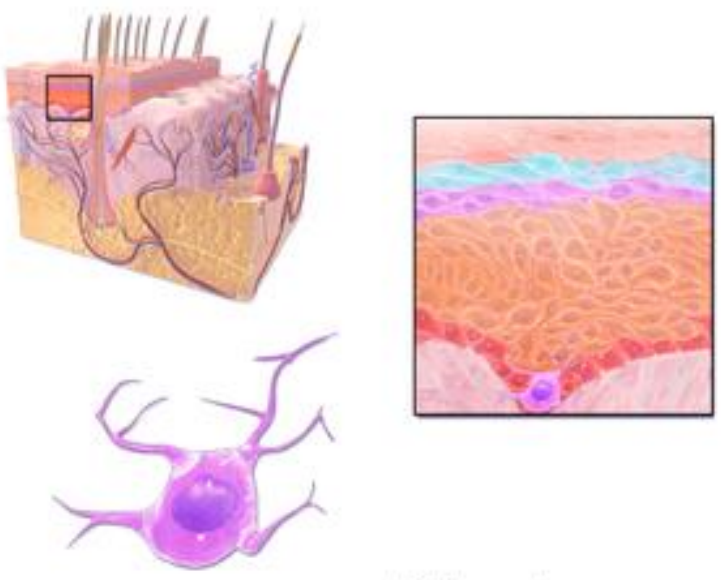

Melanocyte

Location of the melanocyte. 


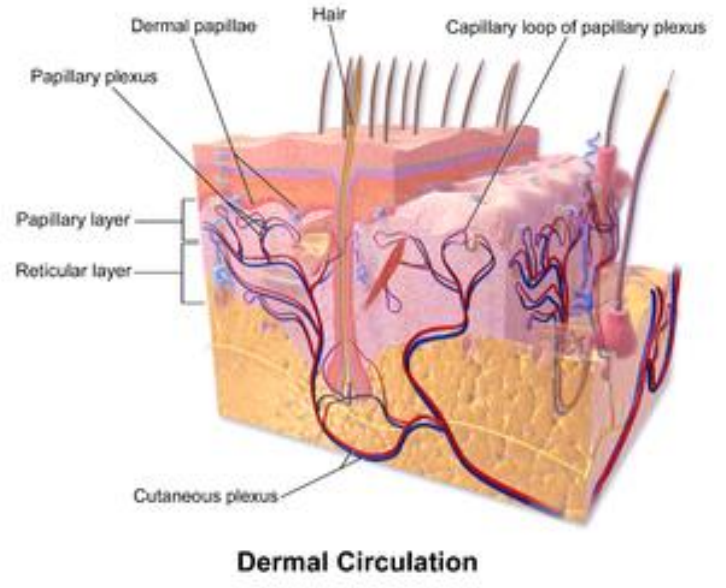

Blood supply of the dermis.

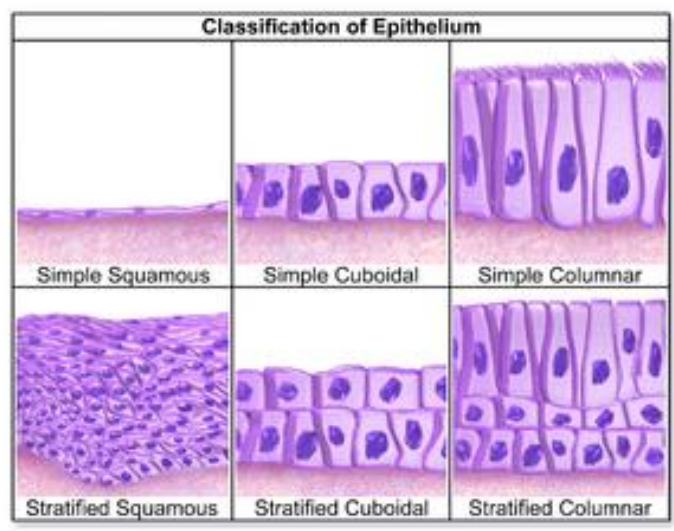

Epithelium classification.

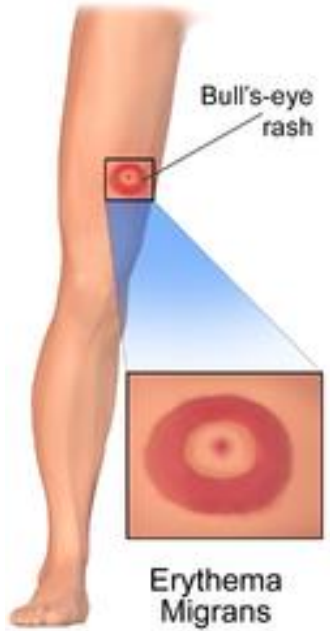

Rash of Lyme disease.

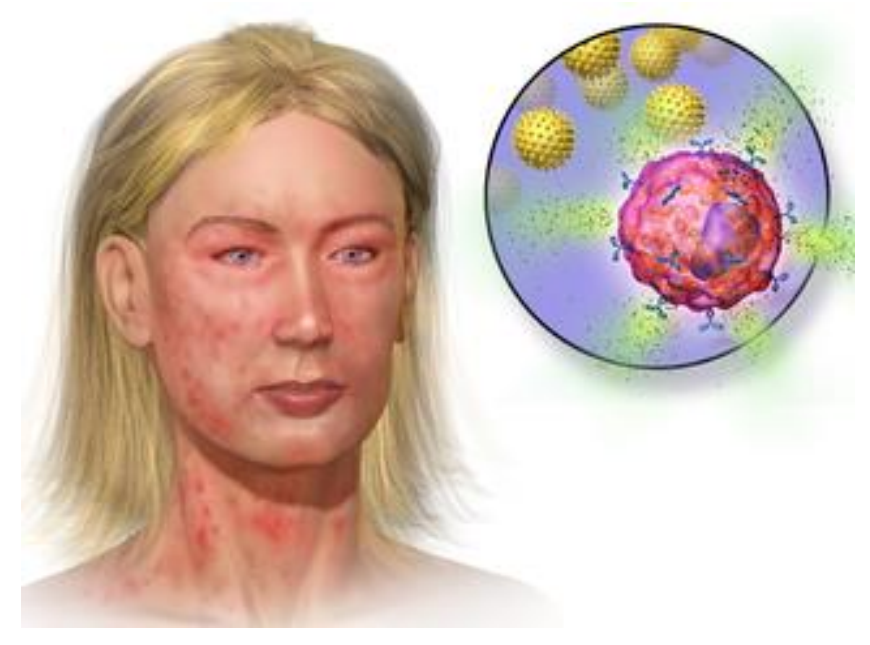

Anaphylaxis. 


\section{Endocrine}

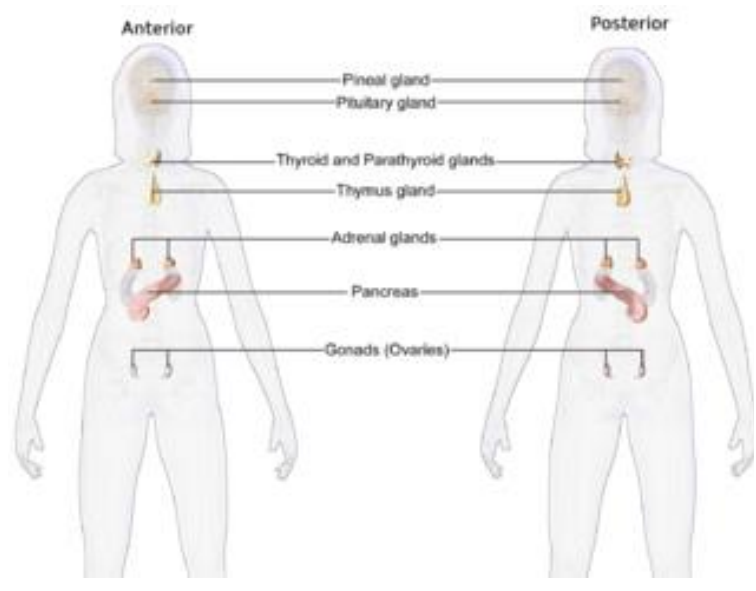

Endocrine system in the female.

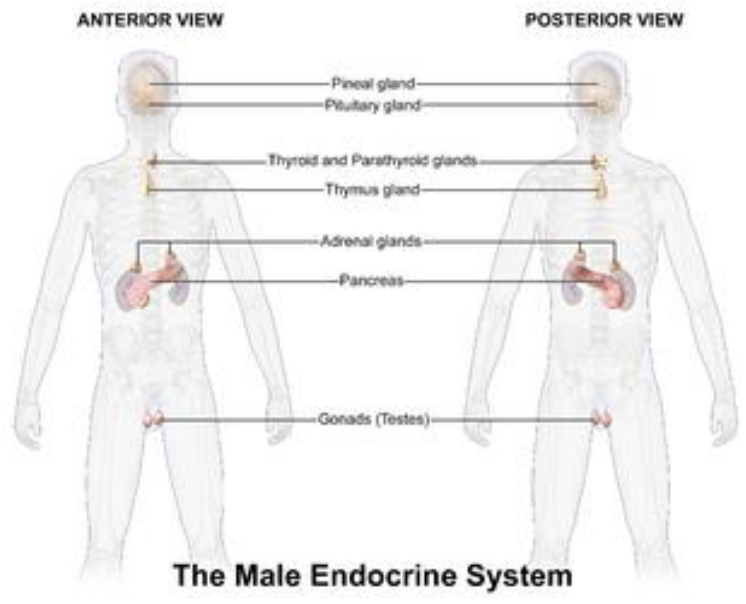

Endocrine system in the male

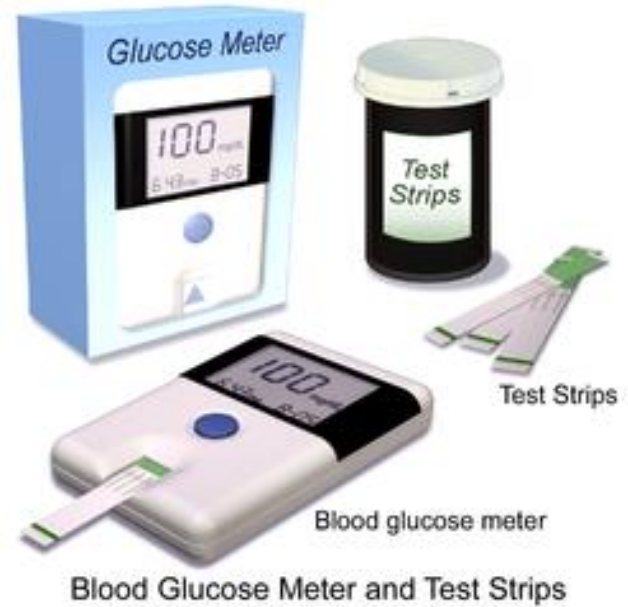

Blood glucose meter.

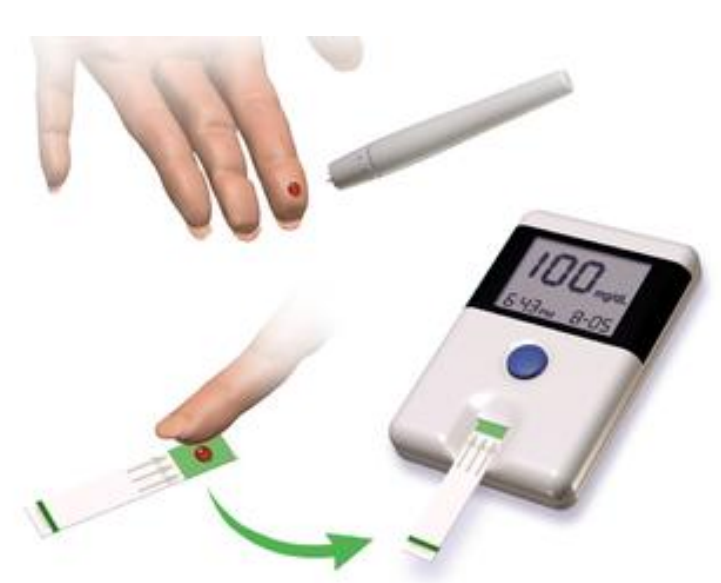

Usage of a blood glucose meter

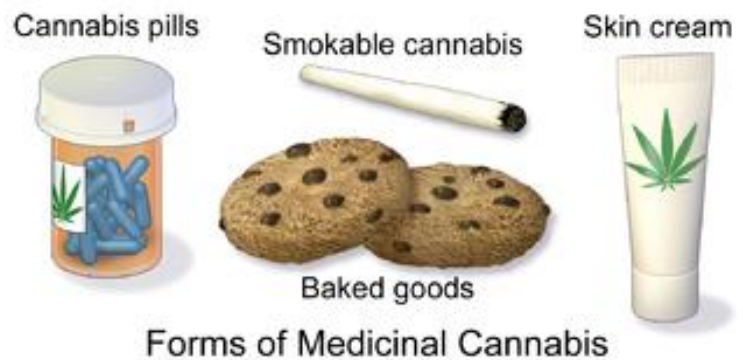

Forms of medical cannabis. 


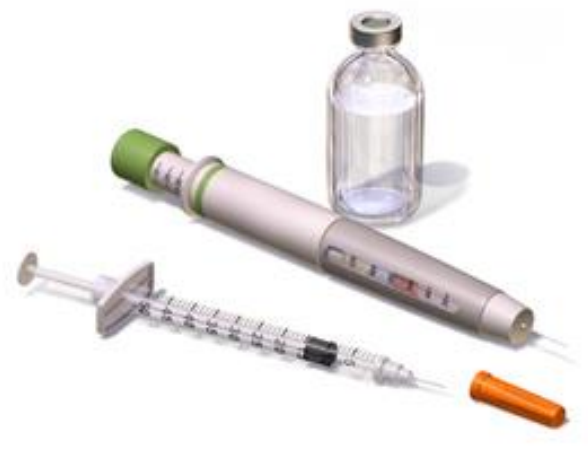

Insulin pen and syringe.

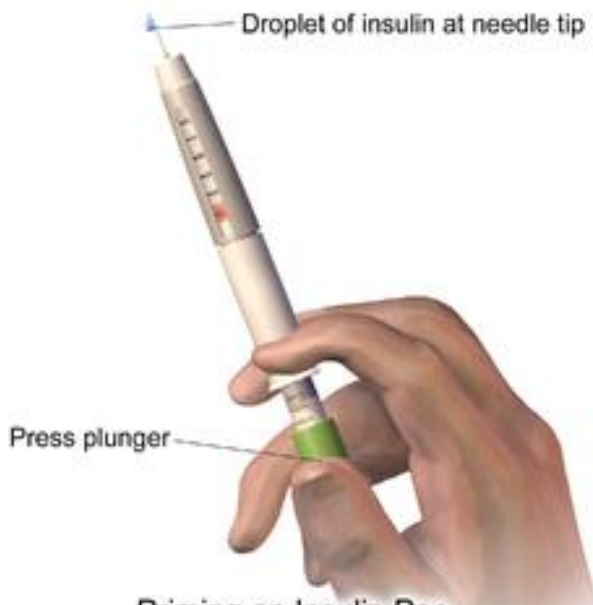

Priming an Insulin Pen

Priming an insulin pen.

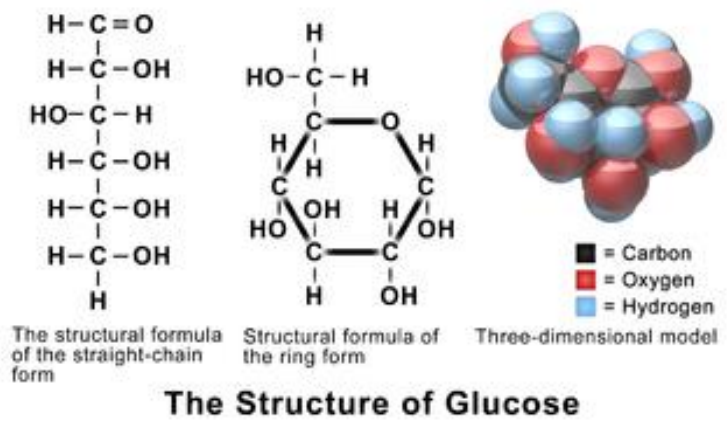

Glucose.

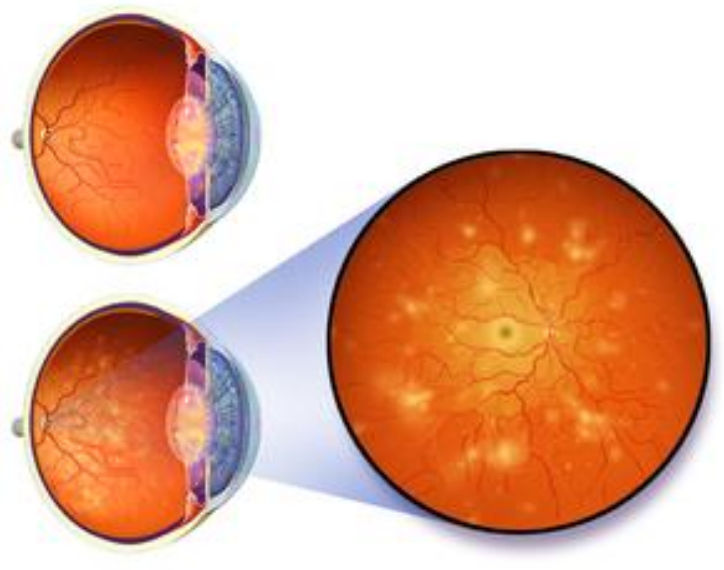

Diabetic retinopathy.

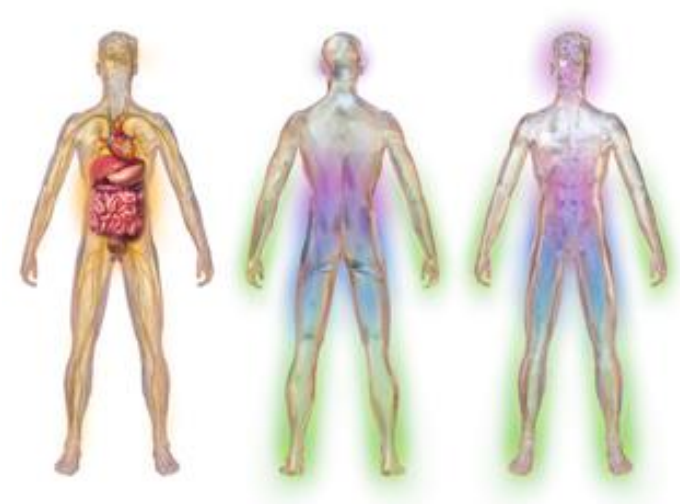

Diabetic neuropathy.

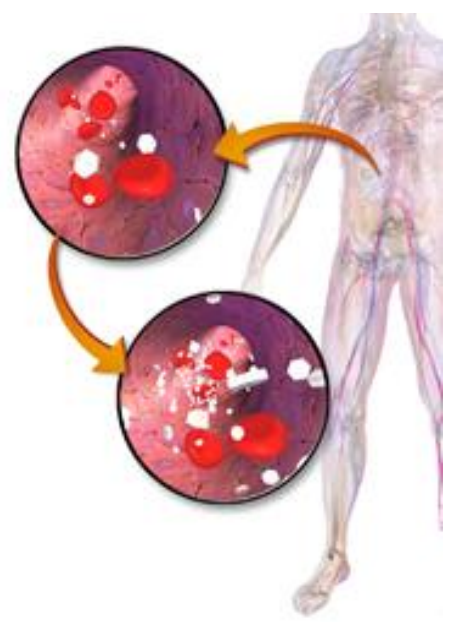

Hyperglycemia. 

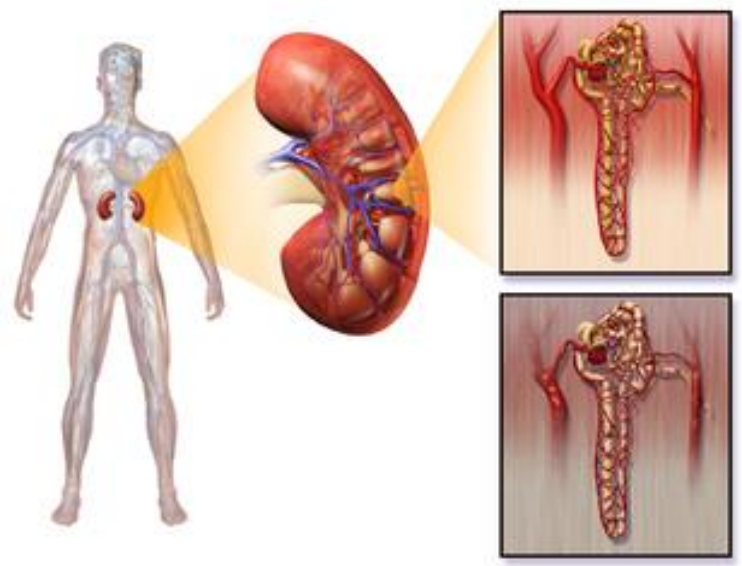

Diabetic nephropathy.<smiles>CCCCCCCCCCCCCC(=O)O</smiles>

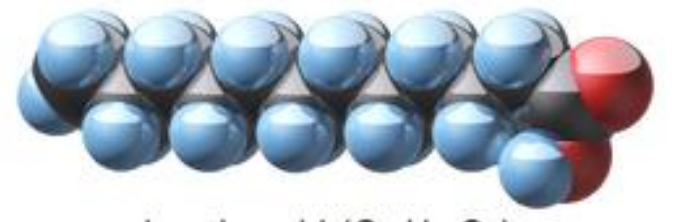

Lauric acid $\left(\mathrm{C}_{12} \mathrm{H}_{24} \mathrm{O}_{2}\right)$

Fatty Acids

Lauric acid, a fatty acid.

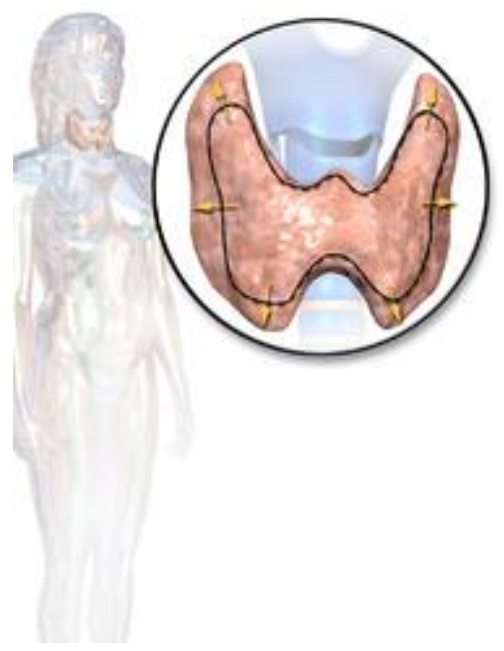

Goiter.

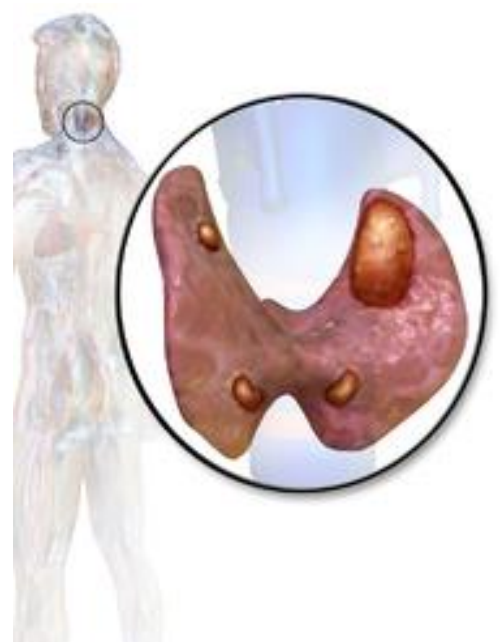

Parathyroid adenoma. 


\section{Respiratory}

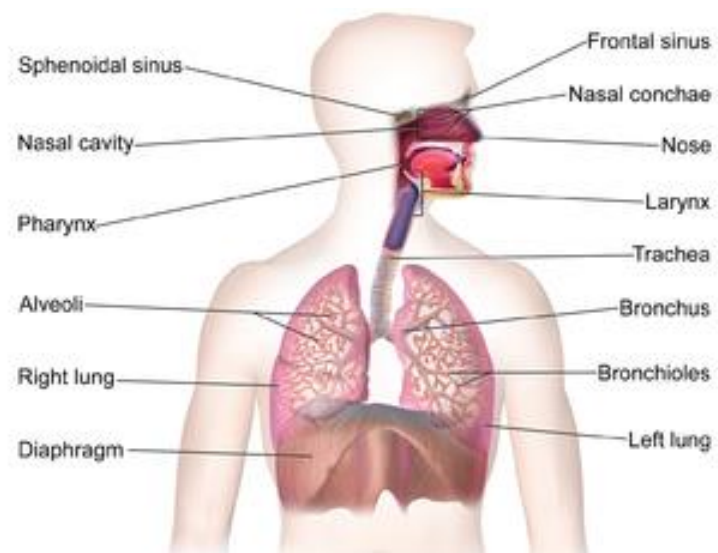

The Respiratory System

Respiratory system.

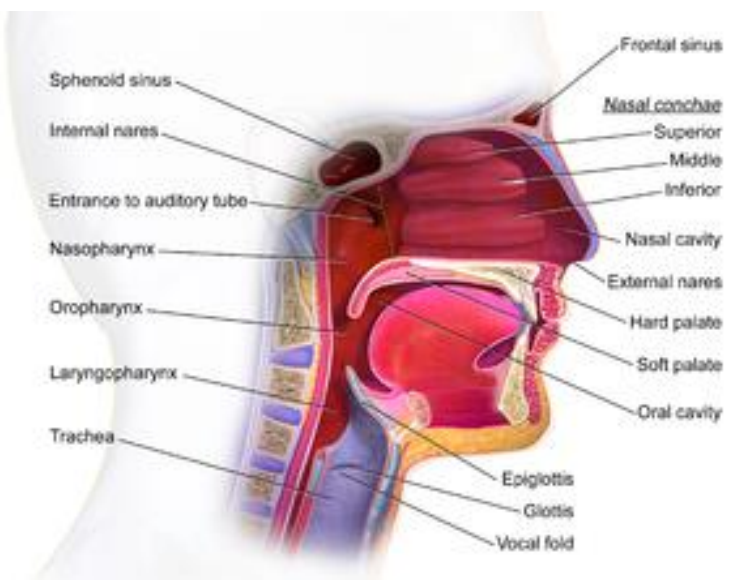

The Upper Respiratory System

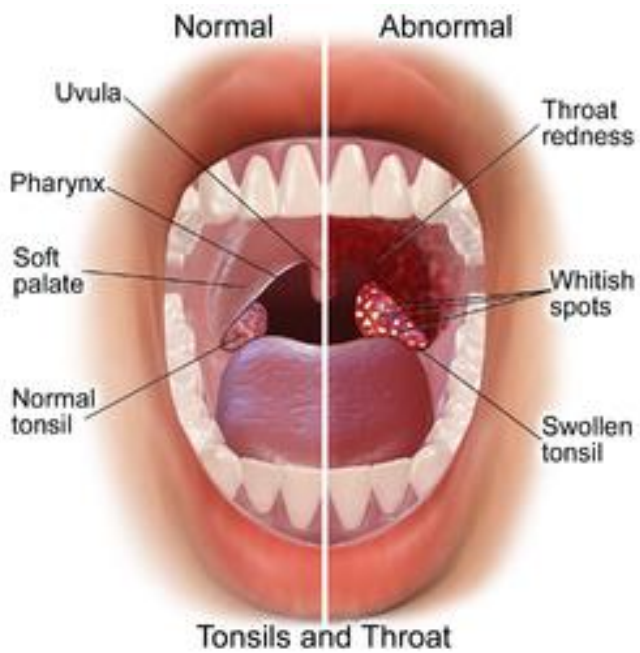

Anatomy of the tonsils and throat.

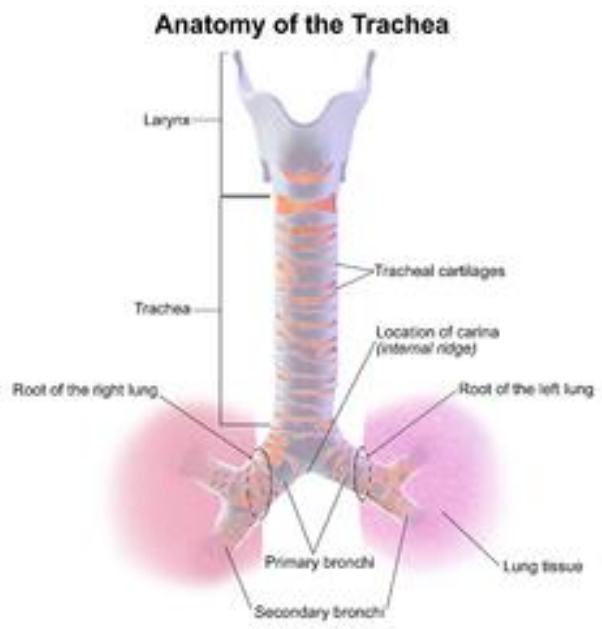

Anatomy of the trachea. 


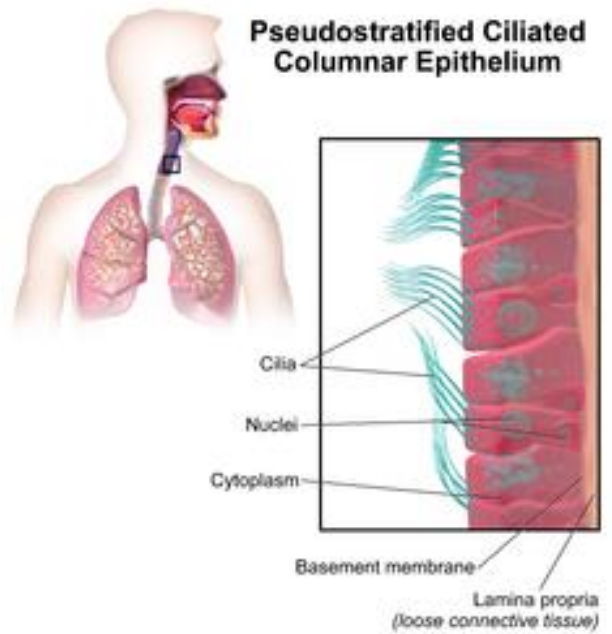

Pseudostratified columnar epithelium in the respiratory tract.

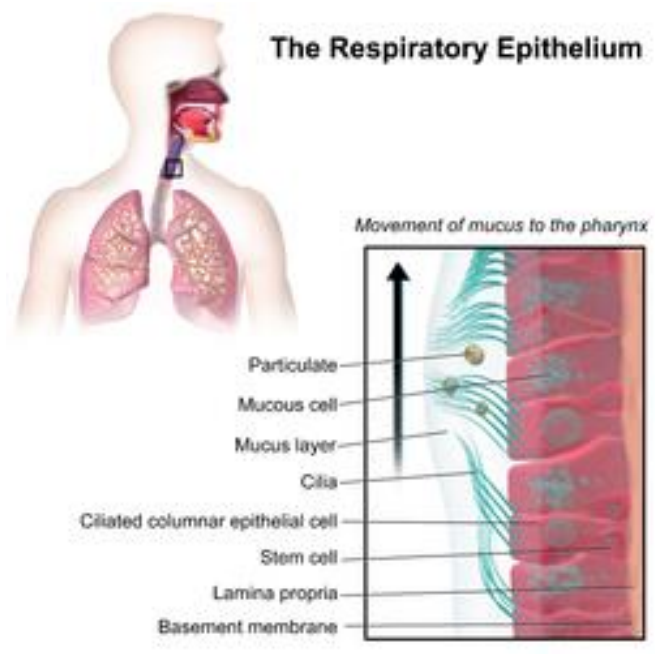

Respiratory epithelium.

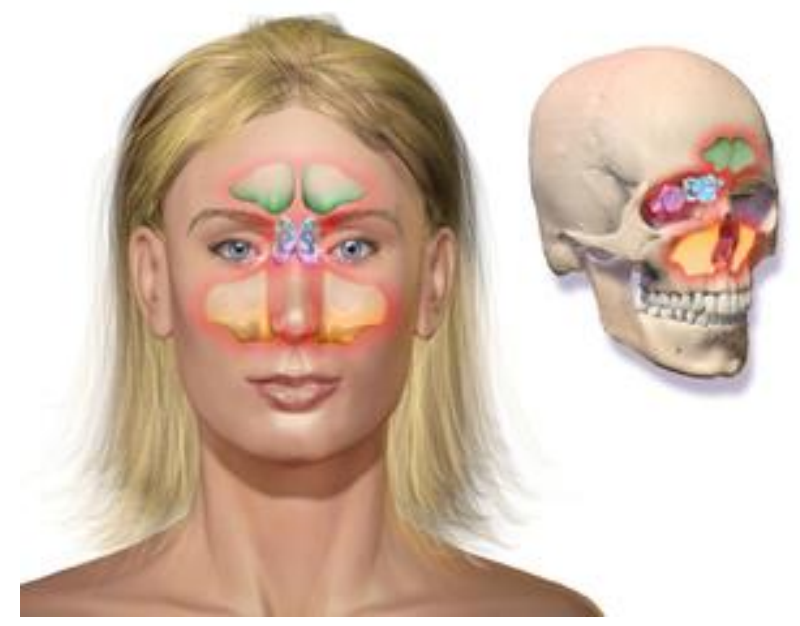

Sinuitis.

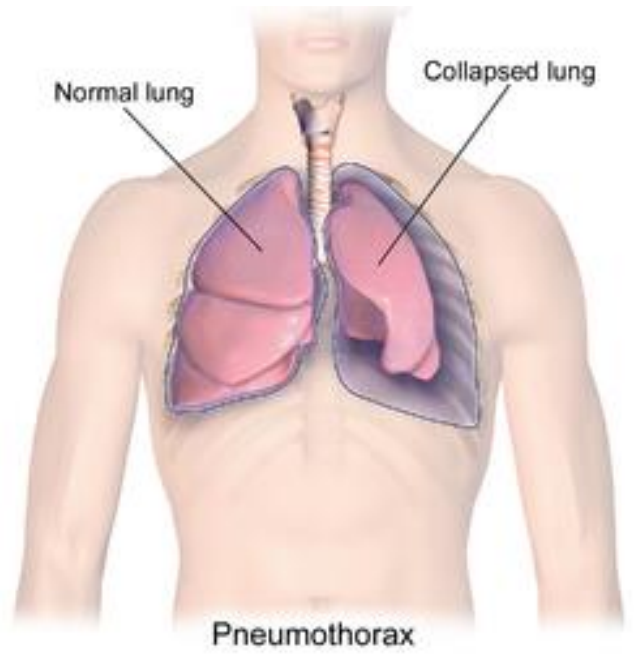

Pneumothorax. 


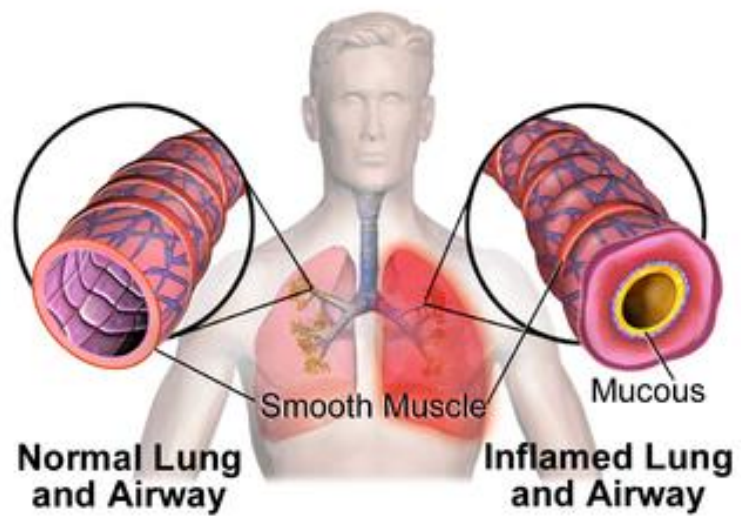

Normal airway versus airway

with bronchoconstriction

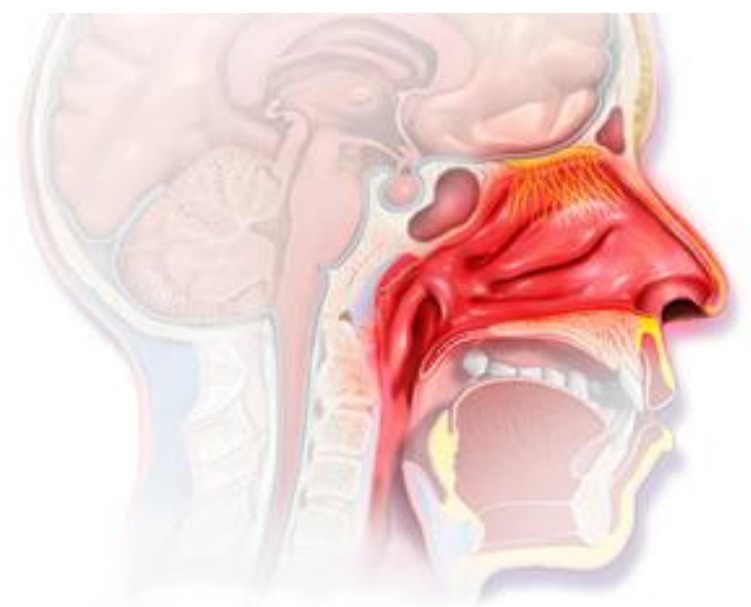

Allergic rhinitis. 


\section{Diseases}

Diseases not primarily belong in any of sections above.

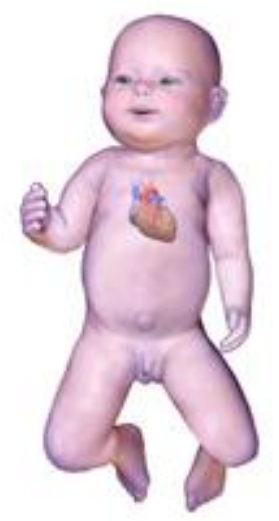

Blue baby syndrome.

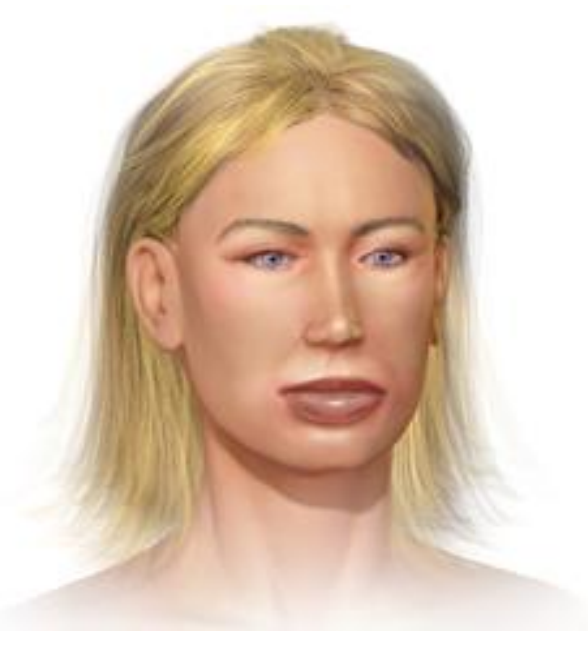

Angioedema.

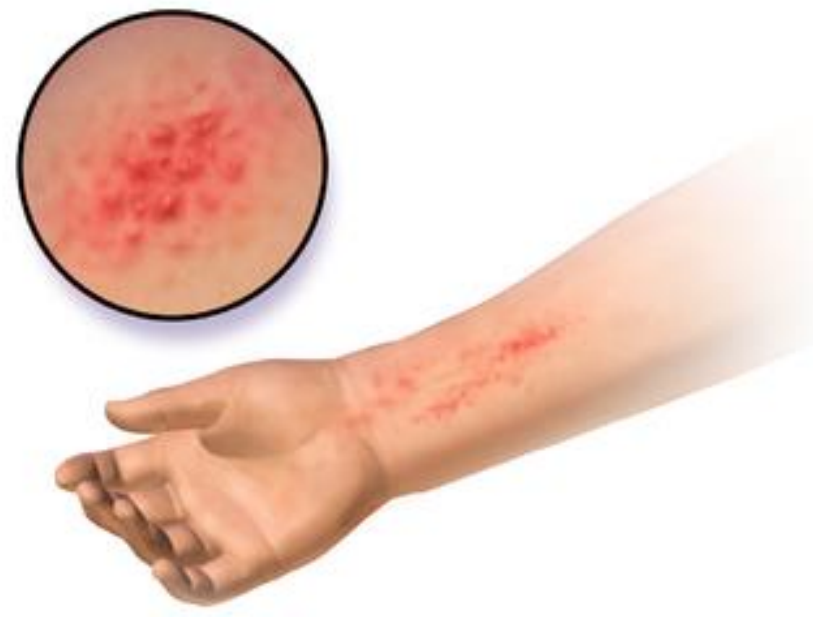

Allergic contact dermatitis

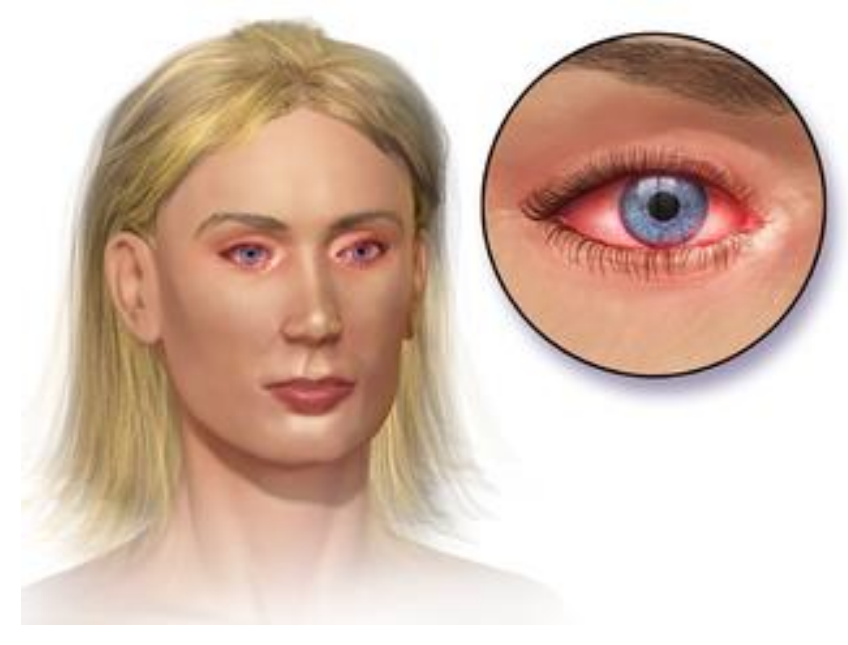

Allergic conjunctivitis. 


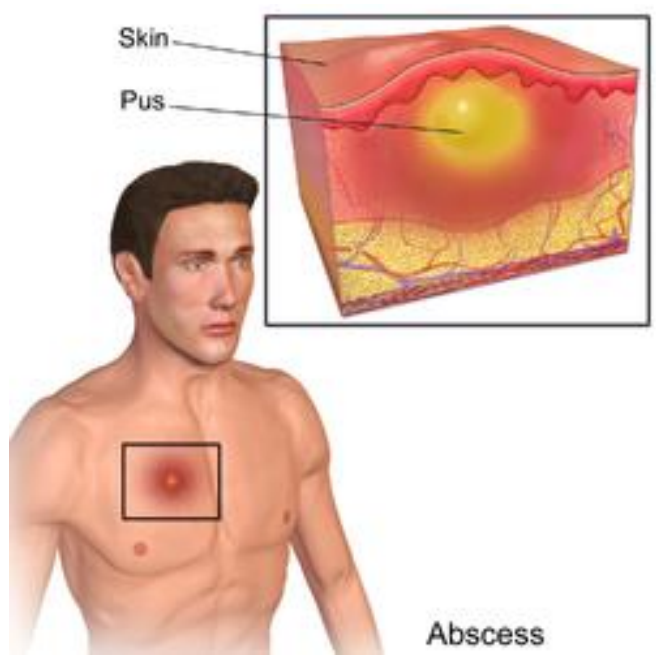

An abscess in the dermis.

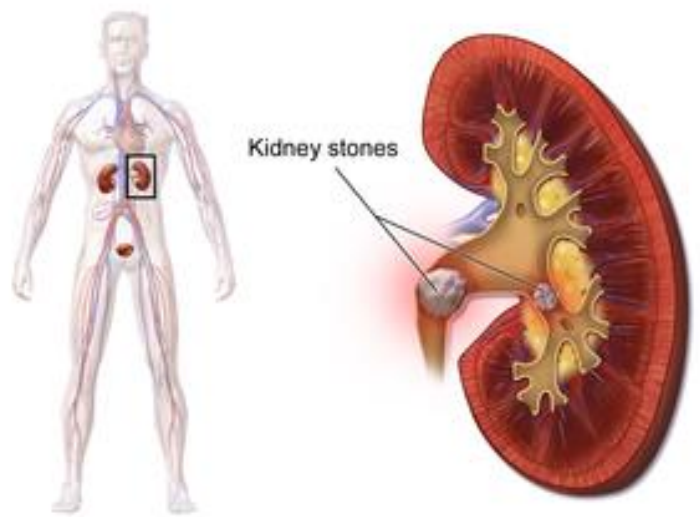

Kidney Stones

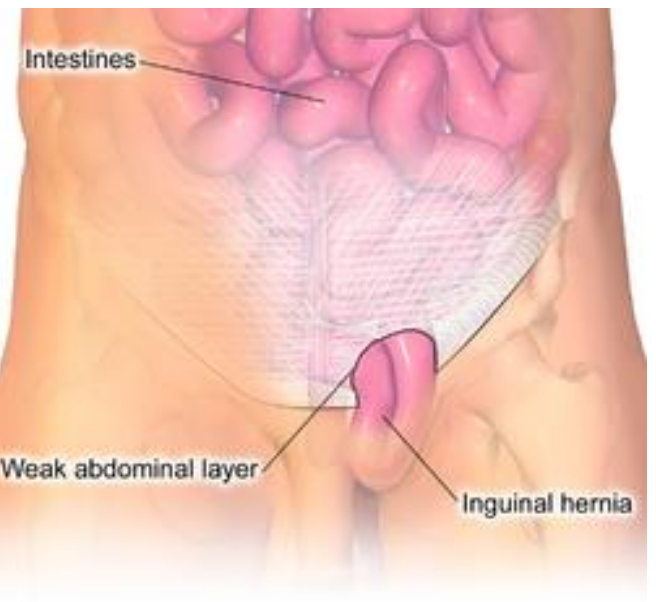

Inguinal Hernia

Inguinal hernia.

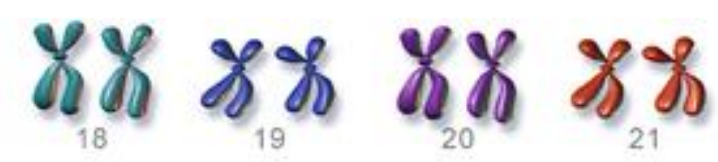

Part of a regular cell showing chromosome 18, 19, 20 , and 21 pairs

\section{ร. \\ 18 \\ 19 \\ 20 \\ 21}

Part of a Down Syndrome child's cell showing chromosome 21 pair with 1 extra chromosome

\section{Down Syndrome}

Karyotyoe in Down syndrome. 


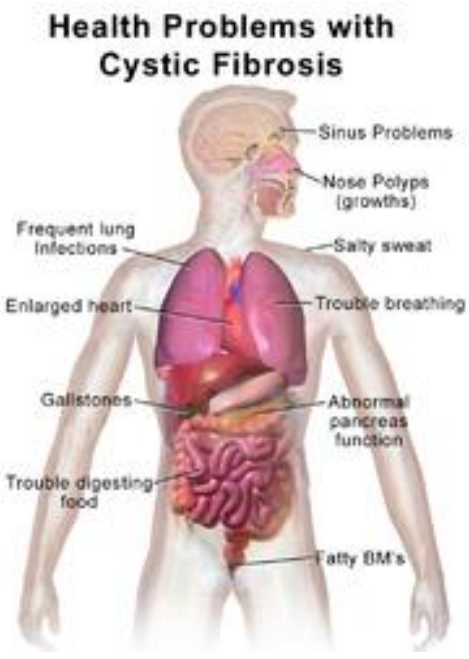

Cystic fibrosis.

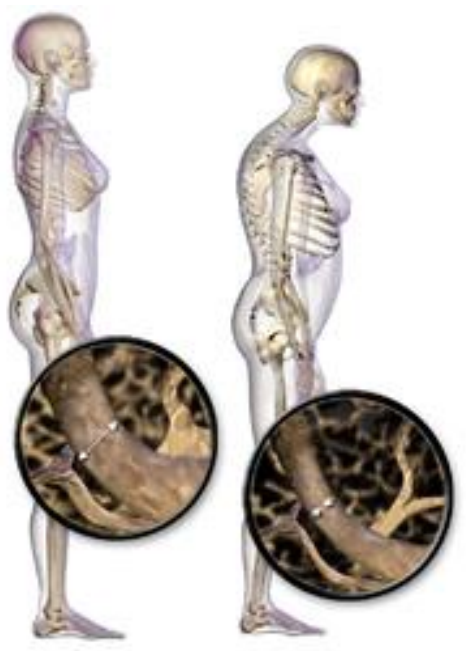

Osteoporosis.

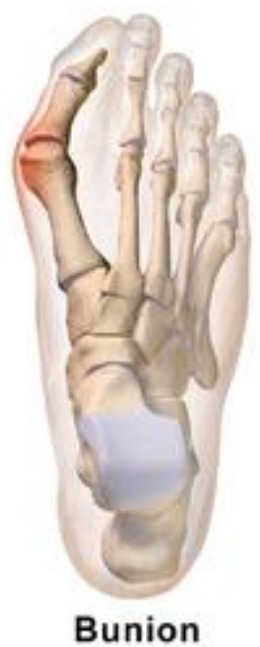

Bunion.

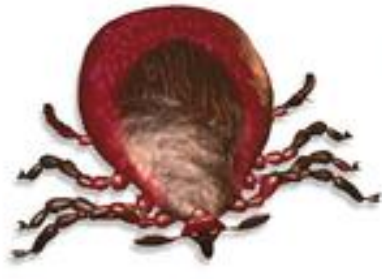

Male

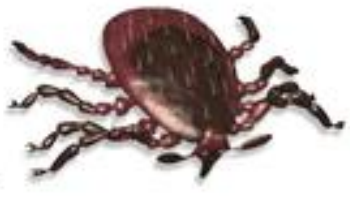

Female

\section{Deer Ticks}

Deer ticks 
Other anatomy

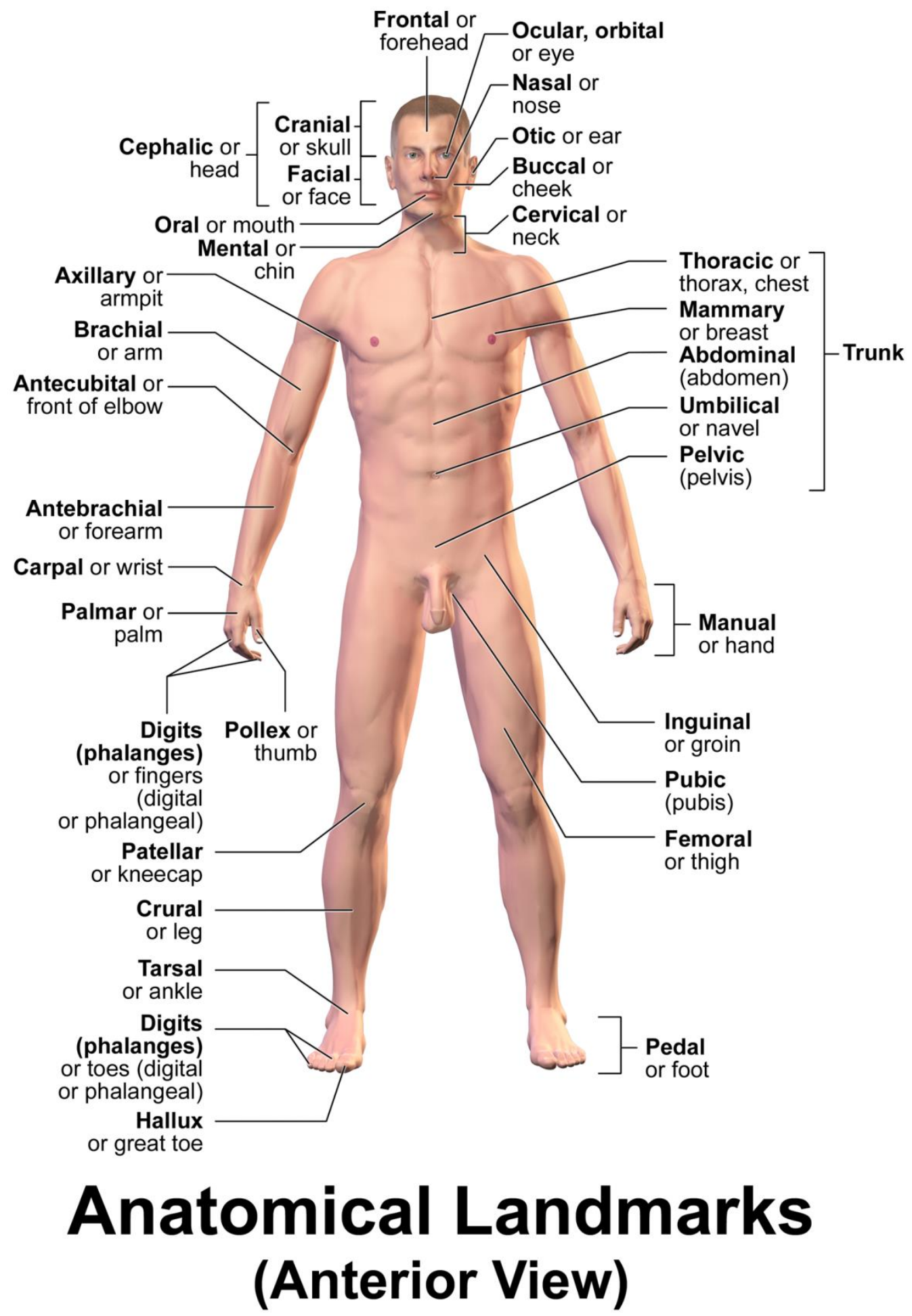

Landmarks of surface anatomy, anterior view. 


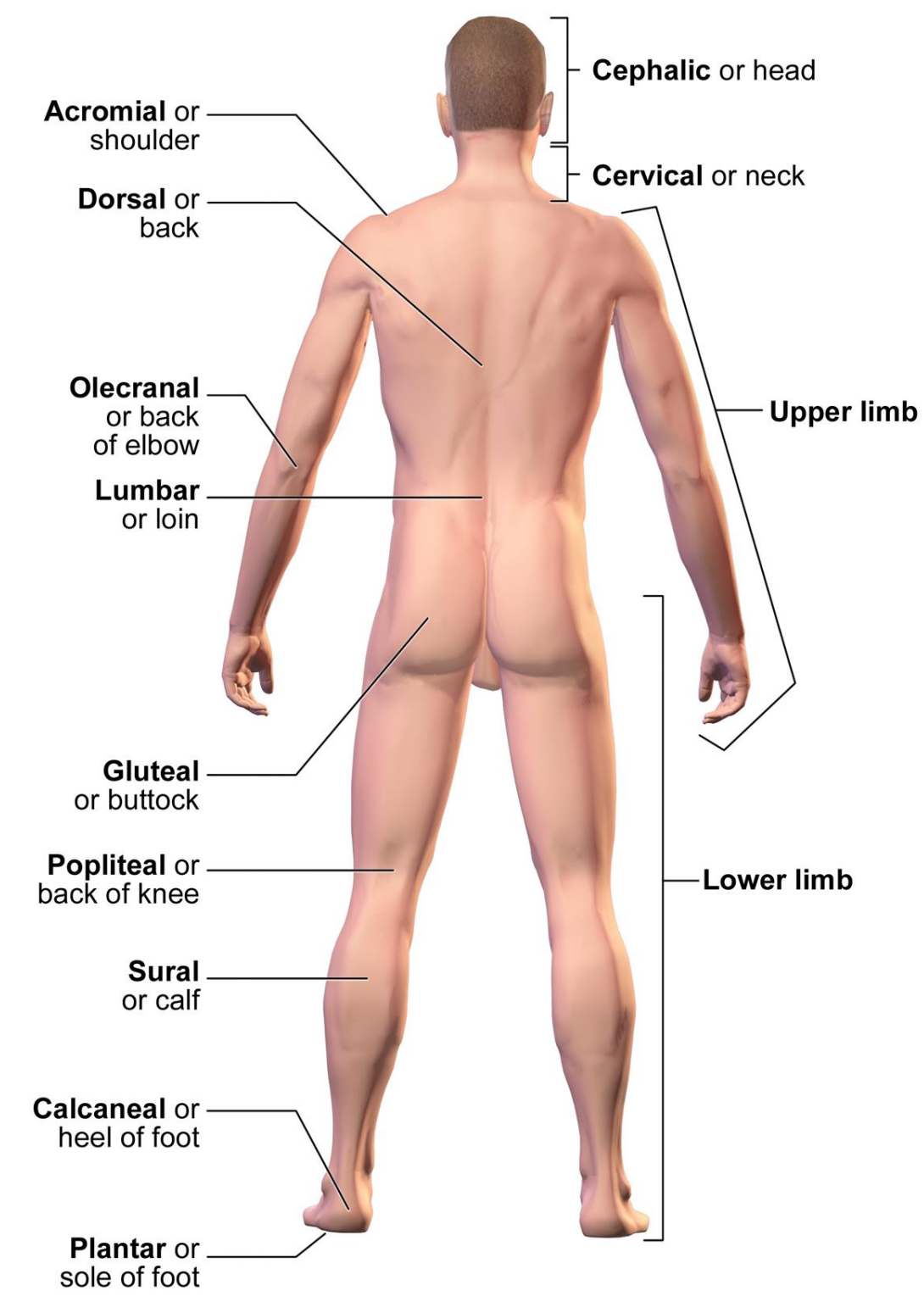

\section{Anatomical Landmarks (Posterior View)}

Landmarks of surface anatomy, posterior view. 

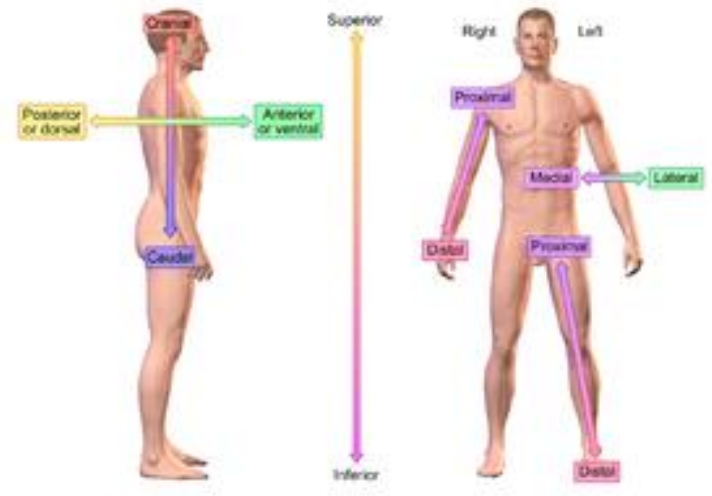

Lateral view
Anterior view

Anatomical terms of location.

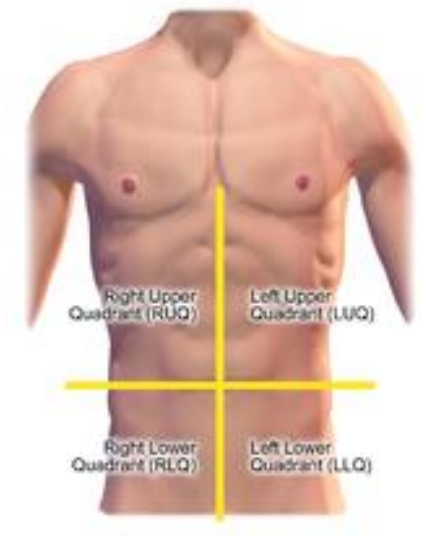

Abdominopelvic Quadrants

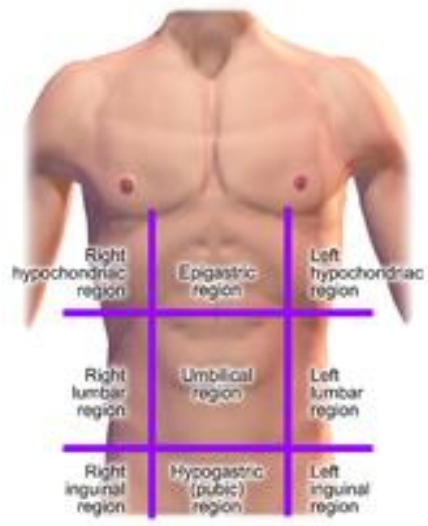

Abdominopelvic Regions

9-region scheme of the abdomen.

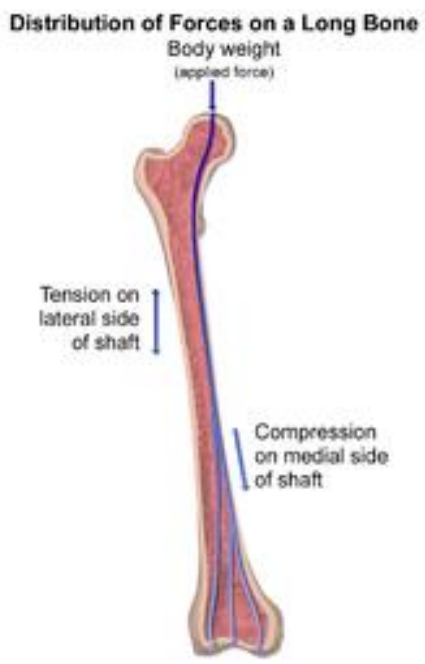

Distribution forces of the femur.

Abdominal quadrants 
(อ) (c)

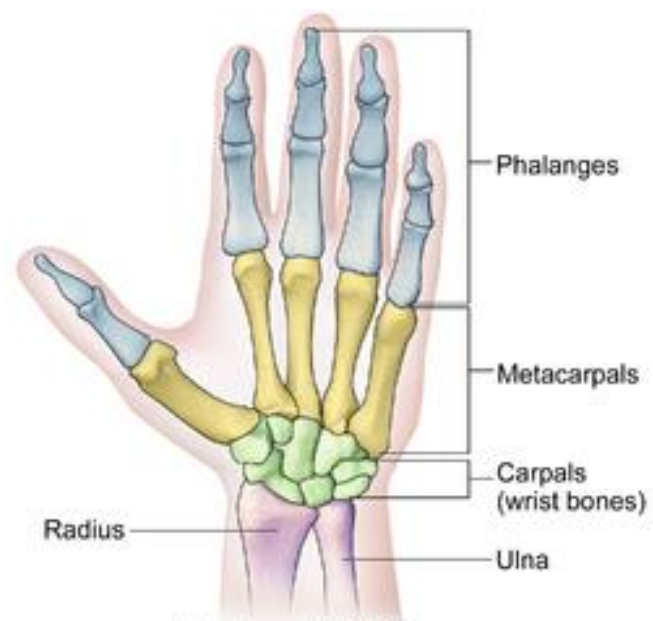

Hand and Wrist Bones

Skeletal anatomys of the hand.

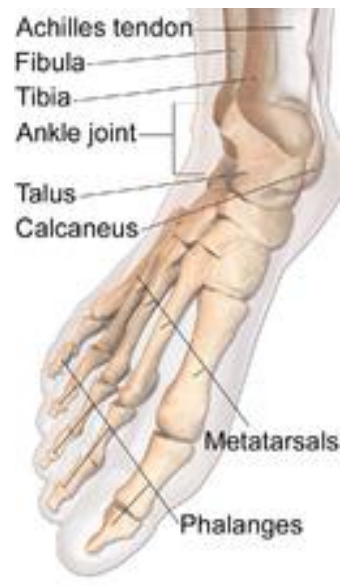

Lower Leg and Foot

Skeletal anatomys of the foot.
WikiJournal of Medicine, 2014, 1(2) doi: $10.15347 / \mathrm{wjm} / 2014.010$

Figure Article
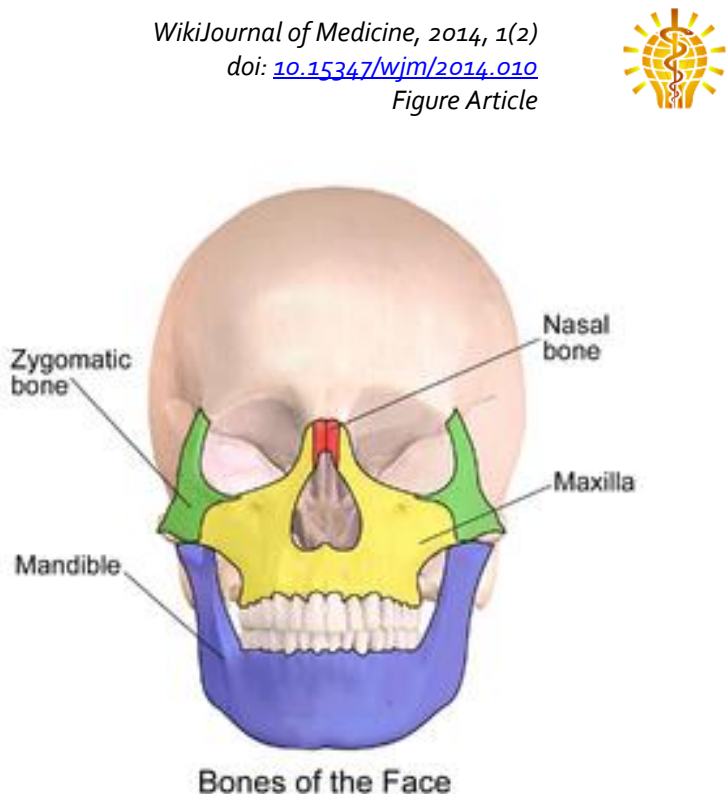

Facial bones

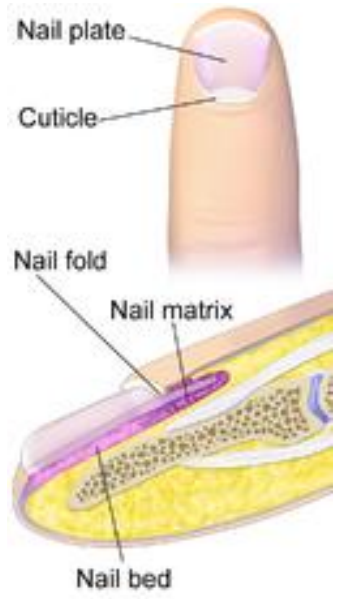

Nail Anatomy

Nail. 
우

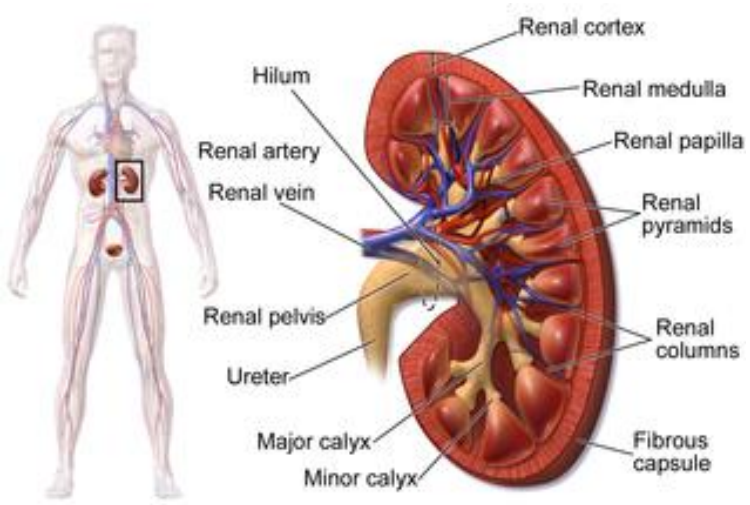

Kidney Anatomy

Kidney anatomy.

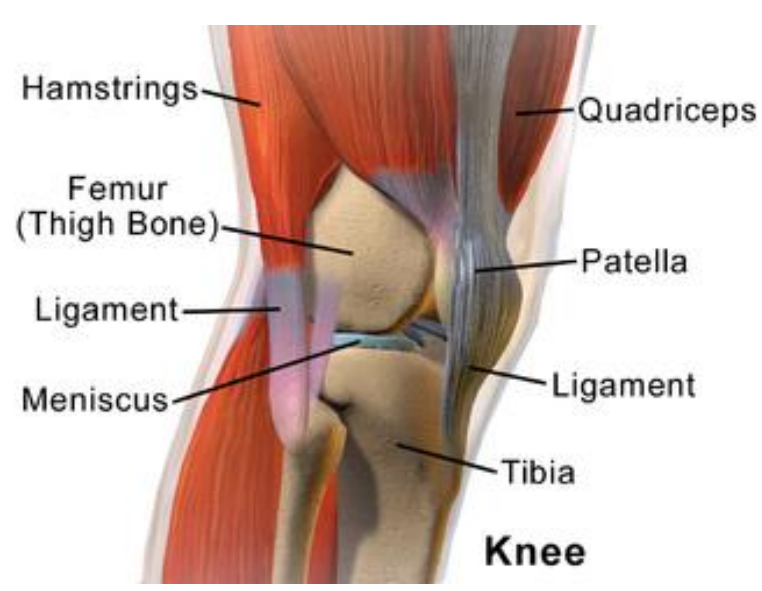

Anatomy of the knee, viewed from the side.
WikiJournal of Medicine, 2014, 1(2) doi: $10.15347 / \mathrm{wjm} / 2014.010$

Figure Article

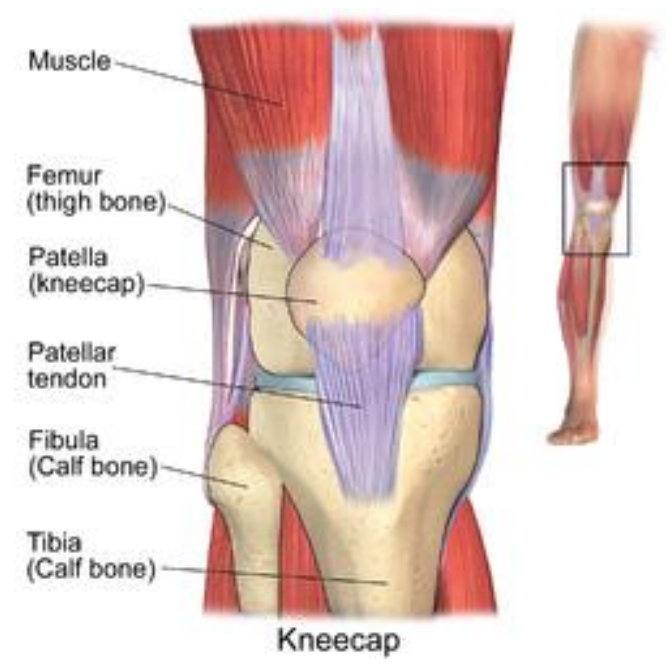

Anatomy of the knee, viewed from front.

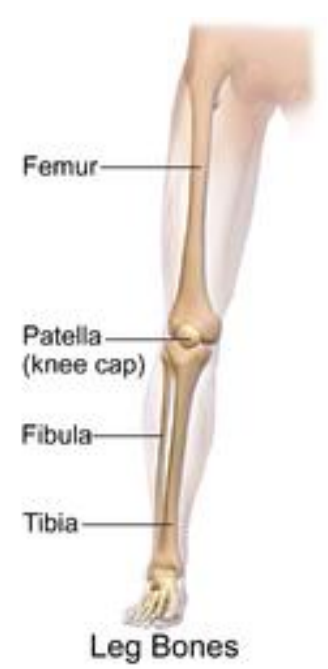

Bones of the leg. 


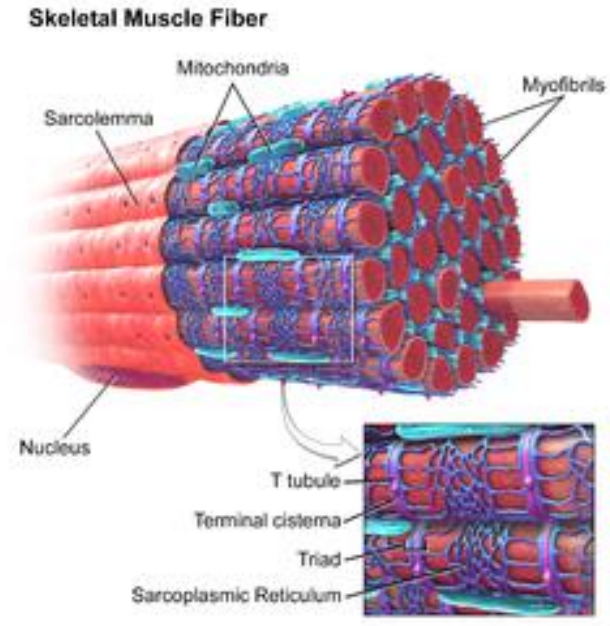

Skeletal muscle.

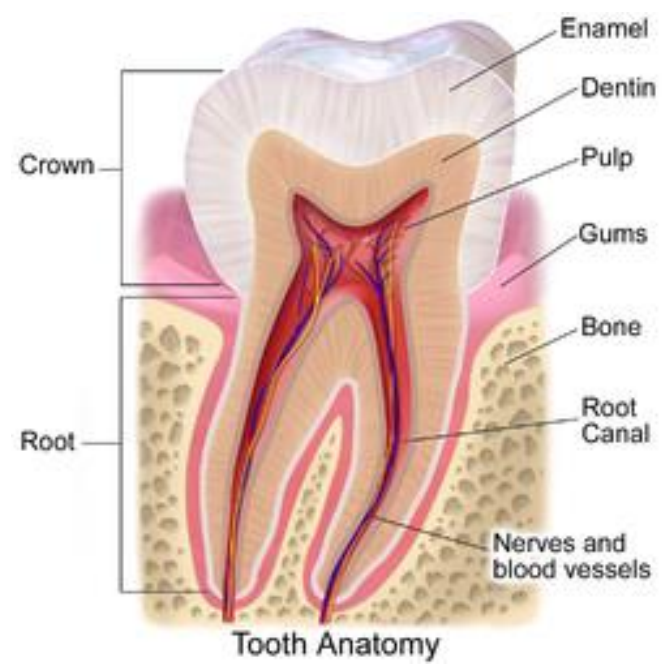

Tooth anatomy

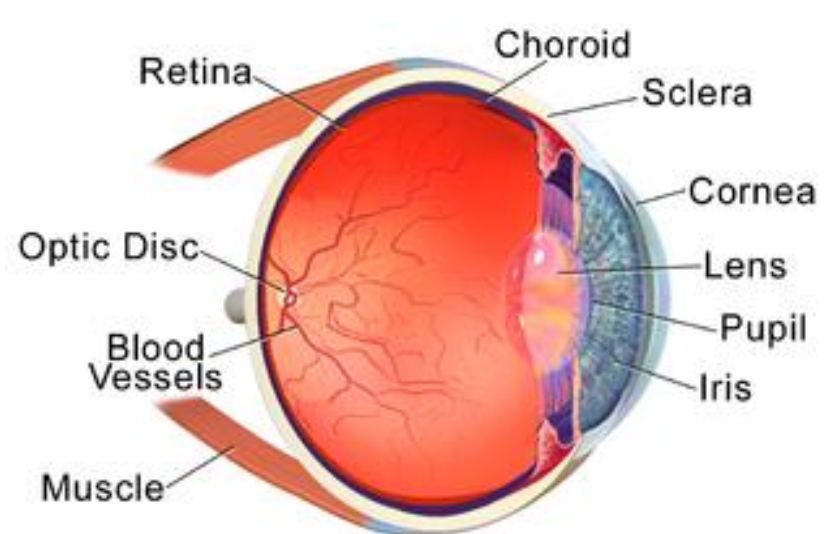

\section{Anatomy of the Eye}

Eye.

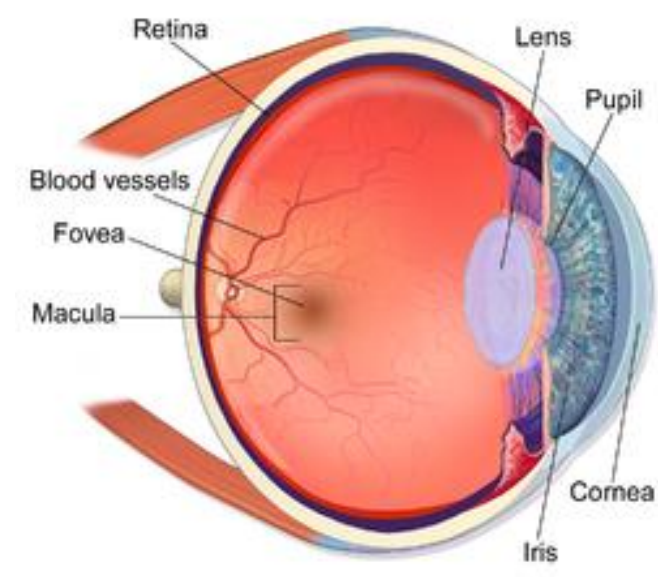

Eye Anatomy

Eye. 


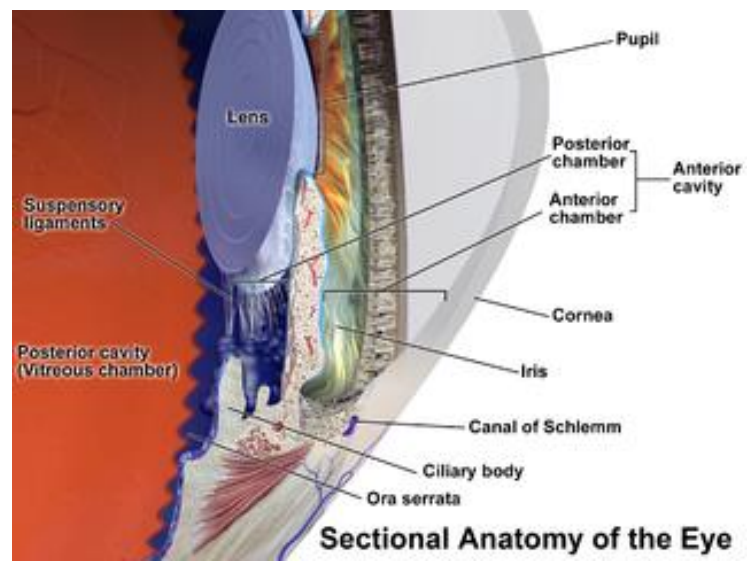

Anterior eye.

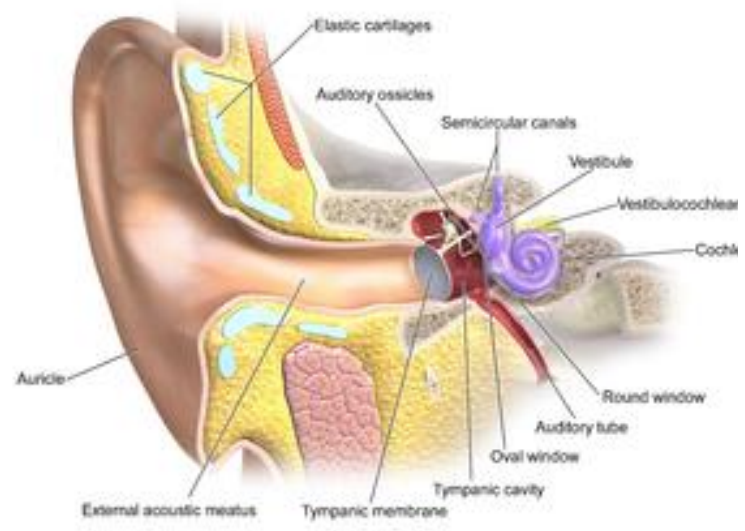

The Anatomy of the Ear

Ear.

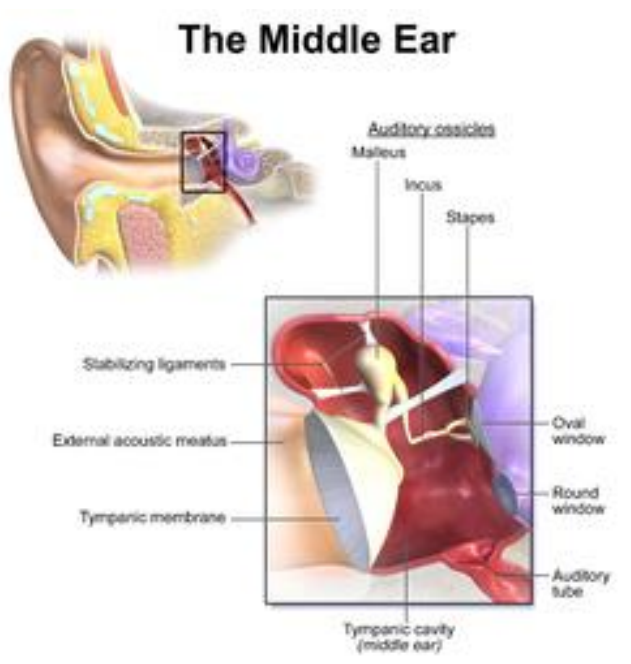

Middle ear.

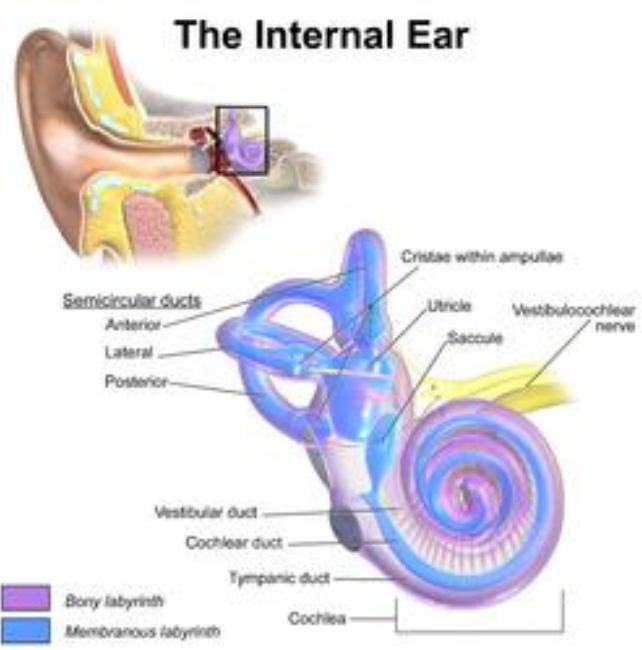

Inner ear. 


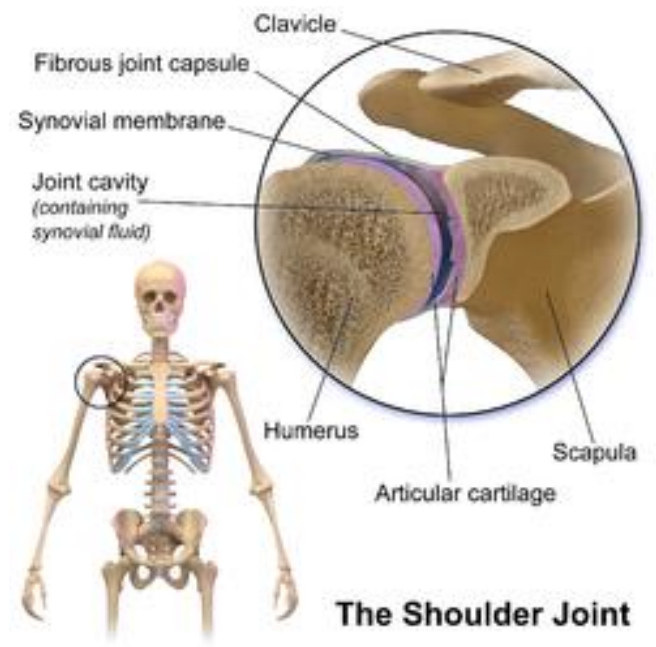

Shoulder joint.

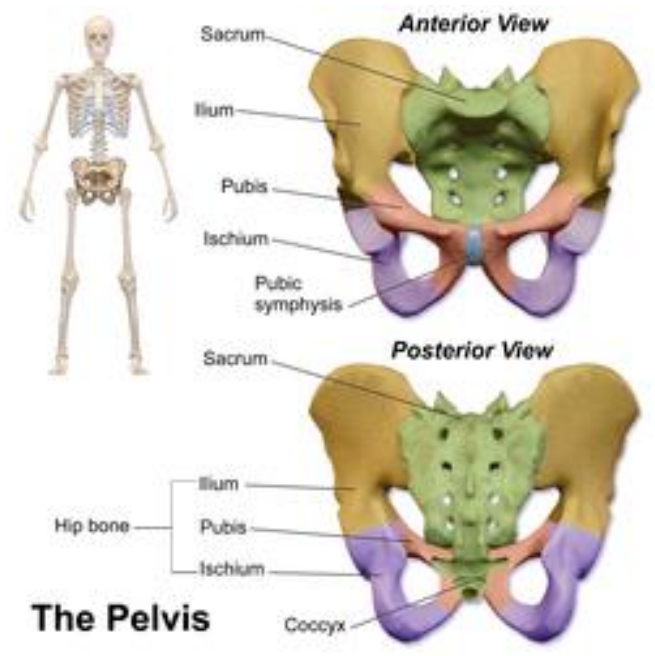

Pelvis.

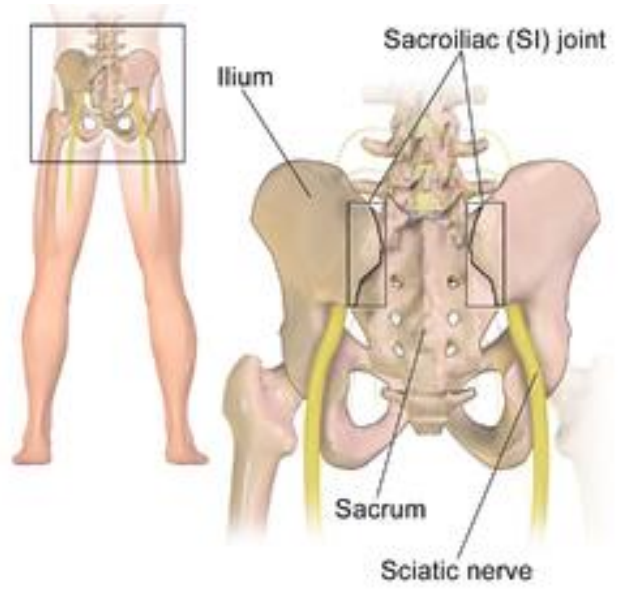

Sacroiliac Joint

Sacroiliac joint.

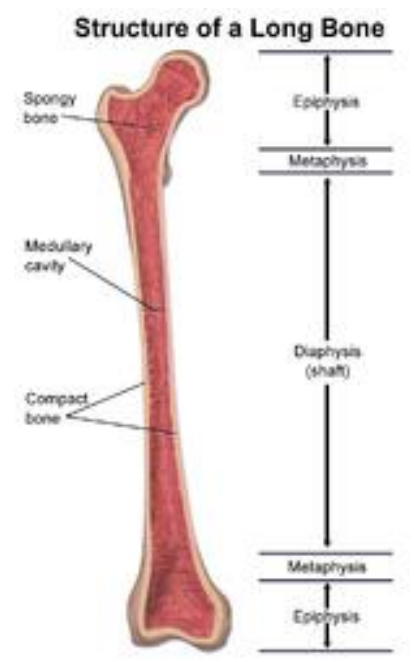

Anatomy of a long bone. 


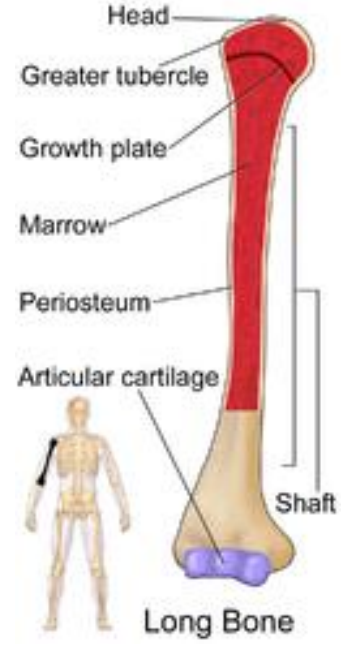

Humerus.

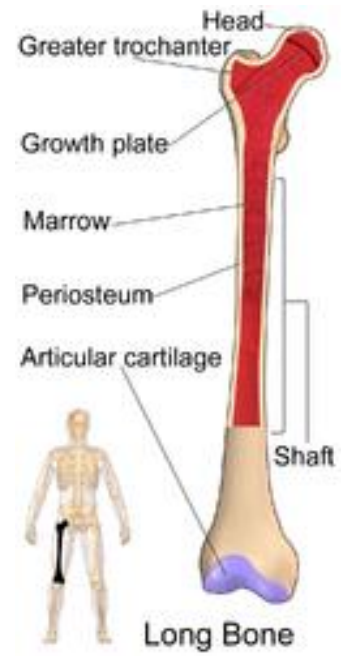

Femur.

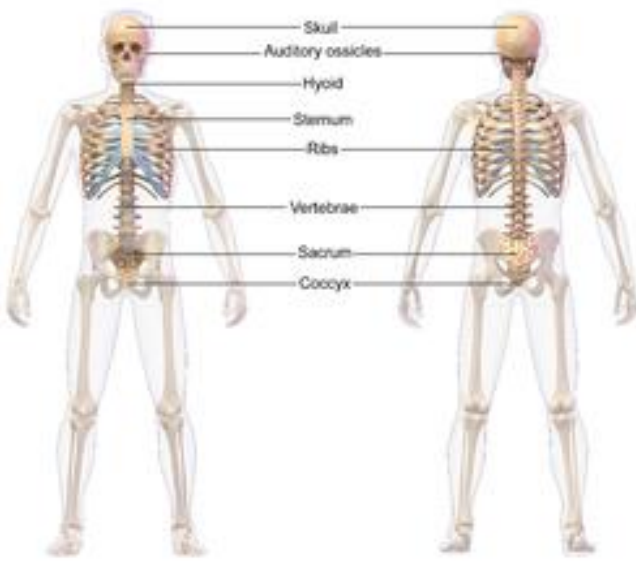

The Axial Skeleton

Axial skeleton.

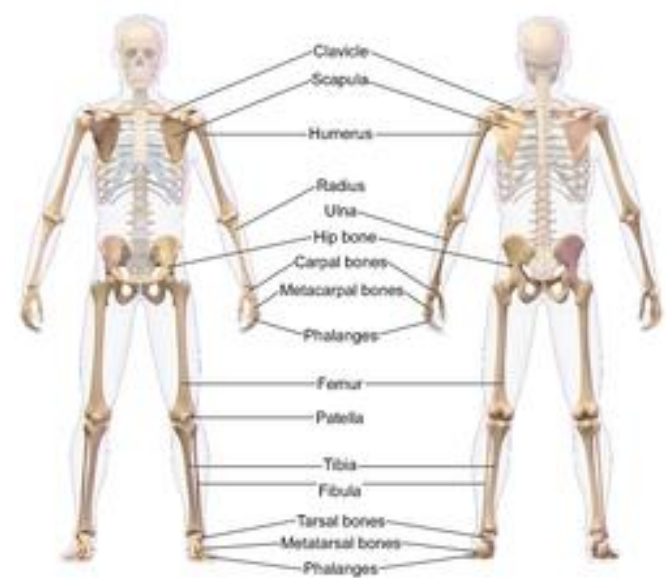

The Appendicular Skeleton

Appendicular skeleton. 


\section{Cell biology}

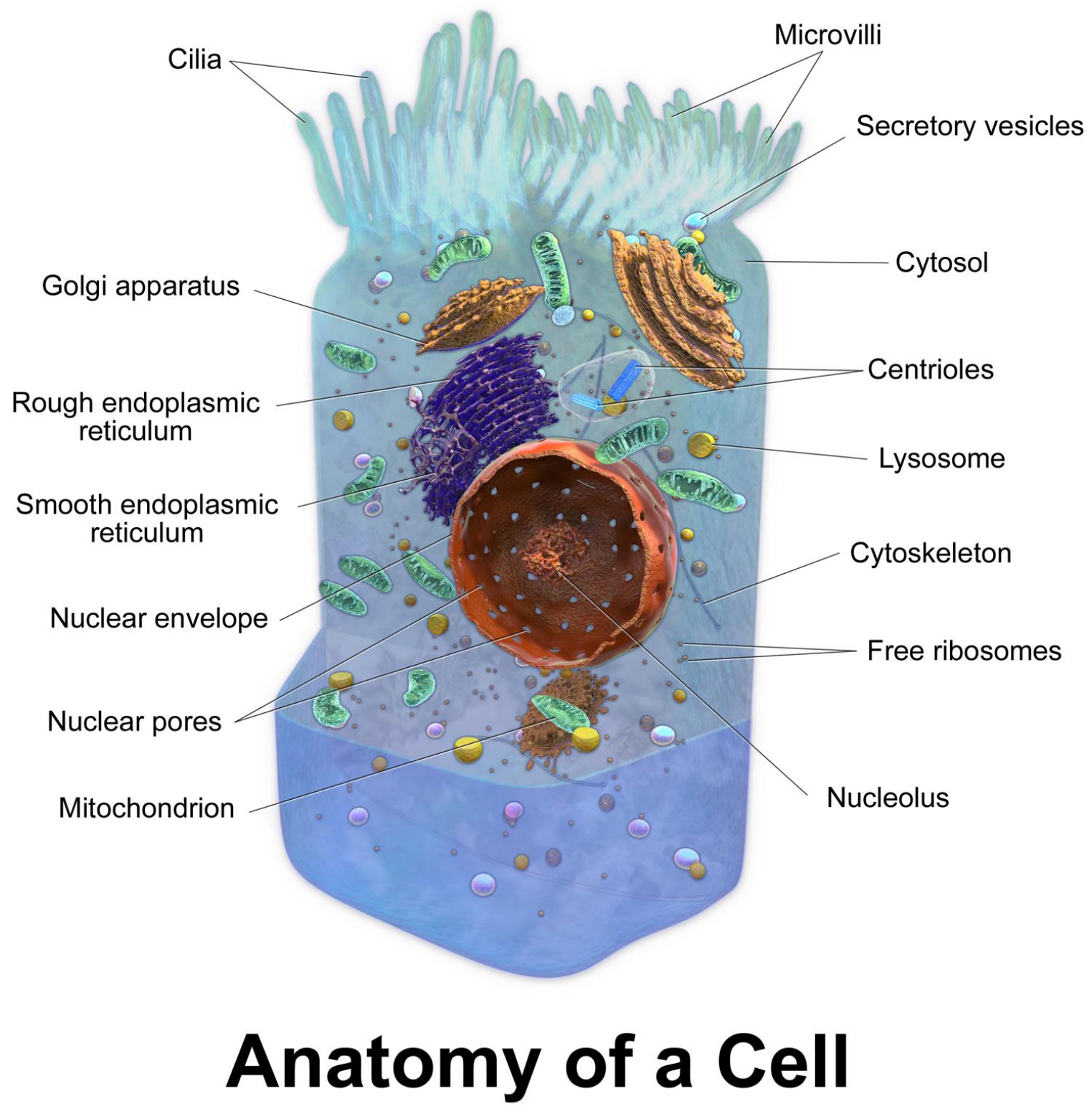

Cell anatomy 


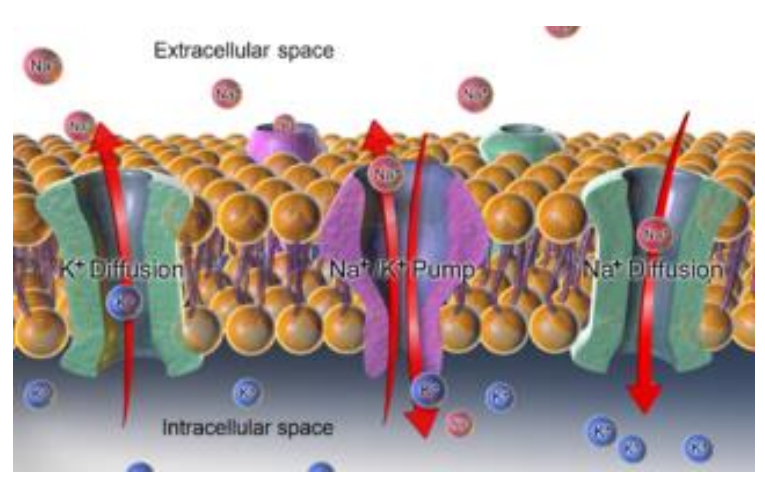

Sodium-potassium pump and resting potential,

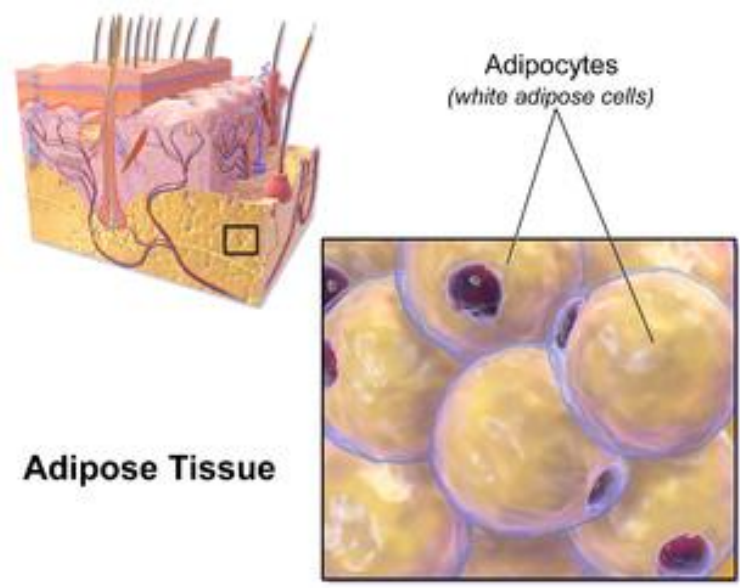

Adipose tissue. 

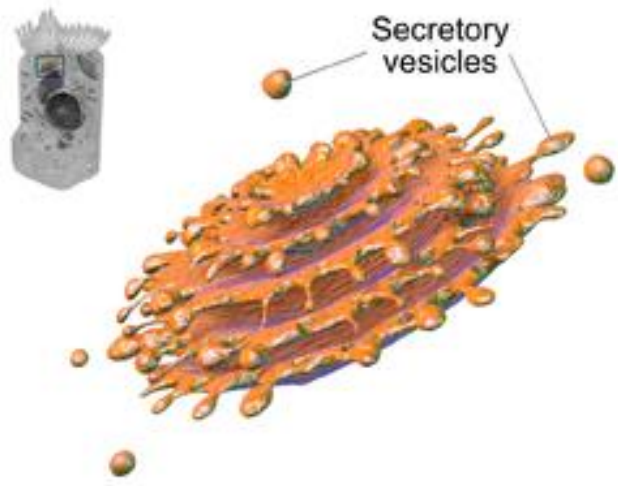

\section{Golgi Apparatus}

Golgi apparatus.

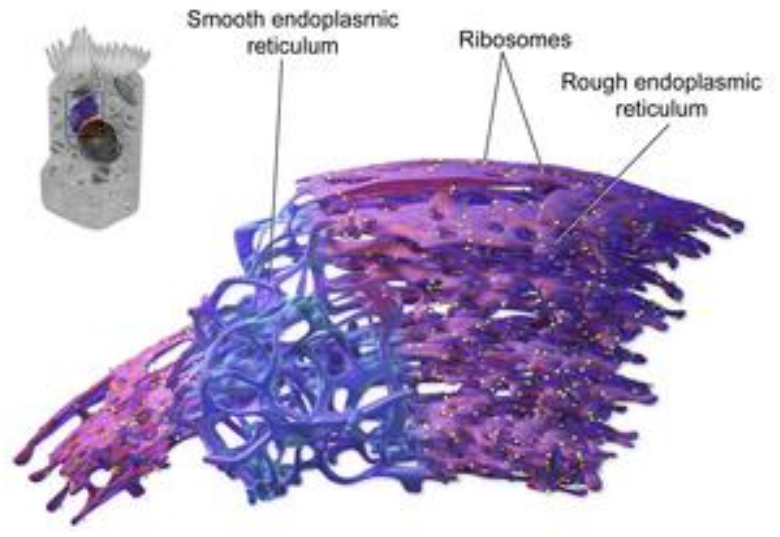

\section{Endoplasmic Reticulum}

Endoplasmic reticulum. 


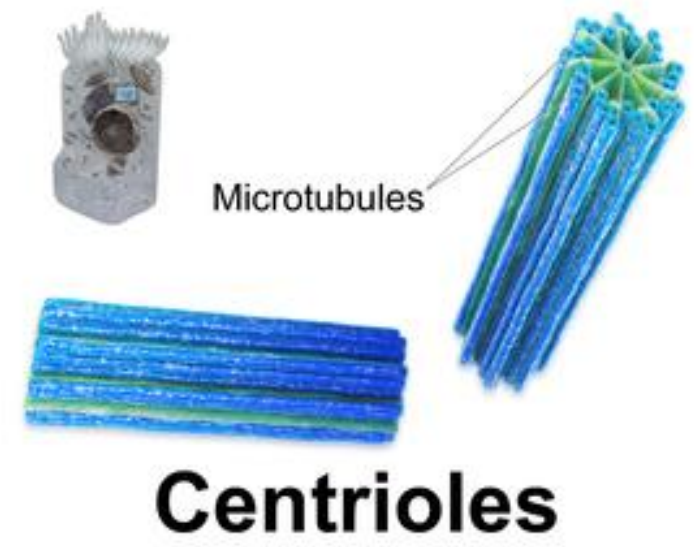

Centrioles.

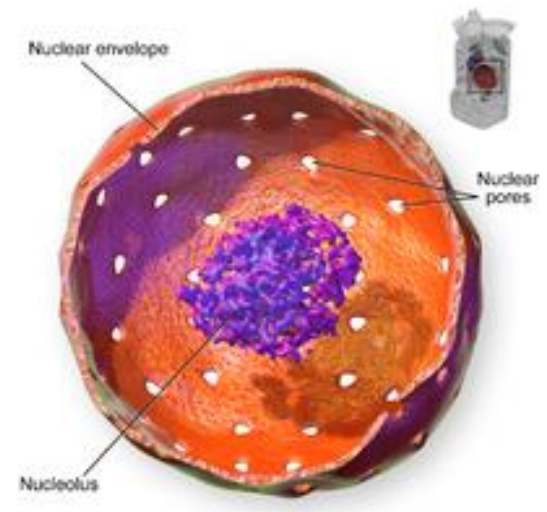

Nucleus

Cell nucleus. 


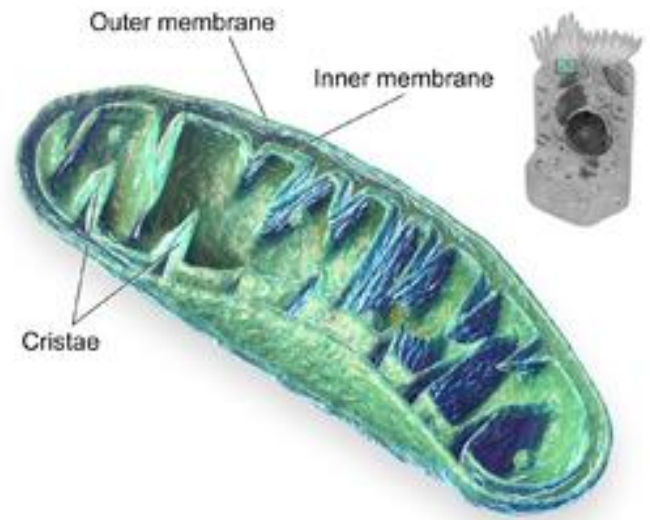

Mitochondria

Mitochondria. 
Biochemistry

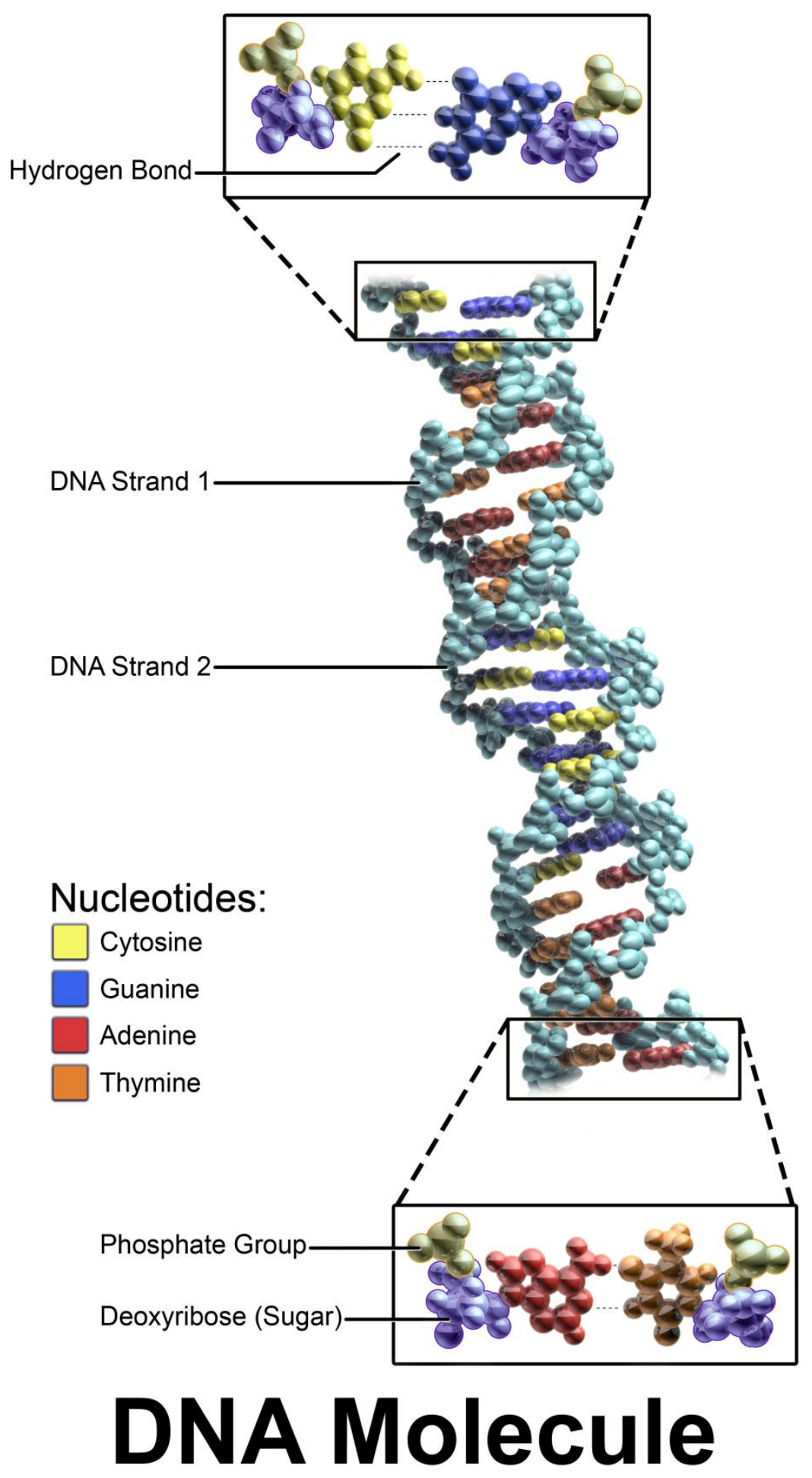

DNA molecule. 


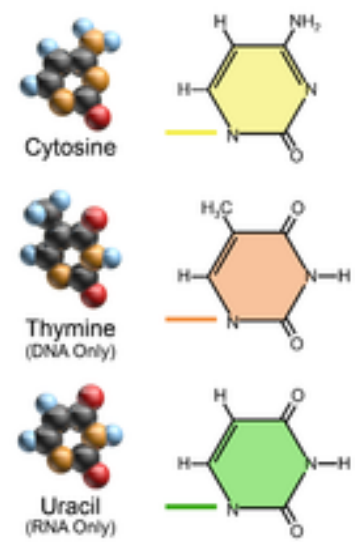

\section{Pyrimidines}

Pyrimidine-derived nucleobases.

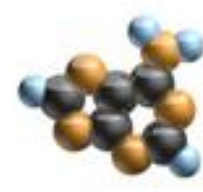

Adenine

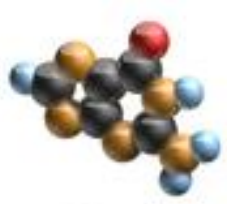

Guanine
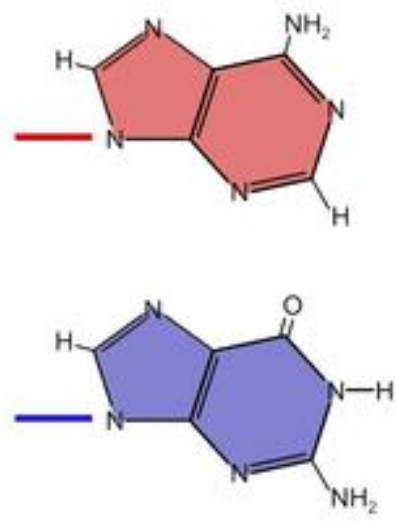

Purines
Purine-derived nucleobases.

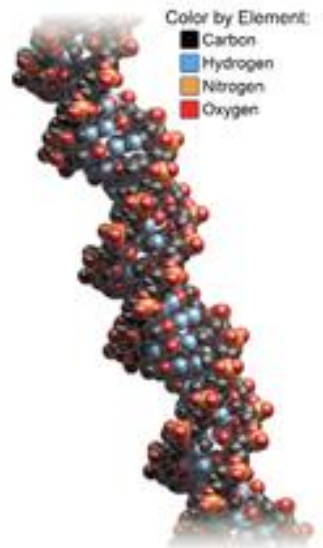

\section{DNA Molecule}

DNA molecule.

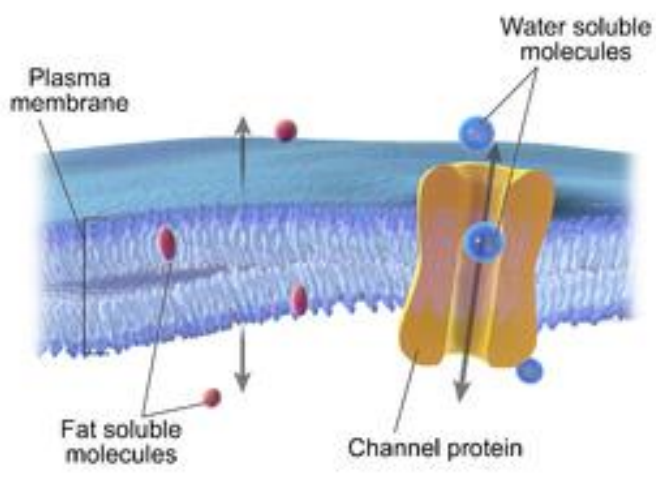

Diffusion Across the Plasma Membrane

Passive transport by diffusion across a cell membrane. 


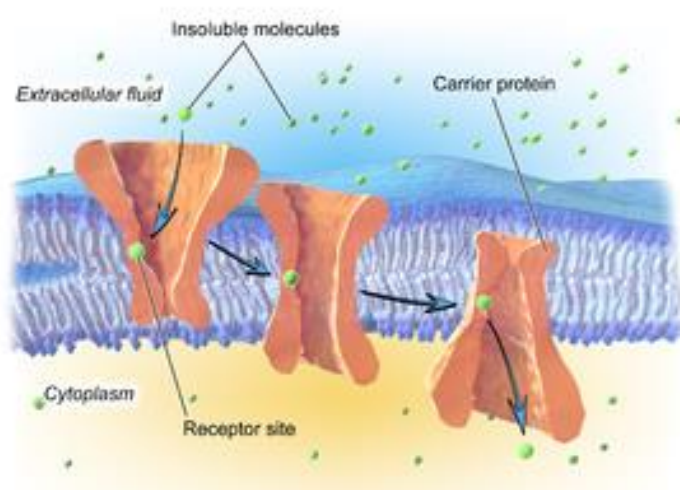

Facilitated Diffusion

Facilitated diffusion.

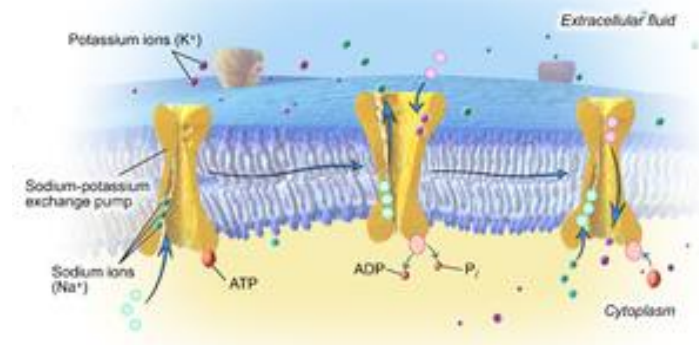

The Sodium-Potassium Exchange Pump

Sodium-potassium pump.

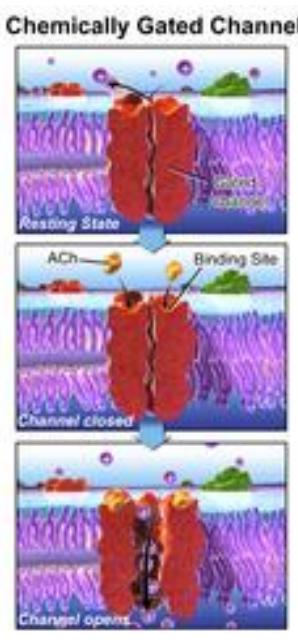

Ligand-gated ion channel.

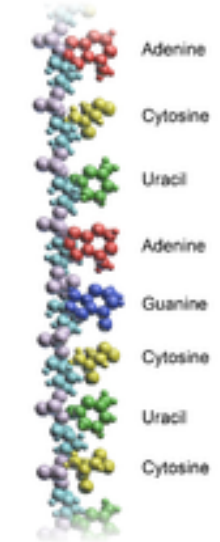

RNA Molecule

RNA molecule. 


\section{Interventions}

Treatments, diagnostic tools and other interventions not already primarily belong to any of the sections above.

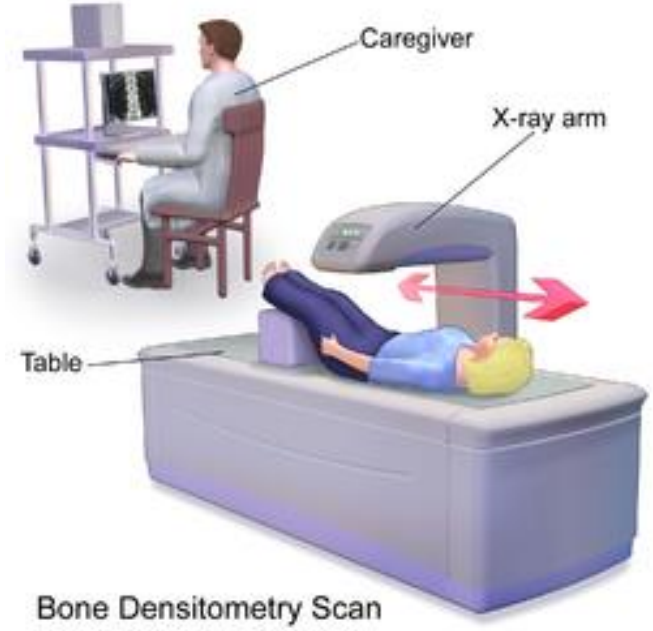

Bone densitometry scan.

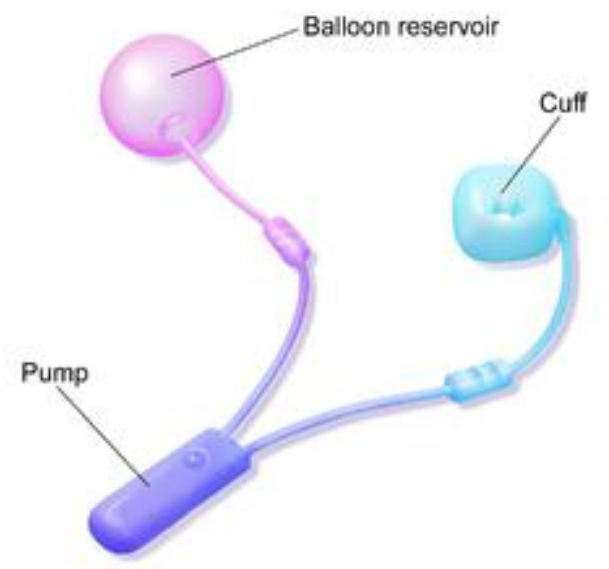

Artificial Urinary Sphincter
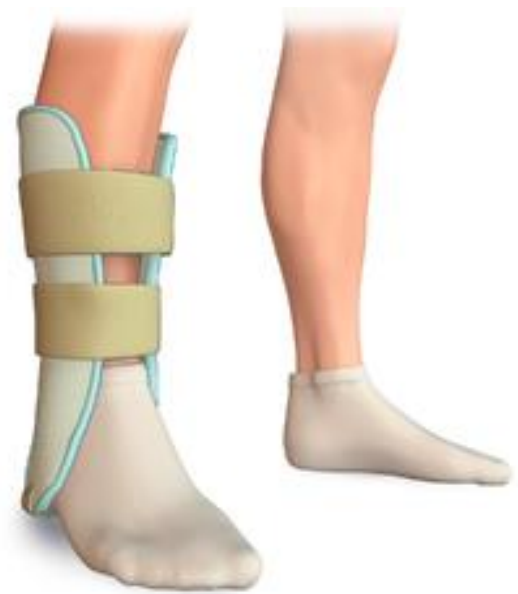

Ankle Stirrup Splint

Ankle stirrup splint.

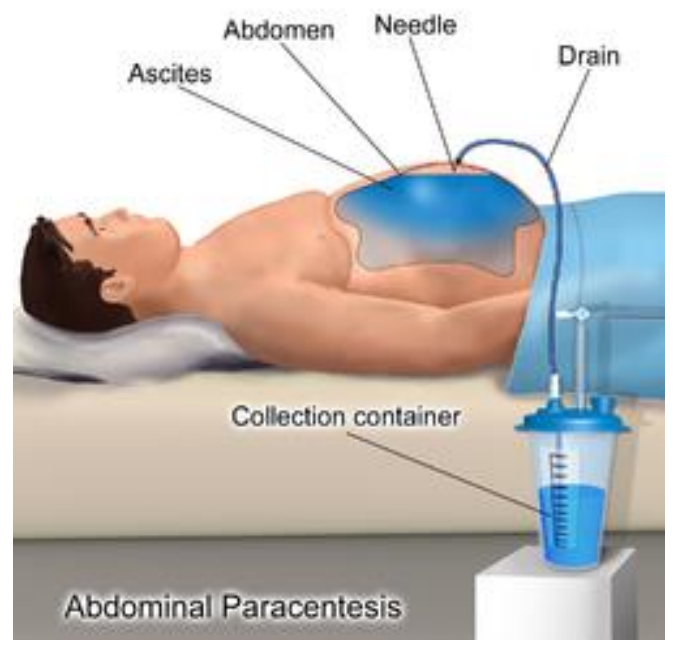

Artificial urinary sphincter.

Abdominal paracentesis. 


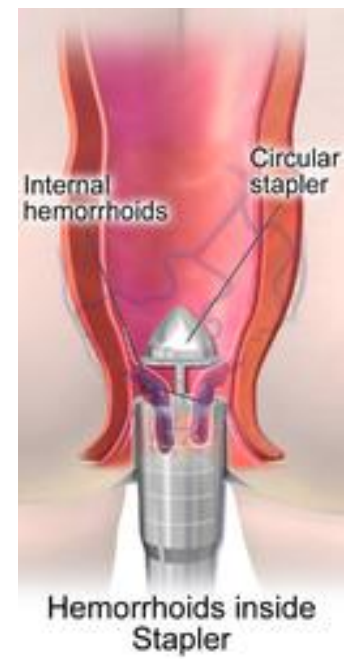

Stapled hemorrhoidopexy.

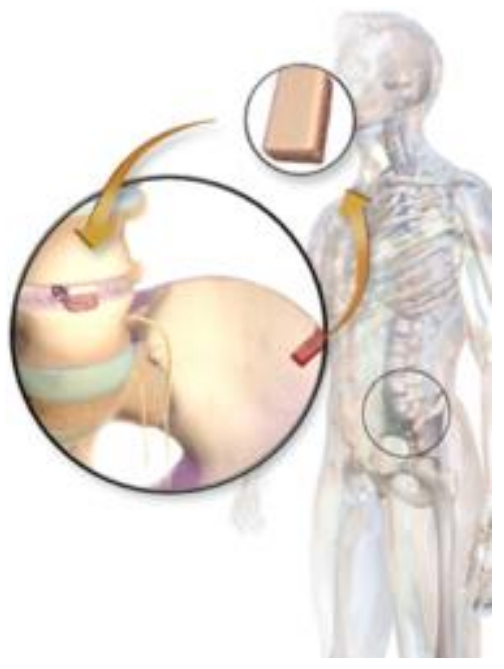

Bone grafting using an autograft.

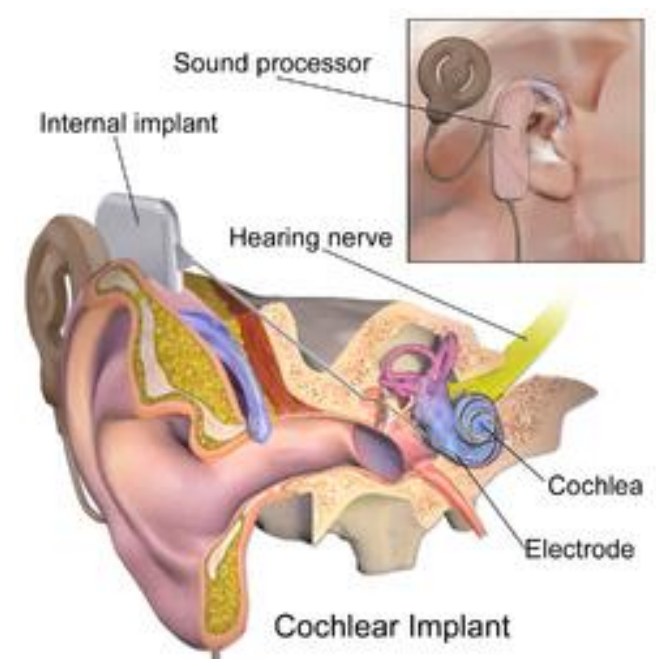

Cochlear implant.

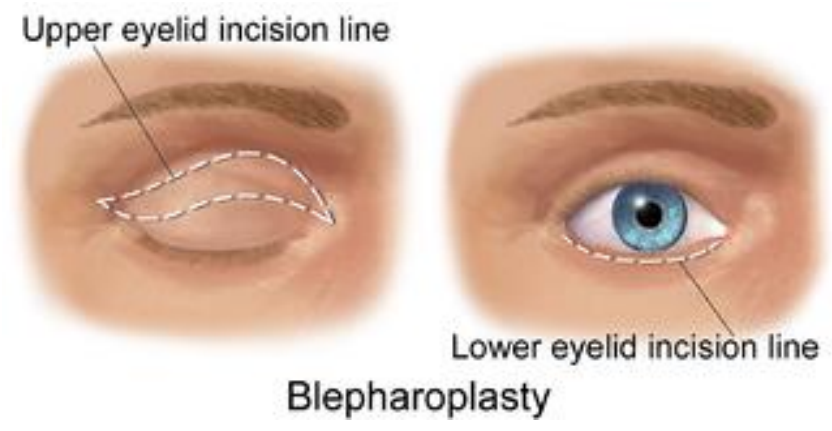

Blepharoplasty.

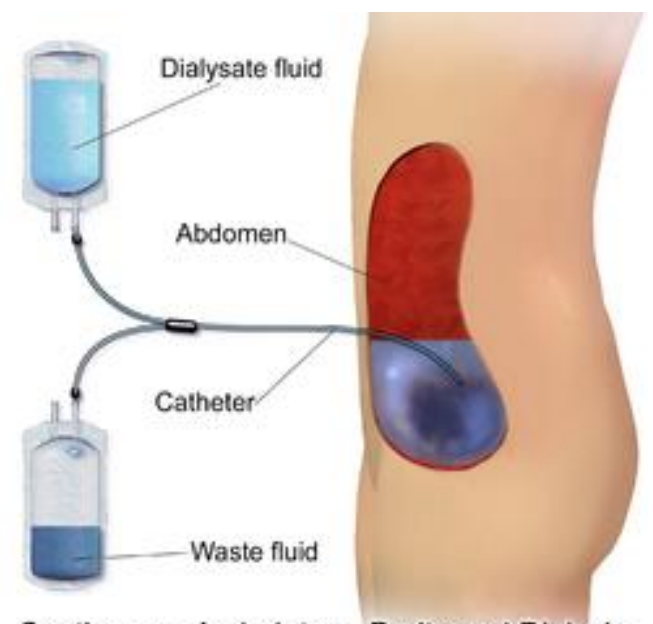

Continuous Ambulatory Peritoneal Dialysis

Continuous ambulatory peritoneal dialysis. 